\title{
Gibbs measures on Brownian currents
}

\author{
Massimiliano Gubinelli \\ Laboratoire de Mathématiques, Université de Paris-Sud \\ Bâtiment 425, F-91405 Orsay Cedex, France \\ massimiliano.gubinelli@math.u-psud.fr \\ József Lőrinczi \\ Zentrum Mathematik, Technische Universität München \\ Boltzmannstr. 3, 85747 Garching bei München, Germany \\ lorinczi@ma.tum.de
}

\begin{abstract}
Motivated by applications to quantum field theory we consider Gibbs measures for which the reference measure is Wiener measure and the interaction is given by a double stochastic integral and a pinning external potential. In order properly to characterize these measures through DLR equations, we are led to lift Wiener measure and other objects to a space of configurations where the basic observables are not only the position of the particle at all times but also the work done by test vector fields. We prove existence and basic properties of such Gibbs measures in the small coupling regime by means of cluster expansion.
\end{abstract}

Keywords: Brownian motion, double Itô integral, stochastic currents, rough paths, Gibbs measure, cluster expansion 


\section{Motivations and outline}

Gibbs measures are familiar objects in various areas of applied probability. Originally they have been devised in the framework of lattice spin systems to describe thermodynamic equilibrium states. Obtaining these probability measures involves two basic steps. First a family of Gibbs measures for a finite number of random variables is constructed as a modification of a reference measure usually describing the independent random field. The density is the exponential of an additive functional dependent on an interaction function. Then one takes a weak limit of these measures by increasing the number of variables to infinity. This procedure copes with the fact that in usual model systems the interaction diverges in this limit, thus the limit measure cannot be directly defined.

The context in which the class of Gibbs probability measures proved to be useful has substantially widened over the years, reaching the realm of Brownian motion. One natural way leading to Gibbs measures on path space is the application of the FeynmanKac formula and its extensions. Suppose $H$ is a self-adjoint operator acting on a Hilbert space $\mathcal{H}$, and let $f \in \mathcal{H}$. Then for a variety of specific choices of $H$ an equality of the type

$$
\left(e^{-t H} f\right)(x)=\int_{\mathbb{R}^{d}} d y \int f\left(X_{t}\right) d \mu_{[0, t]}(X \mid x, y)
$$

can be derived, where $\mathbb{R} \ni t \mapsto X_{t} \in \mathbb{R}^{d}$ is Brownian motion and

$$
d \mu_{[0, t]}(X \mid x, y)=e^{-U_{t}(X \mid x, y)} d \mathcal{W}_{[0, t]}^{x, y}(X) .
$$

Here $\mathcal{W}_{[0, t]}^{x, y}$ is Brownian bridge over the bounded time interval $[0, t]$, starting at $X_{0}=x$ and ending at $X_{t}=y$, and $U_{t}$ is a functional of Brownian paths derived from $H$. After normalizing to 1 , the measure $\mu_{[0, t]}(X \mid x, y)$ can be viewn as a Gibbs measure on path space for "finite volume" $[0, t]$, "interaction" $U$, "boundary condition" $x, y$, and "reference measure" $\mathcal{W}^{x, y}$.

In this paper we are interested in the question whether an extension in a suitable sense over the full time line $\mathbb{R}$ of $\mu_{[0, t]}$ exists. The details of the proof strongly depend on the choice of $U$, which for our purposes here will be specified below. These choices are motivated by particular applications covered by the following classes of models, of which we talk only in this introduction.

I. Densities dependent on the local time of Brownian motion

(1) $P(\phi)_{1}$-process (Itô diffusion) This includes the familiar case of Schrödinger operators $H=(-1 / 2) \Delta+V, \mathcal{H}=C_{0}^{\infty}\left(\mathbb{R}^{d}\right)$, with a potential $V(x)$ that can be chosen fairly generally (Kato-class). The well-known result is

$$
U_{t}(X)=\int_{0}^{t} V\left(X_{s}\right) d s .
$$

$\mu$ describes in this case the path measure of the Markov process given by

$$
d X_{t}=d B_{t}+(\nabla \log \Psi)\left(X_{t}\right) d t
$$


where $B_{t}$ is $\mathbb{R}^{d}$-valued Brownian motion and $\Psi$ is the eigenfunction of $H$ lying at the bottom of its spectrum (ground state). Itô diffusions can be defined also on infinite dimensional spaces related to other SPDE. For further details on these Gibbs measures for bounded intervals and their extensions to $\mathbb{R}$ we refer to [46, 47, 4].

(2) Nelson's model This is a scalar quantum field model describing the interaction of an electrically charged spinless particle with a boson field. In this case $H$ is written as the sum of the free particle Hamiltonian $(-1 / 2) \Delta+V$, the free field Hamiltonian $\int|k| a(k)^{*} a(k) d k$ with the usual boson creation and annihilation operators $a^{*}$ and $a$, and the interaction Hamiltonian $\int(\widehat{\rho}(k) / \sqrt{2|k|})\left(e^{i k \cdot x} a(k)+e^{-i k \cdot x} a^{*}(k)\right) d k$, with charge distribution function $\rho$. Moreover, $\mathcal{H}=L^{2}\left(\mathbb{R}^{d}, d x\right) \otimes \mathcal{F}$, where $\mathcal{F}$ is Fock space, and a Feynman-Kac-type formula as (1.1) above can be obtained by mapping $\mathcal{H}$ into a space of continuous functions through a joint use of the so called ground state transform and Wiener-Itô isomorphism yielding

$$
U_{t}(X)=\int_{0}^{t} V\left(X_{s}\right) d s+\int_{0}^{t} \int_{0}^{t} W^{\rho}\left(X_{s}-X_{r}, s-r\right) d s d r
$$

with

$$
W^{\rho}(x, s)=-\frac{1}{4} \int_{\mathbb{R}^{d}} \frac{|\widehat{\rho}(k)|^{2}}{|k|} e^{-i k \cdot x-|k||s|} d k .
$$

Extending this Gibbs measure from $[0, t]$ to $\mathbb{R}$ is in this case of special interest since it allows a direct expression of the ground state of $H$ in terms of its Radon-Nikodym derivative with respect to an underlying product measure, which makes a rigorous derivation and proof of ground state properties possible. For the case of translation invariant models (involving $V \equiv 0$ ) there are only few results available. For details see [37, 38, 39, 3.

(3) Polaron and bipolaron models The polaron is a "dressed" electron (i.e., embedded into an energy cloud) interacting with a phonon field (i.e., quantum particles carrying the vibrational energy of an ionic crystal). In this case we have similar operators acting on the same Hilbert space as above except that the dispersion relation $|k|$ is replaced by 1 in the free field Hamiltonian, and $\widehat{\rho} / \sqrt{2|k|}$ in the interaction term by $1 /|k|$. This leads to the same $U_{t}$ as in the case of Nelson's model with

$$
W^{\mathrm{pol}}(x, s)=-\frac{1}{4|x|} e^{-|s|} .
$$

The bipolaron differs by the fact that it consists of two dressed electrons coupled to the same phonon field, which are repelling each other by Coulomb interaction. In this case

$$
U_{t}(X)=\alpha^{2} \int_{0}^{t} \int_{0}^{t} \mathcal{E}^{W}\left(X_{s}, Y_{r}, s-r\right) d s d r-g \int_{0}^{t} \frac{d s}{\left|X_{s}-Y_{s}\right|}
$$

where

$$
\mathcal{E}^{W}\left(X_{s}, Y_{r}, u\right)=W\left(X_{s}-X_{r}, u\right)+2 W\left(X_{s}-Y_{r}, u\right)+W\left(Y_{s}-Y_{r}, u\right)
$$


with $W=W^{\text {pol }}, \alpha<0$ being the polaron-phonon coupling paramater and $g>0$ the strength of the Coulomb repulsion between the two polarons. Moreover, in this case the reference measure is a product of two independent Wiener measures. For literature see [14, 45, 36].

(4) Intersection local time (weakly self-avoiding polymer) Formally, the densities are given by

$$
U_{t}(X)=\int_{0}^{t} \int_{0}^{t} \delta\left(X_{s}-X_{r}\right) d s d r
$$

meant to describe a polymer model with short-range "soft-core" interaction encouraging to avoid self-intersections. For $d=2,3$ see [49, [51, 50, 52, 5]. In [48] it was proved that in $d=2$ the model can be rigorously defined after an additive renormalization and the so obtained measure is absolutely continuous with respect to Wiener measure in 2 dimensions. In $d=3$ the singularity of the energy $U_{t}$ is more serious but an additive renormalization still suffices; Westwater proved existence of the Gibbs measure which, however, in this case is not absolutely continuous with respect to Wiener measure. Other works include [6, 31, 30, 53].

II. Stochastic currents

(1) Nelson's model in point charge limit and Pauli-Fierz model The point charge limit of Nelson's model corresponds to the case of replacing $\rho$ above with a delta-function, while the Pauli-Fierz model is obtained by replacing the scalar boson field with a quantized Maxwell (vector) field. The function in the density in both cases formally becomes

$$
U_{t}(X)=\int_{0}^{t} V\left(X_{s}\right) d s+\int_{0}^{t} \int_{0}^{t} W\left(X_{s}-X_{r}, s-r\right) d X_{s} \cdot d X_{r},
$$

with a $W=W^{\delta}$ and $W=W^{\mathrm{PF}}$ we do not write explicitly down here. The difference from the case above is that instead of double Riemann we have to deal with double Itô integrals. A substantial difficulty to solve in this case is the proper definition of the double integrals in the first place, which will be done below. For the specific model applications see [24] in which we perform an ultraviolet renormalization of Nelson's Hamiltonian by using path measures whose densities are of the above form, and [26, 28, for the Pauli-Fierz model. The similar point charge limit in the latter model is an open problem.

(2) Turbulent fluids In fluid dynamics the understanding prevails that fully developed turbulence should be described by a suitable measure over divergence-free velocity fields $u(x)$. One way of modelling it starts from the assumption that the vorticity field $\nabla \wedge u(x)$ is concentrated along Brownian curves $X_{t} \in \mathbb{R}^{3}$. Under the Eulerian incompressible flow, the kinetic energy $(1 / 2) \int u(x)^{2} d x$ is conserved. The formal expression of the total energy is

$$
U_{t}(X)=\int_{0}^{t} \int_{0}^{t} \frac{1}{\left|X_{t}-X_{s}\right|} d X_{t} \cdot d X_{s}
$$


In order to have $e^{-U_{t}(X)}$ as a well-defined random variable at all, 16 imposed the condition that the Coulomb potential is mollified so that the fluid has finite static energy. For details we refer to [20, 18, 42, 1, 19].

III. Processes with jumps

Applications include Hamiltonians with spin. Since spin is a discrete variable, this case goes beyond stochastic integration with respect to Brownian motion alone. The spin paths $\sigma_{t}$ are described via a Poisson process $N_{t}$, and we obtain

$$
U_{t}(X)=\int_{0}^{t} V\left(X_{s}\right) d s+\int_{0}^{t} a\left(X_{t}\right) \circ d X_{t}-\int_{0}^{t} S\left(X_{t}, \sigma_{t}\right) d t+\int_{0}^{t^{+}} \Phi\left(X_{t},-\sigma_{t}\right) d N_{t} .
$$

For more details and explicit formulas of the terms above see [10, 27].

In this paper we address the purely mathematical problem of existence and characterization of Gibbs measures with densities of the type (1.3). An accompanying paper 24] takes this further to an application to quantum field theory.

The problem can be formulated in more generality by adding a term to $U_{t}$ in (1.3) taking into account the dependence on outside paths of paths run within bounded time intervals. Choose without loss a bounded interval in the form $[-T, T] \subset \mathbb{R}$ and set up Wiener measure $\mathcal{W}_{T}$ on it. Consider the energy functions corresponding to $[-T, T]$ as follows:

$$
\begin{aligned}
& V_{T}(X):=\int_{-T}^{T} V\left(X_{s}\right) d s \quad \text { for external potential } V \\
& W_{T}(X):=\int_{-T}^{T} \int_{-T}^{T} W\left(X_{t}-X_{s}, t-s\right) d X_{t} \cdot d X_{s} \quad \text { internal energy } \\
& W_{T}(X \mid Y):=W_{T}^{+}(X \mid Y)+W_{T}^{-}(X \mid Y) \quad \text { interaction energy }
\end{aligned}
$$

where the interaction energies come from a pair interaction potential $W$, and $W_{T}^{+}(X \mid Y):=$ $\int_{T}^{\infty} d Y_{t} \int_{-T}^{T} d X_{s} W\left(X_{s}-Y_{t}, t-s\right)$ is a term accounting for the interaction of paths inside $[-T, T]$ with paths in $[T, \infty)$, and $W_{T}^{-}(X \mid Y):=\int_{-\infty}^{-T} d Y_{t} \int_{-T}^{T} d X_{s} W\left(X_{s}-Y_{t}, t-s\right)$ for external paths running in $(-\infty,-T]$. As in (1.2), these energies give rise to the Gibbs measure for $[-T, T]$

$$
d \mu_{T}(X):=\frac{e^{-V_{T}(X)-\lambda W_{T}(X)}}{Z_{T}} d \mathcal{W}_{T}(X)
$$

with a parameter $\lambda \in \mathbb{R}$ tuning the strength of the pair potential, and $Z_{T}$ the normalizing factor turning it into a probability measure. The object of interest are the accumulation points $\mu$ of the family of measures $\left\{\mu_{T}\right\}_{T>0}$ in the topology of local weak convergence. In the DLR (Dobrushin-Lanford-Ruelle) approach, these limit points can be characterized by a property of consistency with respect to a prescribed family of probability kernels (specification) providing the local conditional probabilities of the limit random fields. These kernels are given by

$$
\mu_{T}(d X \mid Y):=\frac{e^{-V_{T}(X)-\lambda W_{T}(X)-\lambda W_{T}(X \mid Y)}}{Z_{T}(Y)} d \mathcal{W}_{T}^{Y_{-T}, Y_{T}}(X)
$$


with external (or boundary) path $Y$. The possible accumulation points of the family $\left\{\mu_{T}\right\}_{T}$ should then satisfy the DLR equations $\int \mu_{T}(A \mid Y) \mu(d Y)=\mu(A)$ for all cylinder sets $A$ in the sub- $\sigma$-field generated by projections to $[-T, T]$ for all $T>0$.

In contrast to the case of interactions depending on the local time of the process $X$ (given by double Riemann integrals listed above) our case encounters two difficulties.

(1) The expressions of $W_{T}(X)$ and $W_{T}(X \mid Y)$ are only formal: The double stochastic integrals are not well defined since the integrands are neither forward nor backward adapted with respect to the semimartingale $X$ (Brownian bridge under $\mathcal{W}_{T}^{x, y}$ ).

(2) The specification (1.6) must be defined pathwise for each $Y$, however, in general the only information we have on the boundary path $Y$ is that it is a continuous path with a Brownian-like regularity. This is insufficient for defining the line integrals with respect to $d Y$ appearing in $W_{T}(X \mid Y)$.

Of these two difficulties the first requires a minor amendment, while the second is far more serious and will urge us to introduce the novel setting of Brownian currents in which we can make sense of a formulation of the DLR equations. Roughly speaking, we provide each sample path with sufficient information in order to determine the work made by test vector fields. Specifically, we consider the family of random variables $\left\{X_{t}\right\}_{t}$ jointly with the random variables $C_{s t}^{X}(\varphi):=\int_{s}^{t} \varphi\left(u, X_{u}\right) d X_{u}$ (stochastic currents) and show that in this augmented sample space the specification can pathwise be defined. (This problem has some analogy with difficulties at defining specifications for unbounded spin systems for which too one has to select a subset of admissible boundary conditions so that the interaction energy makes sense. Our approach here goes further in that we do not only have to control the growth of the boundary paths but must also provide a priori the value of the line integrals against a sufficiently large set of test vector fields $\varphi$.) To perform this "lifting" procedure we use techniques of rough paths theory.

Here is an outline of the paper. In Chapter 2 we present our results on rough paths that will be put at use subsequently. In Chapter 3 we introduce the framework of stochastic currents, in particular Brownian currents. In Chapter 4 we define Gibbs measures on these currents for bounded intervals of the line. In Chapter 5 we turn to proving that these Gibbs measures can be extended over the whole line provided the interaction term is weakly coupled. This requirement is needed since, faute de mieux, we use cluster expansion in order to construct weak limits of Gibbs measures for bounded intervals. The version of cluster expansion we develop here is different from the conventional ones since the configurations are segments of Brownian paths rather than spins of compact or real-valued state space, the interactions depend on double stochastic integrals, the reference measure is not a product measure, and the measure we want to construct is non-Markovian. However, it has the same spirit of usual cluster expansions as it splits off into a part of hands-on analysis of energy bounds and a part of combinatorics. Beside a proof of existence, cluster expansion allows us to study also uniqueness, typical path behaviour and mixing properties of Gibbs measures, which will be done in Chapter 6 . 


\section{Rough paths}

\subsection{Definition of double stochastic integrals by rough paths}

Itô's theory of integration gives a meaning to line integrals of the form $\int_{-T}^{T} \varphi\left(t, X_{t}\right) d X_{t}$, with $X$ a semimartingale (with respect to its natural forward filtration $\mathcal{F}$ ) and $\varphi(t, \xi)$ some function. The way of obtaining it is by taking limits of Riemann sums $\sum_{\alpha} \varphi\left(\tau_{\alpha}, X_{\tau_{\alpha}}\right)\left(X_{\tau_{\alpha+1}}-\right.$ $X_{\tau_{\alpha}}$ ) over a family of partitions $\left\{\tau_{\alpha}\right\}_{\alpha}$ of $[-T, T]$ with mesh decreasing to zero. Such limits are known to exist whenever $\varphi\left(t, X_{t}\right)$ is an $\mathcal{F}$-adapted functional of $X$, i.e., if it only depends on $\mathcal{F}_{t}$ for any $t \in[-T, T]$ and has some integrability properties. Then $\int_{-T}^{T} \varphi\left(t, X_{t}\right) d X_{t}$ is defined as a random variable on the same probability space on which $X$ is defined. In our applications $X$ is the coordinate process on the Borel space $\mathcal{X}$, and the stochastic integral defines a Borel map from $\mathcal{X}$ to $\mathbb{R}$ which is defined on a set of full $\mathcal{W}$ measure. It is important to remember the crucial fact that the full-measure set in general depends on the integrand: different integrands may give different full-measure sets.

In this section we analyze the regularity of such integrals by using the theory or rough paths as developed in [33, 34, 23, 15]. This theory allows to properly understand stochastic integrals from a purely analytic perspective. To gain this freedom we need to enlarge the sample space over which the measures are defined, however, this will turn out to make no harm in our applications.

Rough paths theory has been devised by T. Lyons [33] to give a meaning to line integrals of the form

$$
\int_{0}^{T} \varphi\left(X_{t}\right) d X_{t}
$$

in case of $X$ being an irregular function of the parameter. A typical case of interest is when $X$ is chosen to be Hölder continuous with small exponent, such as $\gamma \in(0,1)$. The natural approach to defining such integrals is that of taking Riemann approximations over a finite partition of $[0, T]$ and proving that the sequence converges as the mesh of the partition goes to zero. When general integrals in the form $\int_{0}^{T} Y_{s} d X_{s}$ are considered with bounded $Y$, this can only hold if $X$ is a process of bounded variation leading to the familiar Stieltjes integral. When $Y$ is Hölder continuous with exponent $\rho$, Young's work [54 makes sure that a sufficient condition for the convergence of the Riemann sums is that $\gamma+\rho>1$. The Young integral is useful in studying, for example, fractional Brownian motion of Hurst index $H>1 / 2$, which is a stochastic process with Hölder continuous paths of any exponent $\gamma<H$. In this case (2.1) can be defined for any function $\varphi$ which is at least $C^{1}$.

Integrals of the form (2.1) when $X$ is a sample of Brownian motion are not within the reach of Young's theory and indeed it is not difficult to see that different definitions of the Riemann approximations can lead to different limits (or even not converge at all). This difficulty lies at the basis of the existence of more than one type of stochastic integral over Brownian motion, two well known possibilities being the integrals developed by Itô and Stratonovich. Lyons's approach to the problem was proving that under reasonable conditions the Riemann sums for $\int_{0}^{T} \varphi\left(X_{t}\right) d X_{t}$ can be modified by adding an extra term in order to make them converge. This compensation is not unique but in many relevant 
cases it can be performed in such a way that the so obtained integral is an extension of the classical line integral and Young integral. That is, whenever $X$ is regular enough, the modified integral coincides with the Young one or Riemann-Lebesgue integral if moreover $X$ is almost surely is differentiable.

We consider only the case when $X$ is Hölder continuous with $\gamma>1 / 3$ since this includes the case of Brownian motion and all our present applications. (The theory for more general $\gamma$ would be far more cumbersome.) We use the notation $X_{s t}=X_{t}-X_{s}$, and make the following basic

Definition 2.1 For each bounded $I \subset \mathbb{R}$ we call the couple $(X, \mathbb{X})$ a 2-step rough path, where

(1) $X \in C^{\gamma}\left(I, \mathbb{R}^{d}\right)$;

(2) there exists a function $\mathbb{X}: I \times I \rightarrow \mathbb{R}^{d} \times \mathbb{R}^{d}$ satisfying the multiplicative property

$$
\mathbb{X}_{s t}^{i j}-\mathbb{X}_{s u}^{i j}-\mathbb{X}_{u t}^{i j}=X_{s u}^{i} X_{u t}^{j}
$$

for all $s \leq u \leq t \in I$ and any $i, j=1, \ldots, d$;

(3) there is $C \in[0, \infty)$ such that

$$
\left\|\mathbb{X}_{s t}\right\|_{2 \gamma}:=\sup _{t \neq s} \frac{\left|\mathbb{X}_{s t}\right|}{|t-s|^{2 \gamma}} \leq C
$$

We use the similar norm $\left\|X_{s t}\right\|_{\gamma}$ correspondingly given by the right hand side of (2.3).

Remark 2.2 Note that Assumption (2) is non-trivial only if $d>1$. When $d=1$ we can take $\mathbb{X}_{s t}=\left(X_{s t}\right)^{2} / 2$, and it can easily be seen that both (2.2) and the Hölder-like bound (2.3) are then satisfied.

For our purposes below we need a slight extension since we want to be able to integrate functions explicitly dependent on the time parameter, such as $\int_{0}^{T} \varphi\left(u, X_{u}\right) d X_{u}$. One possibility is to consider the couple $(u, X)$ as a new path and construct the associated rough path. This construction, however, has the disadvantage of requiring rather much regularity of the function $\varphi$. A more effective approach is to treat the line-integral as a Young integral with respect to the $u$ dependence of the integrand as soon as the map $u \mapsto \varphi(u, \xi)$ has Hölder exponent greater than $1-\gamma$ (as we shall see below).

For the (time-dependent) test vector fields in $C\left(\mathbb{R} \times \mathbb{R}^{d} ; \mathbb{R}^{d}\right)$ we use the norm

$$
\|\varphi\|_{\rho, 2, s, t}=\sup _{x}\left[\sup _{u \in[s, t]}\left(\max _{k=0,1,2}\left|\nabla^{k} \varphi(u, x)\right|\right)+\sup _{u, v \in[s, t]} \max _{k=0,1} \frac{\left|\nabla^{k} \varphi(u, x)-\nabla^{k} \varphi(v, x)\right|}{|u-v|^{\rho}}\right]
$$

with $\rho>0$ and the convention $\nabla^{0} \varphi(t, x)=\varphi(t, x)$. Then our basic result on the step-2 rough path $(X, \mathbb{X})$ is 
Theorem 2.3 Let $\varphi \in C\left(\mathbb{R} \times \mathbb{R}^{d}, \mathbb{R}^{d}\right)$ be such that it is $C^{2}$ with respect to its second variable and Hölder continuous with exponent $\rho$ with respect to its first variable, such that $\rho+\gamma>1$. Then the sums

$$
\sum_{\alpha}\left(\varphi_{i}\left(\tau_{\alpha}, X_{\tau_{\alpha}}\right) X_{\tau_{\alpha+1} \tau_{\alpha}}^{i}+\nabla_{j} \varphi_{i}\left(\tau_{\alpha}, X_{\tau_{\alpha}}\right) \mathbb{X}_{\tau_{\alpha} \tau_{\alpha+1}}^{i j}\right), \quad i, j=1, \ldots, d
$$

converge as the mesh of the partition $\left\{\tau_{\alpha}\right\}_{\alpha}$ of $[0, T]$ goes to zero, and defines the integral $\int_{0}^{T} \varphi\left(u, X_{u}\right) d X_{u}$ Moreover for any $T \leq 1$ we have the bound

$$
\left|\int_{0}^{T} \varphi\left(u, X_{u}\right) d X_{u}\right| \leq C T^{\gamma}\|\varphi\|_{\rho, 2,0, T}\left(1+\|X\|_{\gamma}+\left\|\mathbb{X}^{2}\right\|_{2 \gamma}\right)^{3}
$$

Proof. We prove convergence over the dyadic partition of $[0,1]$, then convergence of a general partition follows then by the arguments developed in [23]. Let $\tau_{\alpha}^{(n)}=T \alpha / 2^{n}$ for $\alpha=0, \ldots, 2^{n}$, and let

$$
S_{n}=\sum_{\alpha=0}^{2^{n}-1}\left(\varphi_{i}\left(\tau_{\alpha}^{(n)}, X_{\tau_{\alpha}^{(n)}}\right) X_{\tau_{\alpha+1}^{(n)} \tau_{\alpha}^{(n)}}^{i}+\nabla_{j} \varphi_{i}\left(\tau_{\alpha}^{(n)}, X_{\tau_{\alpha}^{(n)}}\right) \mathbb{X}_{\tau_{\alpha+1}^{(n)} \tau_{\alpha}^{(n)}}^{i j}\right) .
$$

Note that

$$
S_{n}-S_{n-1}=\sum_{\alpha=0}^{2^{n}-1}\left(A_{\alpha}^{1}+A_{\alpha}^{2}+A_{\alpha}^{3}+A_{\alpha}^{4}\right)
$$

where

$$
\begin{aligned}
& A_{\alpha}^{1}=\left[\varphi_{i}\left(\tau_{2 \alpha+1}^{(n)}, X_{\tau_{2 \alpha+2}^{(n)}}\right)-\varphi_{i}\left(\tau_{2 \alpha+1}^{(n)}, X_{\tau_{2 \alpha+1}^{(n)}}\right)-\nabla_{j} \varphi_{i}\left(\tau_{2 \alpha+1}^{(n)}, X_{\tau_{2 \alpha+1}^{(n)}}\right) X_{\tau_{2 \alpha+2}^{(n)} \tau_{2 \alpha+1}^{(n)}}^{j}\right] X_{\tau_{2 \alpha+1}^{(n)} \tau_{2 \alpha}^{(n)}}^{i}, \\
& A_{\alpha}^{2}=\left[\nabla_{j} \varphi_{i}\left(\tau_{2 \alpha+1}^{(n)}, X_{\tau_{2 \alpha+2}^{(n)}}\right)-\nabla_{j} \varphi_{i}\left(\tau_{2 \alpha+1}^{(n)}, X_{\tau_{2 \alpha+1}^{(n)}}\right)\right] \mathbb{X}_{\tau_{2 \alpha+1}^{(n)} \tau_{2 \alpha}^{(n)}}^{i j}, \\
& A_{\alpha}^{3}=\left[\varphi_{i}\left(\tau_{2 \alpha+2}^{(n)}, X_{\tau_{2 \alpha+2}^{(n)}}\right)-\varphi_{i}\left(\tau_{2 \alpha+1}^{(n)}, X_{\tau_{2 \alpha+2}^{(n)}}\right)\right] X_{\tau_{2 \alpha+1}^{(n)} \tau_{2 \alpha}^{(n)}}^{i}, \\
& A_{\alpha}^{4}=\left(\nabla_{j} \varphi_{i}\left(\tau_{2 \alpha+2}^{(n)}, X_{\tau_{2 \alpha+2}^{(n)}}^{(n)}\right)-\nabla_{j} \varphi_{i}\left(\tau_{2 \alpha+1}^{(n)}, X_{\tau_{2 \alpha+2}^{(n)}}\right)\right) \mathbb{X}_{\tau_{2 \alpha+1}^{(n)} \tau_{2 \alpha}^{(n)}}^{(n)}
\end{aligned}
$$

By using the fact that $\varphi \in C^{2}$, we have the bounds $\left|A_{\alpha}^{1}\right| \leq T^{3 \gamma}\|\varphi\|_{\rho, 2,0, T}\|X\|_{\gamma}^{3} 2^{-3 \gamma n}$ and $\left|A_{\alpha}^{2}\right| \leq T^{3 \gamma}\|\varphi\|_{\rho, 2,0, T}\|X\|_{\gamma}\|\mathbb{X}\|_{2 \gamma} 2^{-3 \gamma n}$ on the first two terms. For the last two we have $\left|A_{\alpha}^{3}\right| \leq T^{\rho+\gamma}\|\varphi\|_{\rho, 2,0, T}\|X\|_{\gamma} 2^{-n(\rho+\gamma)}$ and $\left|A_{\alpha}^{4}\right| \leq T^{\rho+2 \gamma}\|\varphi\|_{\rho, 2,0, T}\|\mathbb{X}\|_{2 \gamma} 2^{-n(\rho+2 \gamma)}$. Thus

$$
\left|S_{n}-S_{n-1}\right| \leq C\left(T^{3 \gamma}+T^{\rho+\gamma}\right)\|\varphi\|_{\rho, 2,0, T}\left(2^{(1-3 \gamma) n}+2^{(1-\gamma-\rho) n}\right)
$$

where $C$ is a function dependent only on $X, \mathbb{X}$. Write $S_{n}$ as the telescopic sum $S_{n}=$ $S_{0}+\sum_{k=1}^{n}\left(S_{k}-S_{k-1}\right)$. Then, since

$$
\sum_{k=1}^{n}\left|S_{k}-S_{k-1}\right| \leq C\left(T^{3 \gamma}+T^{\rho+\gamma}\right)\|\varphi\|_{\rho, 2,0, T} \sum_{k=1}^{n}\left(2^{(1-3 \gamma) n}+2^{(1-\gamma-\rho) n}\right)
$$


converges geometrically whenever $\gamma>1 / 3$ and $\gamma+\rho>1$, we obtain absolute convergence of the sequence $S_{n}$. The bound on the integral thus follows.

As this theorem shows, by using the additional information provided by the path $\mathbb{X}$ we are able to make Riemann sums converge. The following consequence is immediate.

Corollary 2.4 The integral defined in Theorem 2.3 has $\mathbb{X}_{s t}^{i j}=\int_{s}^{t} X_{s u}^{i} d X_{u}^{j}$.

Proof. We have

$$
\int_{s}^{t} X_{u s}^{i} d X_{u}^{j}=\int_{s}^{t} X_{u}^{i} d X_{u}^{j}-X_{s}^{i}\left(X_{u}^{j}-X_{s}^{j}\right)
$$

as it is easily seen by using the definition. That is, the rough integral has the same linearity property as Riemann integral and behaves the same way with respect to the integration of constants. Moreover, the integral in the right hand side corresponds to the function $\varphi_{k}(\xi)=\xi^{i} \delta_{k j}$ and by using (2.4) we have $S_{n}=S_{n-1}$ on the dyadic partition above. Hence $S_{n}=S_{0}$ and

$$
\int_{s}^{t} X_{u}^{i} d X_{u}^{j}=S_{0}=\sum_{k} \varphi_{k}\left(X_{s}\right) X_{s t}^{k}+\sum_{k, m} \nabla_{m} \varphi_{k}\left(X_{s}\right) \mathbb{X}_{s t}^{k m}=X_{s}^{i} X_{s t}^{j}+\mathbb{X}_{s t}^{i j},
$$

which completes the proof.

Remark 2.5 The function $\mathbb{X}$ can be identified as giving the value of a twice iterated integral over $X$,

$$
\mathbb{X}_{s t}^{i j}=\int_{s}^{t}\left(\int_{s}^{u} d X_{v}^{i}\right) d X_{u}^{j}
$$

Thus Theorem 2.3 can be alternatively interpreted as saying that the knowledge of the twice iterated integral (in addition with some Hölder continuity) is sufficient for determining the value of the integral $\int \varphi\left(X_{t}\right) d X_{t}$ for arbitrary $C^{2}$ function.

Provided $\gamma>1 / 2$ and (2.5) holds, whenever the right hand side is defined by using the Young integral, the integral defined in Theorem 2.3 coincides with the Young integral. This is easy to see due to the estimate

$$
\left|\mathbb{X}_{s t}^{i j}\right| \leq C\left(\|X\|_{\infty}+\|X\|_{\gamma}\right)^{2}|t-s|^{2 \gamma}
$$

for the Young integral. Since $2 \gamma>1$, the sums $\sum_{\alpha} \nabla_{j} \varphi_{i}\left(X_{\tau_{\alpha}}\right) \mathbb{X}_{\tau_{\alpha+1} \tau_{\alpha}}^{i j}$ vanish as the mesh goes to zero, so the modified and Riemann sums converge to the same limit. Moreover, the rough integral is continuous in the natural topology associated to the step-2 rough path $(X, \mathbb{X})$, i.e., we have

Corollary 2.6 Let $\left(X^{n}, \mathbb{X}^{n}\right)$ be a sequence of step-2 rough paths such that $\left\|X^{n}-X\right\|_{\gamma}+$ $\left\|\mathbb{X}^{n}-\mathbb{X}\right\|_{2 \gamma} \rightarrow 0$. Then $\int \varphi\left(X^{n}\right) d X^{n} \rightarrow \int \varphi(X) d X$, for all $\varphi \in C^{2}$.

Suppose $X^{n}$ is a sequence of regular (say, piecewise linear) approximations of the Hölder continuous path $X$. Then by putting $\mathbb{X}^{n}=\iint d X^{n} \otimes d X^{n}$, where the integrals are Riemann integrals, a sufficient condition for the convergence of the approximate integrals $\int \varphi\left(X^{n}\right) d X^{n}$ to $\int \varphi(X) d X$ is that the sequence $\mathbb{X}^{n}$ converges to $\mathbb{X}$ in the topology induced by $\|\cdot\|_{2 \gamma}$. 


\subsection{Brownian motion as rough path}

Let now $X$ be a sample path of Brownian motion. By Kolmogorov's Lemma the process $t \mapsto X_{t}$ has a version that is Hölder continuous with exponent $\gamma \in(1 / 3,1 / 2)$ (actually $\gamma$ can be taken arbitrarily close to $1 / 2$ ). In the following we will use such a version without each time mentioning explicitly, i.e., consider a subspace $\mathcal{X}_{\gamma} \subset C\left(\mathbb{R}, \mathbb{R}^{d}\right)$ such that every $X \in \mathcal{X}_{\gamma}$ is Hölder continuous with exponent $\gamma$.

To apply the above results to $X$ we need a choice for $\mathbb{X}$. This candidate is not unique, different choices will lead to different integrals over $X$. For instance, in order to construct a possible $\mathbb{X}$ we can start by setting

$$
\left(\mathbb{X}_{\mathrm{Itô}}\right)_{s t}^{i j}=\int_{s}^{t} \int_{s}^{u} d X_{v}^{i} d X_{u}^{j}
$$

where the double integral is understood in Itô sense. In this way we obtain a family of random variables $\left\{\left(\mathbb{X}_{\mathrm{Ito}}\right)_{s t}^{i j}: i, j=1, \ldots, d ; t, s \in[0, T]\right\}$ satisfying the multiplicative property

$$
\left(\mathbb{X}_{\text {Itô }}\right)_{s t}^{i j}-\left(\mathbb{X}_{\text {Itô }}\right)_{u t}^{i j}-\left(\mathbb{X}_{\text {Itô }}\right)_{s u}^{i j}=X_{u t}^{i} X_{s u}^{j}
$$

almost surely for any fixed $t, s, u \in[0, T]$. The next step is to show that this family has a version for which

$$
\left\|\mathbb{X}_{\text {Itô }}^{i j}\right\|_{2 \gamma}<\infty \quad \text { a.s. }
$$

In order to prove (2.6), we use the following lemma obtained as an extension of a result of Garsia-Rodemich-Rumsey in 23].

Lemma 2.7 For any $\theta>0$ and $p \geq 1$ there exists a constant $C$ such that for any $R \in$ $C\left([0, T]^{2}, \mathcal{B}\right)$, where $(\mathcal{B},|\cdot|)$ is a Banach space, we have

$$
\|R\|_{\theta} \leq C\left(U_{\theta+2 / p, p}(R)+V_{\theta}(R)\right),
$$

with

and

$$
U_{\theta, p}(R)=\left[\int_{[0, T]^{2}}\left(\frac{\left|R_{t s}\right|}{|t-s|^{\theta}}\right)^{p} d t d s\right]^{1 / p}
$$

$$
V_{\theta}(R)=\inf _{\theta_{1} \in(0, \theta)} \sup _{t \neq u \neq s} \frac{\left|R_{s t}-R_{u t}-R_{s u}\right|}{|t-u|^{\theta_{1}}|u-s|^{\theta-\theta_{1}}} .
$$

Corollary 2.8 We have $\left\|\mathbb{X}_{\text {Itô }}\right\|_{2 \gamma}<\infty \mathcal{W}$-almost surely.

Proof. Consider $V_{2 \gamma}\left(\mathbb{X}_{\text {Itô }}\right)$. By using the multiplicative property we have

$$
\begin{aligned}
V_{2 \gamma}\left(\mathbb{X}_{\text {Itô }}\right) & \leq \inf _{\theta_{1} \in(0,2 \gamma)} \sup _{t \neq u \neq s} \frac{\left|X_{t u}\right|\left|X_{u s}\right|}{|t-u|^{\theta_{1}}|u-s|^{2 \gamma-\theta_{1}}} \\
& \leq \inf _{\theta_{1} \in(0,2 \gamma)} \sup _{t \neq u} \frac{\left|X_{t u}\right|}{|t-u|^{\theta_{1}}} \sup _{u \neq s} \frac{\left|X_{u s}\right|}{|u-s|^{2 \gamma-\theta_{1}}} \\
& \leq\left(\sup _{t \neq u} \frac{\left|X_{t u}\right|}{|t-u|^{\gamma}}\right)^{2} \\
& =\|X\|_{\gamma}^{2} .
\end{aligned}
$$


Moreover,

$$
\begin{aligned}
\mathbb{E}\left[U_{2 \gamma, p}\left(\mathbb{X}_{\mathrm{Ito \hat {o }}}\right)^{p}\right] & =\mathbb{E}\left[\int_{[0, T]^{2}}\left(\frac{\left|\left(\mathbb{X}_{\mathrm{It} \hat{\mathrm{o}}}\right)_{t s}\right|}{|t-s|^{2 \gamma}}\right)^{p} d t d s\right] \\
& =\int_{[0, T]^{2}} \frac{\mathbb{E}\left[\left|\left(\mathbb{X}_{\mathrm{Itô}}\right)_{t s}\right|^{p}\right]}{|t-s|^{2 \gamma p}} d t d s,
\end{aligned}
$$

with expectation with respect to Wiener measure. An application of the BurkholderDavis-Gundy inequality [44] allows to estimate the $p$-moment of the double stochastic integral as

$$
\begin{aligned}
\mathbb{E}\left[\mid\left(\mathbb{X}_{\left.\mathrm{Ito \hat { }})\left._{t s}\right|^{p}\right]}\right.\right. & \leq c_{p} \mathbb{E}\left[\int_{s}^{t}\left|X_{u s}\right|^{2} d s\right]^{p / 2} \\
& \leq c_{p}|t-s|^{p / 2-1} \mathbb{E}\left[\int_{s}^{t}\left|X_{u s}\right|^{p} d s\right] \\
& \leq c_{p}^{\prime}|t-s|^{p / 2-1} \int_{s}^{t}|u-s|^{p / 2} d s \\
& \leq c_{p}^{\prime \prime}|t-s|^{p},
\end{aligned}
$$

for all $p>1$ and some $c_{p}, c_{p}^{\prime}, c_{p}^{\prime \prime}>0$. Thus

$$
\mathbb{E}\left[U_{2 \gamma, p}\left(\mathbb{X}_{\mathrm{Itô}}\right)^{p}\right] \leq c_{p}^{\prime \prime} \int_{[0, T]^{2}} \frac{1}{|t-s|^{(2 \gamma-1) p}} d t d s<\infty,
$$

for any $\gamma<1 / 2$, by choosing $p$ large enough $(p>1 /(1-2 \gamma))$.

This last result shows that there exists a version of the stochastic process $\left(X, \mathbb{X}_{\mathrm{Ito}}\right)$ that is a step-2 rough path; from now on we denote by $\left(X, \mathbb{X}_{\text {Itô }}\right)$ this particular version. Then integrals can be defined by applying Theorem 2.3 . We call such an integral rough integral over $\left(X, \mathbb{X}_{\mathrm{Itô}}\right)$.

The relationship between the rough integral and the Itô integral is made clear by

Lemma 2.9 The rough integral over the couple $\left(X, \mathbb{X}_{\text {Itôे }}\right)$ coincides with the Itô integral for any $\varphi \in C^{2}$.

Proof. By Itô theory the sums $\sum_{\alpha} \varphi_{i}\left(X_{\tau_{\alpha}}\right) X_{\tau_{\alpha+1} \tau_{\alpha}}^{i}$ converge in probability to the Itô integral $\int_{0}^{T} \varphi\left(X_{u}\right) d X_{u}$. Hence it suffices to show that the sums $\sum_{\alpha} \nabla_{j} \varphi_{i}\left(X_{\tau_{\alpha}}\right) \mathbb{X}_{\tau_{\alpha+1} \tau_{\alpha}}^{i j}$ converge to zero in $L^{2}$ sense as then it follows that the two integrals almost surely coincide. A simple computation shows that

$$
\mathbb{E}\left[\sum_{\alpha} \nabla_{j} \varphi_{i}\left(X_{\tau_{\alpha}}\right) \mathbb{X}_{\tau_{\alpha+1} \tau_{\alpha}}^{i j}\right]^{2}=\sum_{\alpha} \mathbb{E}\left[\nabla_{j} \varphi_{i}\left(X_{\tau_{\alpha}}\right) \mathbb{X}_{\tau_{\alpha+1} \tau_{\alpha}}^{i j}\right]^{2}
$$

since the cross terms are all zero in the mean by independence of the increments of the Brownian motion. Hence

$$
\mathbb{E}\left[\sum_{\alpha} \nabla_{j} \varphi_{i}\left(X_{\tau_{\alpha}}\right) \mathbb{X}_{\tau_{\alpha+1} \tau_{\alpha}}^{i j}\right]^{2} \leq\|\varphi\|_{1} \sum_{\alpha} \mathbb{E}\left[\mathbb{X}_{\tau_{\alpha+1} \tau_{\alpha}}^{i j}\right]^{2} \leq C\|\varphi\|_{1} \sum_{\alpha}\left|\tau_{\alpha+1}-\tau_{\alpha}\right|^{2}
$$


The last sum vanishes as the mesh of the partition shrinks to zero, thus the claim follows.

In this construction choosing the Itô version for the double integral $\mathbb{X}$ was arbitrary. Alternatively we could have considered other definitions, e.g. Stratonovich integral and let

$$
\left(\mathbb{X}_{\text {Stra }}\right)_{s t}^{i j}=\int_{s}^{t} \int_{s}^{u} \circ d X_{v}^{i} \circ d X_{u}^{j}
$$

where $o d X$ stands for Stratonovich (or symmetric) integration. By the same procedure we find a regular version of $\mathbb{X}_{\text {Stra }}$ such that $\left\|\mathbb{X}_{\text {Stra }}\right\|_{2 \gamma}<\infty$ and can construct the rough integral over the couple $\left(X, \mathbb{X}_{\text {Stra }}\right)$ which we denote again $o d X$. It is not difficult to prove that for $\varphi \in C^{2}$ it coincides with the familiar Stratonovich integral.

The relationship between the rough integrals based on the Itô and Stratonovich constructions follows from the identity

$$
\left(\mathbb{X}_{\text {Stra }}\right)_{s t}^{i j}=\left(\mathbb{X}_{\text {Itô }}\right)_{s t}^{i j}+\frac{1}{2} \delta_{i j}(t-s)
$$

between Itô and Stratonovich stochastic iterated integrals. The correction is given by the increment of the function $t \mapsto \delta_{i j} t / 2$. Thus the two rough integrals are related by the familiar formula

$$
\int_{0}^{T} \varphi\left(X_{u}\right) \circ d X_{u}=\int_{0}^{T} \varphi\left(X_{u}\right) d X_{u}+\frac{1}{2} \int_{0}^{T} \operatorname{div} \varphi\left(X_{u}\right) d u .
$$

This is obtained directly from the definitions with the modified Riemann sums.

Remark 2.10 Due to the multiplicative property the possible choices for $\mathbb{X}$ differ only by the increment of a function, i.e., if $\mathbb{X}_{1}$ and $\mathbb{X}_{2}$ both satisfy the multiplicative property with respect to $X$, then $\left(\mathbb{X}_{1}\right)_{s t}^{i j}-\left(\mathbb{X}_{2}\right)_{s t}^{i j}=f_{t}^{i j}-f_{s}^{i j}$ with a function $f \in C\left([0, T], \mathbb{R}^{d} \times \mathbb{R}^{d}\right)$.

Finally, it is not difficult to see the following regularity result.

Corollary 2.11 The map $\varphi \mapsto \int_{0}^{1} \varphi\left(X_{u}\right) d X_{u}$ is continuous from $C^{2}\left(\mathbb{R}^{d}, \mathbb{R}^{d}\right)$ to $\mathbb{R}$.

\section{Stochastic currents}

\subsection{Lifting Wiener measure to the space of currents}

Let $\mathcal{X}=C\left(\mathbb{R}, \mathbb{R}^{d}\right)$ be path space, i.e., the space of continuous functions from $\mathbb{R}$ to $\mathbb{R}^{d}$. The $\sigma$-field $\mathcal{F}$ is generated by the coordinate process $\mathcal{X} \ni X \mapsto X_{t} \in \mathbb{R}^{d}$. For $I \subset \mathbb{R}$ we denote by $\mathcal{F}_{I}$ the $\sigma$-field over $\mathcal{X}$ generated by the evaluations for points in $I$, and write $\mathcal{F}_{T}$ when $I=[-T, T]$. Also, we put for a shorthand $I^{c}=\mathbb{R} \backslash I$. For $s \leq t$, the set $\left\{\mathcal{F}_{s t}=\sigma\left(X_{u}: s \leq u \leq t\right)\right\}_{s \leq t}$ is the forward filtration starting at $s \in \mathbb{R}$.

Denote as before by $\mathcal{W}$ Wiener measure defined on $\mathcal{X}$ (instead of $C\left([0, \infty), \mathbb{R}^{d}\right)$ as more usual), and write $H_{0}=-(1 / 2) \Delta$. For any finite division $t_{1}<t_{2}<\ldots<t_{n} \in \mathbb{R}$ we have

$$
\mathcal{W}(F)=\left(f_{1}, e^{-\left(t_{2}-t_{1}\right) H_{0}} f_{2} \ldots e^{-\left(t_{n}-t_{n-1}\right) H_{0}} f_{n}\right)_{L^{2}\left(\mathbb{R}^{d}, d x\right)}
$$


where $F=f_{1}\left(X_{t_{1}}\right) \cdots f_{n}\left(X_{t_{n}}\right)$. Here it is understood that $f_{2}, \ldots, f_{n-1}$ act as multiplication operators for which we use the same symbol as for the corresponding functions. The operator $e^{-t H_{0}}$ has the integral kernel

$$
\Pi_{t}(x, y)=\frac{1}{(2 \pi t)^{d / 2}} \exp \left(-\frac{1}{2 t}(x-y)^{2}\right), \quad x, y \in \mathbb{R}^{d} .
$$

We denote by $\mathcal{W}_{I}$ the measure $\mathcal{W}$ restricted to $\mathcal{X}_{I}=C\left(I, \mathbb{R}^{d}\right)$. Similarly, with given $T>0$, $\xi, \eta \in \mathbb{R}^{d}$ write

$$
\mathcal{W}_{I}^{\xi, \eta}(F)=\left(e^{-\left(-T-t_{1}\right) H_{0}} f_{1} e^{-\left(t_{2}-t_{1}\right) H_{0}} f_{2} \ldots e^{-\left(t_{n}-t_{n-1}\right) H_{0}}\left(f_{n} e^{-\left(T-t_{n}\right) H_{0}}(\cdot, \eta)\right)\right)(\xi)
$$

with $I=[-T, T]$ and let $\widehat{\mathcal{W}}_{I}^{\xi, \eta}(F)=\mathcal{W}_{I}^{\xi, \eta}(F) / \Pi_{2 T}(\xi, \eta)$ be the Brownian bridge starting in $\xi$ at $-T$ and ending in $\eta$ at $T$. Under $\widehat{\mathcal{W}}_{[-T, T]}^{x, y}$ the process $X_{t}$ is a Gaussian semimartingale (Brownian bridge) satisfying the SDE

$$
d X_{u}=-\frac{y-X_{u}}{T-u} d u+d B_{u}
$$

where $\left(B_{u}\right)_{u \in[-T, T]}$ is a $\widehat{\mathcal{W}}_{[-T, T]}^{x, y}$-Browian motion.

Next, let $\mathcal{D}$ be a Banach space of functions from $\mathbb{R} \times \mathbb{R}^{d}$ to $\mathbb{R}^{d}$ containing the space of smooth functions $C^{\infty}\left(\mathbb{R} \times \mathbb{R}^{d} ; \mathbb{R}^{d}\right)$. Denote with $\|\cdot\|_{\mathcal{D}}$ the Banach norm. Let $\mathcal{D}^{\prime}$ the topological dual of $\mathcal{D}, \Delta_{I}=\left\{(s, t) \in I^{2} \mid s \leq t\right\}$, and write $\Delta=\Delta_{\mathbb{R}}$.

Definition 3.1 $C \in C\left(\Delta ; \mathcal{D}^{\prime}\right)$ is a stochastic current if it satisfies the following properties:

(1) $C_{t t}(\varphi)=0, \quad C_{s u}(\varphi)+C_{u t}(\varphi)=C_{s t}(\varphi)$, for any $s \leq u \leq t$ and any $\varphi \in \mathcal{D}$;

(2) locality property: $C_{s t}(\varphi)=0$ whenever $\varphi(u, x)=0$ for all $u \in[s, t], x \in \mathbb{R}^{d}$.

We denote $\mathcal{C} \subset C\left(\Delta ; \mathcal{D}^{\prime}\right)$ the space of stochastic currents.

Set $\Xi=\mathcal{X} \times \mathcal{C}$ endowed with the product topology and with the Borel $\sigma$-field (on whose component generated by $\mathcal{C}$ we consider the topology of uniform convergence on bounded intervals). $\Xi$ plays the role of joint path-current configuration space. As a measurable space, it is endowed with a family of $\sigma$-algebras $\left\{\mathcal{A}_{s t}\right\}_{t>s}$ such that $\mathcal{A}_{s t}=\sigma\left(X_{u}, C_{u v}(\varphi)\right.$ : $u, v \in[t, s], \varphi \in \mathcal{D})$. Similarly, we can define the forward filtration $\left\{\mathcal{A}_{t}^{+}\right\}_{t}=\left\{\mathcal{A}_{+\infty, t}\right\}_{t}$ and the backward filtration $\left\{\mathcal{A}_{t}^{-}\right\}_{t}=\left\{\mathcal{A}_{t,-\infty}\right\}_{t}$. The above definitions make sense also in the case that the parameter $t$ is restricted to a bounded interval $I \subset \mathbb{R}$; in this case we denote with $\Xi_{I}$ the corresponding space. Whenever the limits make sense we define $C_{t}^{+}(\varphi)=\lim _{s \rightarrow \infty} C_{t s}(\varphi)$ and $C_{t}^{-}(\varphi)=\lim _{s \rightarrow-\infty} C_{s t}(\varphi)$.

Definition 3.2 $A$ forward current (on $I \subset \mathbb{R}$ ) is a measure $\eta$ on $\Xi$ (on $\Xi_{I}$ ) such that the process $X$ is an $\left(\eta,\left\{\mathcal{F}_{t}^{+}\right\}_{t}\right)$-semimartingale and

$$
C_{s t}^{X}(\varphi)=\int_{s}^{t} \varphi\left(u, X_{u}\right) d X_{u}, \quad \eta-a . s .
$$


for any $(s, t) \in \Delta$ (or $\Delta_{I}$ ) and any adapted $\varphi \in \mathcal{D}$ where on the right hand side we have the standard Itô integral on the semimartingale $X$. When $X$ is Brownian motion, we call $\eta$ (forward) Brownian current.

In order not to multiply terminology, unless confusion may arise we will use the term current also for the elements of $\mathcal{D}^{\prime}$, of $\Xi$, and for the laws on $\Xi$ without making explicit distinction. For unspecified bounded intervals $I$ we use the notation $C_{I}^{X}$ for the associated current with integrator $X$.

Next we want to construct a (non-trivial) measure on $\Xi$ for the Brownian current. Thus we start from Wiener measure $\mathcal{W}$ (similarly we could have worked with the Brownian bridge $\left.\mathcal{W}_{I}^{x, y}\right)$ and prove that there exists a map $F: \mathcal{X} \ni \omega \mapsto F(\omega) \in \mathcal{C}$ such that

$$
F(\cdot)_{s t}(\varphi)=\int_{s}^{t} \varphi\left(u, X_{u}\right) d X_{u}, \quad \mathcal{W} \text {-a.s. }
$$

for any adapted $\varphi \in \mathcal{D}$, with the standard Itô integral at the right hand side. Then a measure $\mathcal{W}^{\sharp}$ on $\Xi$ can be defined as the law of the couple $(X, F)$ under the measure $\mathcal{W}$ and it will be a forward current. The existence of a regular version of map $F$ is an interesting problem in itself which can be addressed by using the techniques developed in [17, 21] for what concerns the regular dependence on $\varphi$. Unfortunately, the topology $\mathcal{D}$ which is implied by such approaches is unsuitable for our applications. Here we prefer to use the theory of rough paths which will provide the necessary regularity for the $F$ map in a more convenient topology.

In Section 2.1 we developed the basic tools of rough-path theory that we need in order to lift Wiener measure to currents. We do this next.

For any $\alpha>1$, let $\mathcal{D}_{\alpha}$ be the completion of the space of smooth test vector fields with respect to the norm

$$
\|\varphi\|_{\mathcal{D}_{\alpha}}=\sup _{k \in \mathbb{Z}}(1+|k|)^{\alpha}\|\varphi\|_{\rho, 2, k, k+1} .
$$

In the following we will fix $\alpha>1$ but otherwise arbitrarily small and write $\mathcal{D}_{\alpha}=\mathcal{D}$.

Lemma 3.3 For any $\gamma \geq 1 / 3, \rho>1-\gamma, t>s$ and $x, y \in \mathbb{R}^{d}$, there exists a family of random variables $F \in \mathcal{C}$ such that (3.4) holds with respect to $\mathcal{W}_{[s, t]}^{x, y}$, and which satisfy the pathwise bound

$$
\left|F_{u v}(\varphi)\right| \leq C_{\gamma, \rho,|t-s|}|u-v|^{\gamma}\left(1+N_{[s, t]}(X)\right)^{3}\|\varphi\|_{\rho, 2, s, t}
$$

for any $\varphi \in \mathcal{D},(u, v) \in \Delta_{[s, t]}$, where $N_{[s, t]}(X)=\left(\|X\|_{\gamma,[s, t]}+\left\|\mathbb{X}^{2}\right\|_{2 \gamma,[s, t]}\right)$ and where $C_{\gamma, \rho,|t-s|}$ depends only on $\gamma, \rho,|t-s|$.

Proof. Define

$$
F_{u v}(\varphi)=\lim _{\delta \tau_{\alpha} \rightarrow 0} \sum_{\alpha}\left(\varphi\left(\tau_{\alpha}, X_{\tau_{\alpha}}\right)+\nabla \varphi\left(\tau_{\alpha}, X_{\tau_{\alpha}}\right) \mathbb{X}_{\tau_{\alpha}, \tau_{\alpha+1}}^{2}\right)
$$

where $\delta \tau_{\alpha}$ is the mesh of the partition and $\mathbb{X}_{s t}^{2} \in C\left(\mathbb{R}^{2} ; \mathbb{R}^{d} \times \mathbb{R}^{d}\right)$ is the twice iterated Itô integral with respect to $X$. By the results on rough path theory in Section 2.1 (compare 
Theorem [2.3), this limit exists whenever $N_{[s, t]}(X)<\infty$ and $\|\varphi\|_{2, \rho, s, t}<\infty$. Otherwise set $F_{u v}(\varphi)=0$. Then $F$ is a well defined random variable obeying (3.6). Moreover, $F_{s u}(\varphi)+F_{u t}(\varphi)=F_{s t}(\varphi)$ and the locality property for $F$ holds by definition.

By straightforward estimates we can also prove that $\mathbb{E}_{\mathcal{W}_{I}^{x, y}}\left[N_{I}(X)^{3}\right]<\infty$ for any $x, y, I$. Using this last result, the equivalence between $F$ and the Itô integral for the adapted vector field $\varphi$ can be proved by the same approach as the one used in the case of Wiener measure.

A direct consequence of Lemma 3.3 is that whenever $N_{[s, t]}(X)<\infty$ the map $\varphi \mapsto$ $F(\omega)_{s t}(\varphi)$ can be considered as an element of $\mathcal{D}^{\prime}$. Moreover if we let

$$
\mathcal{N}_{\alpha, p}(X)=\sum_{k \in \mathbb{Z}}(1+|k|)^{-\alpha} N_{[k, k+1]}(X)^{p},
$$

then whenever $\mathcal{N}_{\alpha, 3}(X)<\infty$, the boundary currents $C_{t}^{+}$and $C_{t}^{-}$are well defined for any $t$ as elements of $\mathcal{D}^{\prime}$.

Lemma 3.4 For every bounded $I \subset \mathbb{R}$ there exists a unique Brownian current $\mathcal{W}_{I}^{\sharp, x, y}$ on $\Xi_{I}$. A similar statement holds for the measures $\mathcal{W}^{\sharp}$ with first marginal $\mathcal{W}$. Moreover, since under $\mathcal{W}$ we have $\mathcal{N}_{\alpha, 3}(X)<\infty$ a.s., the boundary currents are well defined under $\mathcal{W}^{\sharp}$.

Proof. The existence of the lifted measure for Brownian bridge $\mathcal{W}_{I}^{x, y}$ is essentially contained in Lemma 3.3. Its uniqueness is a direct consequence of the property (3.4). The proof in the case of $\mathcal{W}$ is similar and we are left to prove that under $\mathcal{W}^{\sharp}$ the boundary currents are well defined. The integrals in every interval $[i, i+1]$ are well defined, moreover

$$
\left|\int_{i}^{i+1} \varphi\left(u, X_{u}\right) d X_{u}\right| \leq(1+|i|)^{-\alpha}\|\varphi\|_{\mathcal{D}}\left[1+N_{[i, i+1]}(X)\right]^{3}
$$

so that the series $\int_{t}^{+\infty} \varphi\left(u, X_{u}\right) d X_{u}=\sum_{i>t-1} \int_{i \vee t}^{i+1} \varphi\left(u, X_{u}\right) d X_{u}$ is absolutely convergent if $\mathcal{N}_{\alpha, 3}(X)<\infty$. Under $\mathcal{W}$ we have $\mathbb{E}_{\mathcal{W}}\left[\left(1+N_{[i, i+1]}(X)\right)^{3} \mid X_{0}=x\right] \leq C$ uniformly in $i \in \mathbb{Z}$ and $x \in \mathbb{R}^{d}$ thus

$$
\mathbb{E}_{\mathcal{W}}\left[\mathcal{N}_{\alpha, 3}(X) \mid X_{0}=x\right]=\sum_{i \in \mathbb{Z}}(1+|i|)^{-\alpha} \mathbb{E}_{\mathcal{W}}\left[N_{[i, i+1]}(X)^{3} \mid X_{0}=x\right] \leq C \sum_{i \in \mathbb{Z}}(1+|i|)^{-\alpha}<\infty
$$

as soon as $\alpha>1$. This implies that $\mathcal{N}_{\alpha, 3}(X)$ is $\mathcal{W}$-a.s. finite.

Remark 3.5 Note that the lifting of a measure from $\mathcal{X}$ to $\Xi$ is in general not unique. For instance, we could decide to add some other term to the definition of the current,

$$
\tilde{C}_{s t}^{X}(\varphi)=C_{s t}^{X}(\varphi)+\int_{s}^{t} \operatorname{div} \varphi\left(t, X_{t}\right) d t
$$

and obtain a different lifted measure (which is no longer a forward current). It would be interesting to explore whether different lifts may have a different physical meaning in the 
models. For instance, non-relativistic particles with spin can be (partially) described by a current $C^{\sigma}$ defined as

$$
C_{s t}^{X}(\varphi)[\sigma]=C_{s t}^{X}(\varphi)+\int_{s}^{t} \sigma_{t} \cdot \operatorname{curl} \varphi\left(t, X_{t}\right) d t
$$

where $\left(\sigma_{t}\right)_{t}$ is a vector-valued Poisson process describing the spin of the particle [10, 11, 27].

\subsection{Itô current}

Beside defining Brownian currents, it will be useful for our purposes below to define currents for Brownian paths subjected to a potential (or penalty function) $V$. The reason is that we need a sufficiently strong confining mechanism of paths in order to investigate the effect of a pair interaction on them (given by double stochastic integrals). The translation invariant $(V \equiv 0)$ regime is presently little understood.

While we properly introduce potentials only in the next section, we require here that the integral kernel

$$
\hat{\pi}_{t-s}(x, y)=\int e^{-\int_{s}^{t} V\left(X_{u}\right) d u} d \mathcal{W}_{[s, t]}^{x, y}(X), \quad \forall s<t \in \mathbb{R}, \forall x, y \in \mathbb{R}^{d}
$$

exists. With assumptions on $V$ listed in Section 4.1 below this can be ensured, and then furthermore the map $(t, x, y) \mapsto \hat{\pi}_{t}(x, y)$ is jointly continuous and bounded on $(0, \infty) \times$ $\mathbb{R}^{d} \times \mathbb{R}^{d}$ giving rise to a semigroup $S_{t}$ via the formula

$$
\left(S_{t} f\right)(x)=\int \hat{\pi}_{t}(x, y) f(y)
$$

Moreover, for every $t>0$ the semigroup $S_{t}$ is a bounded operator from $L^{p}$ to $L^{q}$ for every $1 \leq p \leq q \leq \infty$, and by the Feynman-Kac formula and the Hille-Yoshida and Stone Theorems it can be written as $S_{t}=e^{-t H}$, with $H$ coinciding with the Schrödinger operator $H_{0}+V$ on $C_{0}^{\infty}\left(\mathbb{R}^{d}\right)$. In addition, $e^{-t H} f$ is a continuous function for every $f \in L^{p}, p \in[1, \infty]$, $\forall t>0$.

Let $\Psi$ be a ground state of the Schrödinger operator $H$, i.e., a normalized eigenfunction in $L^{2}\left(\mathbb{R}^{d}, d x\right)$ lying at inf $\operatorname{Spec} H$. Under the conditions given in Section 4.1. this ground state is unique and has a strictly positive version. Using this we define the probability measure $\nu$ on $(\mathcal{X}, \mathcal{F}, \mathcal{W})$ by

$$
\nu(A)=Z_{T}^{-1} \int d x \Psi(x) \int d y \Psi(y) \int 1_{A}(X) e^{-\int_{-T}^{T}\left(X_{t}\right) d t} d \mathcal{W}_{T}^{x, y}(X)
$$

with normalizing constant $Z_{T}, A \in \mathcal{F}_{T}$. This measure can be extended to a measure $\nu$ on the full $\mathcal{F}$ by making use of the facts $e^{-t H} \Psi=\Psi$ and $\|\Psi\|_{2}=1$. The FeynmanKac formula and the Markov property of Brownian motion imply that $\left\{\nu_{T}\right\}$ given on $\mathcal{F}_{T}, T>0$, define a consistent family of probability measures. In particular, $\nu$ satisfies the DLR equations and thus is a Gibbs measure relative to Brownian motion for potential $V$; for further details see [4]. 
Moreover, $\nu$ is the law of a reversible diffusion process with stationary distribution $d \omega=\Psi^{2} d x$ and stochastic generator $H_{\omega}$ acting in $L^{2}\left(\mathbb{R}^{d}, d \omega\right)$ as

$$
H_{\omega} f=\frac{1}{\Psi} H(\Psi f)=H_{0} f-(\nabla \log \Psi, \nabla f)_{\mathbb{R}^{d}} .
$$

This process is called Itô diffusion (or $P(\phi)_{1}$-process in quantum field theory). Its transition probabilities are given by

$$
\nu\left(f\left(X_{t+s}\right) \mid X_{s}=x\right)=\int \pi_{t}(x, y) f(y) d \omega(y),
$$

where

$$
\pi_{t}(x, y)=\frac{\hat{\pi}_{t}(x, y)}{\Psi(x) \Psi(y)}
$$

is the transition density of $\nu$ with respect to its stationary distribution. The Itô process is Markovian, reversible, and has a version with continuous paths. Moreover, it is a Brownian semi-martingale with respect to either the forward or the backward filtration, in particular it is the stationary solution of the forward stochastic differential equation

$$
d X_{t}=\nabla \log \Psi\left(X_{t}\right) d t+d B_{t},
$$

where $B_{t}$ is Brownian motion with respect to the forward filtration.

For the Itô bridge, i.e., the regular conditional probability $\nu_{T}^{x, y}$ of $\nu$ given $X_{-T}=x$, $X_{T}=y$, we will use the following representation. Take (3.8) describing the density of the measure $\nu_{T}$ with respect to Brownian motion. Then

$$
\nu_{T}^{x, y}(A)=\frac{\Pi_{2 T}(x, y)}{Z_{T} \Psi(x) \Psi(y) \pi_{2 T}(x, y)} \mathbb{E}_{\widehat{\mathcal{W}}_{[-T, T]}^{x, y}}\left[1_{A}(X) e^{-V_{[-T, T]}(X)}\right],
$$

with $\Pi_{t}$ the Brownian transition kernel (3.2). This formula can be checked by noting that

$$
\begin{aligned}
\hat{\mathbb{E}}_{\nu_{T}} & {\left[f(x) g(y) \mathbb{E}_{\nu_{T}^{x, y}}[Q]\right] } \\
& =Z_{T}^{-1} \hat{\mathbb{E}}_{\mathcal{W}_{[-T, T]}}\left[f(x) g(y) \Psi(x) \Psi(y) \mathbb{E}_{\widehat{\mathcal{W}}_{[-T, T]}^{x, y}}\left[Q(X) e^{-\int_{-T}^{T} V\left(X_{t}\right) d t}\right]\right] \\
& =Z_{T}^{-1} \mathbb{E}_{\mathcal{W}_{[-T, T]}}\left[\Psi\left(X_{-T}\right) \Psi\left(X_{T}\right) f\left(X_{T}\right) g\left(X_{-T}\right) Q(X) e^{-\int_{-T}^{T} V\left(X_{t}\right) d t}\right] \\
& =\mathbb{E}_{\nu_{T}}\left[f\left(X_{T}\right) g\left(X_{-T}\right) Q(X)\right]
\end{aligned}
$$

where $\hat{\mathbb{E}}$ denotes the expectation in a new probability space whose coordinate process is denoted $\hat{X}$, and with $\hat{X}_{-T}=x, \hat{X}_{T}=y$.

The assumption that $V$ is Kato-class (see Section 4.1) implies that the Itô bridge measure $\nu_{I}^{x, y}$ is absolutely continuous with respect to the Brownian bridge measure $\mathcal{W}_{I}^{x, y}$. This allows us to use the lifting result proved in Lemma 3.4 to show that also $\nu_{I}^{x, y}$ allows a lift to the space of currents. 
Lemma 3.6 For every bounded $I \subset \mathbb{R}$ there exists a forward current $\nu_{I}^{\sharp, x, y}$ on $\Xi_{I}$ such that is first marginal is $\nu_{I}^{x, y}$. A similar statement holds for the stationary measures $\nu^{\sharp}$ with first marginal $\nu$. Moverover, since under $\nu$ we have $\mathcal{N}_{\alpha, 3}(X)<\infty$ a.s., the boundary currents are well defined under $\nu^{\sharp}$.

Proof. We have

$$
\mathbb{E}_{\nu_{I}^{x, y}}\left[N_{I}(X)^{3}\right] \leq C\left(\mathbb{E}_{\widehat{\mathcal{W}}_{I}^{x, y}}\left[N_{I}(X)^{6}\right]\right)^{1 / 2}\left(\mathbb{E}_{\nu_{I}^{x, y}}\left[e^{-2 \int_{I} V\left(X_{t}\right) d t}\right]\right)^{1 / 2} \leq C .
$$

Hence the map $F$ defined in Lemma 3.3 is well defined and coincides almost surely with the Itô integral. This allows to construct the lifted measures $\nu_{I}^{\sharp, x, y}$ and $\nu^{\sharp}$. Moreover, by using stationarity of $\nu$ we have that $\mathbb{E}_{\nu}\left[N_{[i, i+1]}(X)^{3}\right] \leq C$ uniformly in $i \in \mathbb{Z}$, thus the boundary currents are well defined under $\nu^{\sharp}$.

\section{Gibbs measures on Brownian currents}

\subsection{Conditions on the potentials}

We use the same terminology of the usual DLR theory and introduce "potentials" and "energy functionals" below.

An external potential is a Lebesgue measurable function $V: \mathbb{R}^{d} \rightarrow \mathbb{R}$ that we will choose from the Kato class, i.e., an element of the space $\mathcal{K}\left(\mathbb{R}^{d}\right)$ defined by the condition

$$
\lim _{r \rightarrow 0} \sup _{x \in \mathbb{R}^{d}} \int_{B_{r}(x)}|g(y-x) V(y)| d y=0,
$$

with $B_{r}(x)$ the ball of radius $r$ centered at $x$, and

$$
g(x)= \begin{cases}|x| & \text { if } d=1 \\ -\ln |x| & \text { if } d=2 \\ |x|^{2-d} & \text { if } d \geq 3\end{cases}
$$

This space is large enough to contain many choices of interest, while allowing the FeynmanKac formula for the Schrödinger semigroup $e^{-t H}, t \geq 0$, to hold. This is generated by the Schrödinger operator $H=H_{0}+V$ defined on $L^{2}\left(\mathbb{R}^{d}, d x\right)$ as a form sum ( $V$ regarded as a multiplication operator). For Kato-class potentials $H$ is essentially self-adjoint on the form core $C_{0}^{\infty}\left(\mathbb{R}^{d}\right)$. In addition, we will require of $V$ to be such that

(1) $H$ has a unique strictly positive eigenfunction (ground state) $\Psi$ at $E=\inf \operatorname{Spec} H$, with the property that $\Psi \in L^{1} \cup L^{\infty}$;

(2) $e^{-t H}$ is intrinsically ultracontractive.

Recall the meaning of the latter property. Write $d \omega=\Psi^{2} d x$ on $\mathbb{R}^{d}$ as before, and define the isometry (ground state transform) $j: L^{2}\left(\mathbb{R}^{d}, d \omega\right) \rightarrow L^{2}\left(\mathbb{R}^{d}, d x\right), f \mapsto \Psi f$. Then $D\left(H_{\omega}\right)=j^{-1} D(H)$ and $H_{\omega} f=\left(j^{-1} H j\right) f=(1 / \Psi) H(\Psi f)=-(1 / 2) \Delta f-(\nabla \ln \Psi, \nabla f)_{\mathbb{R}^{d}}$, for every $f \in D\left(H_{\omega}\right)$. The associated semigroup $e^{-t H_{\omega}}$ exists for all $f \in L^{2}\left(\mathbb{R}^{d}, d \omega\right)$ and 
$t \geq 0$. $e^{-t H}$ is intrinsically ultracontractive when $e^{-t H_{\omega}}$ is ultracontractive, i.e., it maps $L^{2}\left(\mathbb{R}^{d}, d \omega\right)$ into $L^{\infty}\left(\mathbb{R}^{d}, d \omega\right)$ continuously. Equivalently, this means that $\left\|e^{-t H_{\omega}}\right\|_{2, \infty}<\infty$, $\forall t>0$, and it is a monotonically decreasing function in $t$. Moreover, the integral kernel (3.10) of $e^{-t H_{\omega}}$ satisfies $0 \leq \pi_{t}(x, y) \leq\left\|e^{-(t / 2) H_{\omega}}\right\|_{2, \infty}^{2}$ almost surely.

These conditions are in particular satisfied for $V$ bounded from below, continuous, and sufficiently confining, i.e., for which there exist constants $C_{1}, C_{2}>0, C_{3}, C_{4} \in \mathbb{R}$, and $a, b$ with $2<a<b<2 a-2$ such that the positive part of the potential, $V^{+}=\sup \{0, V\}$ satisfies

$$
C_{1}|x|^{a}+C_{3} \leq V^{+}(x) \leq C_{2}|x|^{b}+C_{4}
$$

A pair interaction potential is a Lebesgue measurable function $W: \mathbb{R}^{d} \times \mathbb{R} \rightarrow \mathbb{R}$, even in both of its variables, which we require to

(1) have positive Fourier transform;

(2) satisfy the regularity condition that there exists $M_{I, \beta} \in \mathbb{R}$ such that

$$
\sup _{x \in \mathbb{R}^{d}, t \in I}\|W(x, t)\|_{\mathcal{D}_{\beta}} \leq M_{I, \beta},
$$

for $\beta>\max \{\alpha, 3\}$ and every bounded $I \subset \mathbb{R}$.

The requirement $\beta>\alpha$ is needed for having a well defined interaction energy (actually in our applications for any $\beta>1$ there is a suitable $\alpha$ so that this holds), while $\beta>3$ is a decay condition sufficient for ensuring the convergence of the cluster expansion in Chapter 5 below.

An example satisfying these conditions is $W^{\rho}$ seen in Section 1.

Finally we write down the energies appearing in the definition of the densities of Gibbs measures we are going to study. With given pair potential $W$, for all $a, b \in \mathcal{D}^{\prime}$ consider the function $\psi_{k, \varpi}(x, t)=e^{i k \cdot x+i \varpi t} \in \mathcal{D}$ and define the (possibly unbounded) quadratic form

$$
\langle a, b\rangle_{W}:=\int \widehat{W}(k, \varpi) a\left(\psi_{k, \varpi}\right) b\left(\overline{\psi_{k, \varpi}}\right) d k d \varpi
$$

By using the quadratic form, for all bounded $I \subset \mathbb{R}$ and every $\mathbb{X}=\left(X, C^{X}\right), \mathbb{Y}=\left(Y, C^{Y}\right) \in$ $\Xi$ define the internal energy functional

$$
H_{I}(\mathbb{X})=V_{I}(X)+\frac{\lambda}{2}\left\langle C_{I}^{X}, C_{I}^{X}\right\rangle_{W},
$$

and interaction functional

$$
H_{I}(\mathbb{X} \mid \mathbb{Y})=V_{I}(X)+\frac{\lambda}{2}\left\langle C_{I}^{X}, C_{I}^{X}\right\rangle_{W}+\lambda\left\langle C_{I}^{X}, C_{I^{c}}^{Y}\right\rangle_{W},
$$

with parameter $\lambda \in \mathbb{R}$, where we wrote $V_{I}(X)=\int_{I} V\left(X_{t}\right) d t$. 


\subsection{Gibbs specifications}

On $\Xi$ with its associated $\sigma$-Borel field $\mathcal{A}$ we take now $\mathcal{W}^{\sharp}$ as reference measure and define a Gibbs specification.

Definition 4.1 (Gibbs specification) Take the regular version $\mathcal{W}_{I}^{\sharp}(d \mathbb{X} \mid \mathbb{Y})$ of the measure $\mathcal{W}^{\sharp}$ conditional on $\mathbb{Y}$ in $\mathcal{A}_{I^{c}}$, and $H_{I}(\mathbb{X})$ given by 4.5$)$. We call the family of probability kernels $\left\{\mu_{I}^{\sharp}\right\}_{I}$ on $\Xi$ indexed by the bounded intervals $I \subset \mathbb{R}$,

$$
\mu_{I}^{\sharp}(d \mathbb{X})=\frac{e^{-H_{I}(\mathbb{X})}}{Z_{I}} \mathcal{W}_{I}^{\sharp}(d \mathbb{X})
$$

$a$ Gibbs specification on Brownian currents with free boundary condition. Take $H_{I}(\mathbb{X} \mid \mathbb{Y})$ given by (4.6). We call the family $\left\{\rho_{I}^{\sharp}\right\}_{I}$ on $\Xi$,

$$
\rho_{I}^{\sharp}(d \mathbb{X} \mid \mathbb{Y})=\frac{e^{-H_{I}(\mathbb{X} \mid \mathbb{Y})}}{Z_{I}(\mathbb{Y})} \mathcal{W}_{I}^{\sharp}(d \mathbb{X} \mid \mathbb{Y})
$$

$a$ Gibbs specification on Brownian currents with boundary condition $\mathbb{Y}$.

Definition 4.2 (Gibbs measure) A probability measure $\mu$ on $\left(\Xi, \mathcal{A}, \mathcal{W}^{\sharp}\right)$ is a Gibbs measure for the potentials $V$ and $W$ if it is consistent with the specification $\left\{\rho_{I}^{\sharp}\right\}_{I}$, i.e., there exists a version of its conditional probabilities with respect to the family $\left\{\mathcal{A}_{I^{c}}\right\}_{I}$ which agrees with $\left\{\rho_{I}^{\sharp}\right\}_{I}$ for all bounded $I \subset \mathbb{R}$.

In the following chapter our main concern will be to prove the existence of such Gibbs measures.

On Gibbs specifications here is a first result.

Lemma 4.3 The family $\left\{\rho_{I}^{\sharp}\right\}_{I}$ is consistent, i.e., for every pair of bounded intervals $I \subset J \subset \mathbb{R}$ we have $\iint F(\mathbb{X}) \rho_{I}^{\sharp}(d \mathbb{X} \mid \mathbb{Y}) \rho_{J}^{\sharp}(d \mathbb{Y} \mid \mathbb{Z})=\int F(\mathbb{X}) \rho_{J}^{\sharp}(d \mathbb{X} \mid \mathbb{Z})$, for any bounded measurable $F: \Xi \rightarrow \mathbb{R}$.

Proof. The family $\left\{\mathcal{W}_{I}^{\sharp}\right\}_{I}$ is consistent by its definition. Hence

$$
\begin{aligned}
\iint F(\mathbb{X}) \rho_{I}^{\sharp}(d \mathbb{X} \mid \mathbb{Y}) \rho_{J}^{\sharp}(d \mathbb{Y} \mid \mathbb{Z}) \\
=\iint F(\mathbb{X}) \frac{e^{-V_{I}(X)-(\lambda / 2)\left\langle C_{I}^{X}, C_{I}^{X}\right\rangle_{W}-\lambda\left\langle C_{I}^{X}, C_{I^{c}}^{Y}\right\rangle_{W}}}{Z_{I}(\mathbb{Y})} \\
\quad \times \frac{e^{-V_{J}(Y)-(\lambda / 2)\left\langle C_{J}^{Y}, C_{J}^{Y}\right\rangle_{W}-\lambda\left\langle C_{J}^{Y}, C_{J}^{Z}\right\rangle_{W}}}{Z_{J}(\mathbb{Z})} \mathcal{W}_{I}^{\sharp}(d \mathbb{X} \mid \mathbb{Y}) \mathcal{W}_{J}^{\sharp}(d \mathbb{Y} \mid \mathbb{Z}) \\
=\iint F(\mathbb{X}) \frac{e^{-V_{I}(X)-(\lambda / 2)\left\langle C_{I}^{X}, C_{I}^{X}\right\rangle_{W}-\lambda\left\langle C_{I}^{X}, C_{I}^{Y}\right\rangle_{W}}}{Z_{I}(\mathbb{Y})} \\
\quad \times e^{-V_{I}(Y)-(\lambda / 2)\left\langle C_{I}^{Y}, C_{I}^{Y}\right\rangle_{W}-\lambda\left\langle C_{I}^{Y}, C_{J}^{Z}\right\rangle_{W}-\lambda\left\langle C_{I}^{Y}, C_{K}^{Y}\right\rangle_{W}} \\
\quad \times \frac{e^{-V_{K}(Y)-(\lambda / 2)\left\langle C_{K}^{Y}, C_{K}^{Y}\right\rangle_{W}-\lambda\left\langle C_{K}^{Y}, C_{J c}^{Z}\right\rangle_{W}}}{Z_{J}(\mathbb{Z})} \mathcal{W}_{I}^{\sharp}(d \mathbb{X} \mid \mathbb{Y}) \mathcal{W}_{J}^{\sharp}(d \mathbb{Y} \mid \mathbb{Z})
\end{aligned}
$$


where we split off $C_{J}^{Y}=C_{I}^{Y}+C_{K}^{Y}$ with $K=J \backslash I$. This gives for the right hand side

$$
\begin{aligned}
\iint & F(\mathbb{X}) \frac{e^{-V_{I}(X)-(\lambda / 2)\left\langle C_{I}^{X}, C_{I}^{X}\right\rangle_{W}-\lambda\left\langle C_{I}^{X}, C_{I}^{Y}\right\rangle_{W}}}{Z_{I}\left(\mathbb{Y}_{2}\right)} \\
& \times\left(\int e^{-V_{I}\left(Y_{1}\right)-(\lambda / 2)\left\langle C_{I}^{Y_{1}}, C_{I}^{Y_{1}}\right\rangle_{W}-\lambda\left\langle C_{I}^{Y_{1}}, C_{J c}^{Z}\right\rangle_{W}-\lambda\left\langle C_{I}^{Y_{1}}, C_{K}^{Y_{2}}\right\rangle_{W}} \mathcal{W}_{I}^{\sharp}\left(d \mathbb{Y}_{1} \mid \mathbb{Y}_{2}\right)\right) \\
& \times \frac{e^{-V_{K}\left(Y_{2}\right)-(\lambda / 2)\left\langle C_{K}^{Y_{2}}, C_{K}^{Y_{2}}\right\rangle_{W}-\lambda\left\langle C_{K}^{Y_{2}}, C_{J^{c}}^{Z}\right\rangle_{W}}}{Z_{J}(\mathbb{Z})} \mathcal{W}_{I}^{\sharp}\left(d \mathbb{X} \mid \mathbb{Y}_{2}\right) \mathcal{W}_{K}^{\sharp}\left(d \mathbb{Y}_{2} \mid \mathbb{Z}\right),
\end{aligned}
$$

where we used the fact that $\int \mathcal{W}_{I}^{\sharp}\left(d \mathbb{Y}_{1} \mid \mathbb{Y}_{2}\right) \mathcal{W}_{K}^{\sharp}\left(d \mathbb{Y}_{2} \mid \mathbb{Z}\right)=\mathcal{W}_{J}^{\sharp}\left(d \mathbb{Y}_{1} \mid \mathbb{Z}\right)$. Note that the expression between the brackets equals $Z_{I}\left(\mathbb{Y}_{2}\right)$, thus we further obtain

$$
\begin{aligned}
& \iint F(\mathbb{X}) e^{-V_{I}(X)-(\lambda / 2)\left\langle C_{I}^{X}, C_{I}^{X}\right\rangle_{W}-\lambda\left\langle C_{I}^{X}, C_{I^{c}}^{Y}\right\rangle_{W}} \\
& \quad \times \frac{e^{-V_{K}\left(Y_{2}\right)-(\lambda / 2)\left\langle C_{K}^{Y_{2}}, C_{K}^{Y_{2}}\right\rangle_{W}-\lambda\left\langle C_{K}^{Y_{2}}, C_{J}^{Z}\right\rangle_{W}}}{Z_{J}(\mathbb{Z})} \mathcal{W}_{I}^{\sharp}\left(d \mathbb{X} \mid \mathbb{Y}_{2}\right) \mathcal{W}_{K}^{\sharp}\left(d \mathbb{Y}_{2} \mid \mathbb{Z}\right) \\
& =\int F(\mathbb{Y}) \frac{e^{-V_{J}(Y)-(\lambda / 2)\left\langle C_{J}^{Y}, C_{J}^{Y}\right\rangle_{W}-\lambda\left\langle C_{J}^{Y}, C_{J c}^{Z}\right\rangle_{W}}}{Z_{J}(\mathbb{Z})} \mathcal{W}_{J}^{\sharp}(d \mathbb{Y} \mid \mathbb{Z})=\int F(\mathbb{Y}) \rho_{J}(d \mathbb{Y} \mid \mathbb{Z}) .
\end{aligned}
$$

The forward current $\mathcal{W}^{\sharp}$ has the key property that $C_{s t}^{X}(\varphi)=\int_{s}^{t} \varphi\left(u, X_{u}\right) d X_{u}, \mathcal{W}^{\sharp}-$ a.s. for all $(s, t) \in \Delta$ and all adapted $\varphi \in \mathcal{D}$. This will enable us to show that the finite volume measures $\mu_{I}$ coincide with the marginals of the measures $\mu_{I}^{\sharp}$ on the first component of the product $\Xi$. The specification $\left\{\rho_{I}^{\sharp}\right\}$ can then be considered as a suitable rigorous replacement for the DLR description of the infinite-volume limit. A Gibbs measure on $\mathcal{X}$ will then be a measure for which there exists a unique lift to the space $\Xi$ of currents satisfying the relation (3.4) ensuring the identification of the current with the stochastic integral and which satisfy the DLR conditions with respect to the specification $\left\{\rho_{I}^{\sharp}\right\}$.

To show that the specification is well defined we rewrite the various terms using the fact that, under the measure $\mathcal{W}_{I}^{\sharp}(d \mathbb{X} \mid \mathbb{Y})$ we have pathwise equality between the current $C^{X}$ and the stochastic integral with respect to $X$ for adapted integrands belonging to $\mathcal{D}$. Then

$$
\begin{aligned}
\left\langle C_{I}^{X}, C_{I}^{X}\right\rangle_{W} & =\int \widehat{W}(k, \varpi) C_{I}^{X}\left(\psi_{k, \varpi}\right) C_{I}^{X}\left(\overline{\psi_{k, \varpi}}\right) d k d \varpi \\
& =\int \widehat{W}(k, \varpi)\left|C_{I}^{X}\left(\psi_{k, \varpi}\right)\right|^{2} d k d \varpi \\
& =\int \widehat{W}(k, \varpi)\left|\int_{I} \psi_{k, \varpi}\left(t, X_{t}\right) d X_{t}\right|^{2} d k d \varpi=2 W_{I}(X)
\end{aligned}
$$


and

$$
\begin{aligned}
\left\langle C_{I}^{X}, C_{I^{c}}^{Y}\right\rangle_{W} & =\int \widehat{W}(k, \varpi) C_{I}^{X}\left(\psi_{k, \varpi}\right) C_{I^{c}}^{Y}\left(\overline{\psi_{k, \varpi}}\right) d k d \varpi \\
& =C_{I}^{X}\left(\int \widehat{W}(k, \varpi) \psi_{k, \varpi} C_{I^{c}}^{Y}\left(\overline{\psi_{k, \varpi}}\right) d k d \varpi\right) \\
& =C_{I}^{X}\left(w^{C_{I^{c}}^{Y}}\right)=\int_{-T}^{T} C_{I^{c}}^{Y}\left(W\left(\cdot-X_{s}, \cdot-s\right)\right) d X_{s}
\end{aligned}
$$

with

$$
w^{C}(x, t)=\int \widehat{W}(k, \varpi) \psi_{k, \varpi}(t, x) C\left(\overline{\psi_{k, \varpi}}\right) d k d \varpi=C(W(x-\cdot, t-\cdot)) .
$$

By using these equalities it is seen that the specification (4.8) takes the form

$$
\rho_{I}^{\sharp}(d \mathbb{X} \mid \mathbb{Y})=\frac{e^{-V_{I}(X)-\lambda W_{I}(X)-\lambda \int_{I} w^{C_{I^{c}}^{Y}}\left(u, X_{u}\right) d X_{u}}}{Z_{I}(\mathbb{Y})} \mathcal{W}_{I}^{\sharp}(d \mathbb{X} \mid \mathbb{Y})
$$

and it is well defined as soon as the exponential weight is integrable and the integral is different from zero. The conditions on $V$ and $W$ make sure this is true. Indeed, for Kato-class potentials exponential integrability is a consequence of Khasminskii's Lemma [47. Moreover, since the Fourier transform of $W$ is positive by assumption and $\lambda>0$, the internal energy term is negative and thus exponentially integrable without any further restriction. For the interaction with the boundary current we have

$$
\left|w^{C_{I^{c}}^{Y}}(x, t)\right|=\left|C_{I^{c}}^{Y}(W(x, t))\right| \leq M_{I}\left\|C_{I^{c}}^{Y}\right\|_{\mathcal{D}^{\prime}}
$$

with $M_{I}=\sup _{x \in \mathbb{R}^{d}, t \in I}\|W(x, t)\|_{\mathcal{D}}$, which by condition (2) on $W$ is finite. Hence the stochastic integral in the exponent has a bounded and adapted integrand and thus by standard techniques it follows that it is exponentially integrable for any value of $\lambda$.

By making use of the Itô current defined in Section 3.2 , the specification $\left\{\rho_{I}^{\sharp}\right\}_{I}$ can be finally written as

$$
\rho_{I}^{\sharp}(d \mathbb{X} \mid \mathbb{Y})=\frac{e^{-\lambda W_{I}(X)-\lambda \int_{-T}^{T} w^{C_{I}^{Y}}\left(u, X_{u}\right) d X_{u}}}{\mathcal{Z}_{I}(\mathbb{Y})} \nu_{I}^{\sharp}(d \mathbb{X} \mid \mathbb{Y}) .
$$

Note that this is a forward current on $\Xi$ but by the above results it can be obtained as the unique lift of its marginal on $\mathcal{X}$ satisfying the identification (3.4) between currents and stochastic integrals.

Remark 4.4 The specification (4.9) seems to depend only on the path and the currents appearing in the definition of the vector-field $w^{C}$ that describes the interaction with the boundary paths. The point of introducing measures and specifications on currents resides in the fact that we are not able to describe (4.9) in terms of paths alone. The framework of stochastic currents is not the only possibility to solve this difficulty. A different way to proceed is considering directly rough paths and defining the measures and specifications 
on the space of (step-2) rough paths, i.e., formally of couples $\left(X, \mathbb{X}^{2}\right)$, where $\mathbb{X}^{2}$ is the twice iterated integral associated with the paths $X$. This would solve the problem of stochastic integrals, which can then be defined as rough integrals, and with suitable growth conditions on the rough paths we would be also allowed to define the interaction terms with boundary paths (over unbounded time intervals) and specifications similarly to that on the currents. Our approach is motivated essentially by the consideration that currents are more basic objects than rough paths. We prefer to see rough path theory as a tool for obtaining stochastic currents in useful topologies. Indeed, in principle the construction of good versions of stochastic integrals can be carried out without recourse to rough paths [17, 21].

\section{Existence of Gibbs measures for Brownian currents}

\subsection{Cluster representation}

In the following we will construct a Gibbs measure that is consistent with the specification $\left\{\rho_{I}^{\sharp}\right\}_{I}$. This will be achieved by breaking up paths according to a sequence of bounded subintervals of the real line, and constructing Gibbs measures for bounded intervals. Taking limits over these Gibbs measures will result in a Gibbs measure on $\mathcal{X}$ whose lifted measure to $\Xi$ is consistent with the given specification. As mentioned before, a reasonably confining $V$ is needed to make sure that the paths are not allowed to escape to infinity with large probability.

The following notion of convergence will be used below to discuss Gibbs measures. Let generally $E$ be a metric space, and $C(\mathbb{R}, E)$ the space of continuous paths $\left\{X_{t} ; t \in \mathbb{R}\right\}$ with values in $E$. For any bounded interval $I \subset \mathbb{R}$ let $\mathcal{E}_{I} \subset \mathcal{E}$ be a sub- $\sigma$-field of the Borel $\sigma$-field $\mathcal{E}$ of $E$ generated by the evaluations $\left\{X_{t}: t \in I\right\}$. A sequence of probability measures $\left(m_{n}\right)_{\{n \in \mathbb{N}\}}$ on $C(\mathbb{R}, E)$ is said to converge locally weakly to the probability measure $m$ if for any such $I$ the restrictions $\left.m_{n}\right|_{\mathcal{E}_{I}}$ converge weakly to the measure $\left.m\right|_{\mathcal{E}_{I}}$.

The main result of this paper is the following

Theorem 5.1 Suppose $V$ and $W$ satisfy the assumptions stated in Section 4.1. Take any unbounded increasing sequence $\left(T_{n}\right)_{n \geq 0}$ of positive real numbers, and suppose $0<|\lambda| \leq \lambda^{*}$ with $\lambda^{*}$ small enough. Then the local weak limit $\lim _{n \rightarrow \infty} \mu_{T_{n}}=\mu$ exists on $\mathcal{X}$ and does not depend on the choice of sequence $T_{n}$. Its unique lift $\mu^{\sharp}$ on $\Xi$ is a Gibbs probability measure consistent with the specification $\left\{\rho_{I}^{\sharp}\right\}_{I}$.

Proof. We develop a cluster expansion, i.e., choose the coupling parameter $\lambda$ sufficiently small for being able to control the measure for the interaction switched on $(\lambda \neq 0)$ in terms of a convergent perturbation series around the free case $(\lambda=0)$. The theorem follows then through Propositions 5.2, 5.3, 5.5 and 5.11 below.

Take a division of $[-T, T]$ into disjoint intervals $\tau_{k}=\left(t_{k}, t_{k+1}\right), k=0, \ldots, N-1$, with $t_{0}=-T$ and $t_{N}=T$, each of length $b$, i.e. fix $b=2 T / N$; for convenience we choose $N$ to be an even number so that the origin is endpoint to some intervals. We break up a path $X$ into pieces $X_{\tau_{k}}$ by restricting it to $\tau_{k}$. The total energy contribution of the pair 
interaction then becomes

$$
W_{T}(X)=\frac{1}{2} \sum_{i, j=0}^{N-1}\left\langle C_{\tau_{i}}^{X}, C_{\tau_{j}}^{X}\right\rangle_{W}=\sum_{0 \leq i<j \leq N-1} W_{\tau_{i}, \tau_{j}}
$$

where with the notation $\mathcal{J}_{i j}=\left\langle C_{\tau_{i}}^{X}, C_{\tau_{j}}^{X}\right\rangle_{W}$ we have

$$
W_{\tau_{i}, \tau_{j}}= \begin{cases}\mathcal{J}_{i j}+\mathcal{J}_{j i} & \text { if }|i-j| \geq 2 \\ \frac{1}{2}\left(\mathcal{J}_{i i}+\mathcal{J}_{j j}\right)+\mathcal{J}_{i j}+\mathcal{J}_{j i} & \text { if }|i-j|=1, \text { and } i \neq 0, j \neq N-1 \\ \mathcal{J}_{i j}+\mathcal{J}_{j i}+\frac{1}{2} \mathcal{J}_{00} & \text { if } i=0 \text { and } j=1 \\ \mathcal{J}_{i j}+\mathcal{J}_{j i}+\frac{1}{2} \mathcal{J}_{N-1 N-1} & \text { if } i=N-1 \text { and } j=N-2 .\end{cases}
$$

To keep the notation simple we do not make explicit the $X$ dependence in these objects.

By using (5.1) we obtain

$$
e^{-\lambda W_{T}}=\prod_{0 \leq i<j \leq N-1}\left(e^{-\lambda W_{\tau_{i}, \tau_{j}}}+1-1\right)=1+\sum_{\mathcal{R} \neq \emptyset} \prod_{\left(\tau_{i}, \tau_{j}\right) \in \mathcal{R}}\left(e^{-\lambda W_{\tau_{i}, \tau_{j}}}-1\right) .
$$

Here the summation is performed over all nonempty sets of different pairs of intervals, i.e. $\mathcal{R}=\left\{\left(\tau_{i}, \tau_{j}\right):\left(\tau_{i}, \tau_{j}\right) \neq\left(\tau_{i^{\prime}}, \tau_{j^{\prime}}\right)\right.$ whenever $\left.(i, j) \neq\left(i^{\prime}, j^{\prime}\right)\right\}$.

A break-up of the paths involves a corresponding factorization of the reference measure into Itô bridges for each subinterval. Put $X_{t_{k}}=x_{k}$ for the positions at the time-points of the division, $\forall k=0, \ldots, N$, with $-T=t_{0}<t_{1}<\ldots<t_{N}=T$. We write for a shorthand

$$
\nu_{T}^{\mathbf{x}}(\cdot) \equiv \nu_{T}\left(\cdot \mid X_{t_{0}}=x_{0}, \ldots, X_{t_{N}}=x_{N}\right)=\prod_{k=0}^{N-1} d \nu_{\tau_{k}}^{x_{k}, x_{k+1}}(\cdot) .
$$

Let $p_{t_{0}, \ldots, t_{N}}\left(x_{0}, \ldots, x_{N}\right)$ be the density with respect to $\prod_{k=0}^{N} d \omega\left(x_{k}\right), d \omega=\Psi^{2} d x$, of the joint distribution of positions of the path $X$ recorded at the time-points of the division. By Markovianness it follows that

$$
\begin{aligned}
p_{t_{0}, \ldots, t_{N}}\left(x_{0}, \ldots, x_{N}\right) & =\prod_{k=0}^{N-1} \pi_{b}\left(x_{k+1}, x_{k}\right)=\prod_{k=0}^{N-1}\left(\pi_{b}\left(x_{k+1}, x_{k}\right)-1+1\right) \\
& =1+\sum_{\mathcal{S} \neq \emptyset} \prod_{k: \tau_{k} \in \mathcal{S}}\left(\pi_{b}\left(x_{k+1}, x_{k}\right)-1\right),
\end{aligned}
$$

where $\pi_{t}$ is the transition kernel for the Itô diffusion given by (3.10). The summation runs over all nonempty sets $\mathcal{S}=\left\{\tau_{k}=\left(t_{k}, t_{k+1}\right)\right\}$ of different pairs of consecutive time-points.

In order to have a systematic control over these sums we introduce:

(1) Contours. Two distinct pairs of intervals $\left(\tau_{i}, \tau_{j}\right)$ and $\left(\tau_{i^{\prime}}, \tau_{j^{\prime}}\right)$ will be called directly connected and denoted $\left(\tau_{i}, \tau_{j}\right) \sim\left(\tau_{i^{\prime}}, \tau_{j^{\prime}}\right)$ if one interval of the pair $\left(\tau_{i}, \tau_{j}\right)$ coincides 
with one interval of the pair $\left(\tau_{i^{\prime}}, \tau_{j^{\prime}}\right)$. A set of connected pairs of intervals is a collection $\left\{\left(\tau_{i_{1}}, \tau_{j_{1}}\right), \ldots,\left(\tau_{i_{n}}, \tau_{j_{n}}\right)\right\}$ in which each pair of intervals is connected to another through a sequence of directly connected pairs, i.e., for any $\left(\tau_{i}, \tau_{j}\right) \neq\left(\tau_{i^{\prime}}, \tau_{j^{\prime}}\right)$ there exists $\left\{\left(\tau_{k_{1}}, \tau_{l_{1}}\right), \ldots,\left(\tau_{k_{m}}, \tau_{l_{m}}\right)\right\}$ such that $\left(\tau_{i}, \tau_{j}\right) \sim\left(\tau_{k_{1}}, \tau_{l_{1}}\right) \sim \ldots \sim\left(\tau_{k_{m}}, \tau_{l_{m}}\right) \sim$ $\left(\tau_{i^{\prime}}, \tau_{j^{\prime}}\right)$. A maximal set of connected pairs of intervals is called a contour, denoted by $\gamma$. We denote by $\bar{\gamma}$ the set of all intervals that are elements of the pairs of intervals belonging to contour $\gamma$, and by $\gamma^{*}$ the set of time-points of intervals appearing in $\bar{\gamma}$. Two contours $\gamma_{1}, \gamma_{2}$ are disjoint if they have no intervals in common, i.e. $\bar{\gamma}_{1} \cap \bar{\gamma}_{2}=\emptyset$. Clearly, $\mathcal{R}$ can be decomposed into sets of pairwise disjoint contours: $\mathcal{R}=\cup_{r \geq 1} \mathcal{R}_{r}$, where $\mathcal{R}_{r}=\left\{\gamma_{1}, \ldots, \gamma_{r}\right\}$ with $\bar{\gamma}_{i} \cap \bar{\gamma}_{j}=\emptyset, i \neq j ; i, j=1, \ldots, r$.

(2) Chains. A collection of consecutive intervals $\left\{\tau_{j}, \tau_{j+1} \ldots, \tau_{j+k}\right\}, j \geq 0, j+k \leq N-1$ is called a chain. As in the case of contours, $\bar{\varrho}$ and $\varrho^{*}$ mean the set of intervals belonging to the chain $\varrho$ and the set of time-points in $\varrho$, respectively. Two chains $\varrho_{1}, \varrho_{2}$ are called disjoint if they have no common time-points, i.e. $\varrho_{1}^{*} \cap \varrho_{2}^{*}=\emptyset$. Denote by $\partial^{-} \varrho$ resp. $\partial^{+} \varrho$ the leftmost resp. rightmost time-points belonging to $\varrho$.

(3) Clusters. Take a (non-ordered) set of disjoint contours and disjoint chains, $\Gamma=$ $\left\{\gamma_{1}, \ldots, \gamma_{r} ; \varrho_{1}, \ldots, \varrho_{s}\right\}$, with some $r \geq 1$ and $s \geq 0$. Note that such contours and chains may have common time-points. The notation $\Gamma^{*}=\left(\cup_{i} \gamma_{i}^{*}\right) \cup\left(\cup_{j} \varrho_{j}^{*}\right)$ means the set of all time-points appearing as beginnings or ends of intervals belonging to some contour or chain in $\Gamma$. Also, we put $\bar{\Gamma}=\left(\cup_{i} \bar{\gamma}_{i}\right) \cup\left(\cup_{j} \bar{\varrho}_{j}\right)$ for the set of intervals appearing in $\Gamma$ through entering some contours or chains. $\Gamma$ is called a cluster if $\Gamma^{*}$ is a connected collection of sets (in the usual sense), and for every $\varrho \in \Gamma$ we have that $\partial^{-} \varrho, \partial^{+} \varrho \in \cup_{j=1}^{r} \gamma_{j}^{*}$. This means that in a cluster chains have no loose ends. We denote by $\mathcal{K}_{N}$ the set of all clusters for a given $N$.

With these notations the sum in (5.2) is then further expanded as

$$
\sum_{\mathcal{R} \neq \emptyset} \prod_{\left(\tau_{i}, \tau_{j}\right) \in \mathcal{R}}\left(e^{-\lambda W_{\tau_{i}, \tau_{j}}}-1\right)=\sum_{r \geq 1} \sum_{\left\{\gamma_{1}, \ldots, \gamma_{r}\right\}} \prod_{k=1}^{r} \prod_{\left(\tau_{i}, \tau_{j}\right) \in \gamma_{k}}\left(e^{-\lambda W_{\tau_{i}, \tau_{j}}}-1\right)
$$

where now summation goes over collections $\left\{\gamma_{1}, \ldots, \gamma_{r}\right\}$ of contours such that $\bar{\gamma}_{k} \cap \bar{\gamma}_{k^{\prime}}=\emptyset$ unless $k=k^{\prime}$. In a similar way (5.3) appears in the form

$$
\sum_{\mathcal{S} \neq \emptyset} \prod_{k: \tau_{k} \in \mathcal{S}}\left(\pi_{b}\left(x_{k+1}, x_{k}\right)-1\right)=\sum_{s \geq 1} \sum_{\left\{\varrho_{1}, \ldots, \varrho_{s}\right\}} \prod_{j=1}^{s} \prod_{k: \tau_{k} \in \varrho_{j}}\left(\pi_{b}\left(x_{k+1}, x_{k}\right)-1\right) .
$$

Here $\left\{\varrho_{1}, \ldots, \varrho_{s}\right\}$ is a collection of disjoint chains, and this formula justifies how we defined them.

For every cluster $\Gamma=\left\{\gamma_{1}, \ldots, \gamma_{r} ; \varrho_{1}, \ldots, \varrho_{s}\right\} \in \mathcal{K}_{N}$ define the function

$$
\kappa_{\Gamma}=\prod_{l=1}^{r} \prod_{\left(\tau_{i}, \tau_{j}\right) \in \gamma_{l}}\left(e^{-\lambda W_{\tau_{i}, \tau_{j}}}-1\right) \prod_{m=1}^{s} \prod_{k: \tau_{k} \in \varrho_{m}}\left(\pi_{b}\left(x_{k+1}, x_{k}\right)-1\right) .
$$


Also, introduce the auxiliary probability measure

$$
d \chi_{N}(X)=\prod_{k=0}^{N-1} d \nu_{\tau_{k}}^{x_{k}, x_{k+1}}\left(X_{\tau_{k}}\right) \prod_{k=0}^{N} d \omega\left(x_{k}\right),
$$

and look at

$$
K_{\Gamma}=\mathbb{E}_{\chi}\left[\kappa_{\Gamma}\right],
$$

where $\chi$ is the unique extension over the real line of the family of consistent probabilities $\left\{\chi_{N}\right\}_{N \geq 1}$. Note that $\int\left(\pi_{b}\left(x_{k+1}, x_{k}\right)-1\right) d \omega\left(x_{k+1}\right)=\int\left(\pi_{b}\left(x_{k+1}, x_{k}\right)-1\right) d \omega\left(x_{k}\right)=0$. This is the reason why from a cluster we rule out chains having loose ends; for any such chain $\mathbb{E}_{\chi_{N}}\left[\kappa_{\Gamma}\right]=0$.

Define

$$
\phi^{T}\left(\Gamma_{1}, \ldots, \Gamma_{n}\right)= \begin{cases}1 & \text { if } n=1 \\ \sum_{G \in \mathcal{G}^{n}} \prod_{\{i, j\} \in G}\left(-1_{\Gamma_{i}^{*} \cap \Gamma_{j}^{*} \neq \emptyset}\right) & \text { if } n>1,\end{cases}
$$

with $\mathcal{G}^{n}$ the set of connected graphs on the vertex set $\{1, \ldots, n\}$. Note that $\phi^{T}\left(\Gamma_{1}, \ldots, \Gamma_{n}\right)=$ 0 if the graph on the vertex set $\{1, \ldots, n\}$ with edges $\{i, j\}$ drawn whenever $\Gamma_{i}^{*} \cup \Gamma_{j}^{*} \neq \emptyset$, is connected.

By putting (5.4), (5.3), (5.5), (5.6) and (5.8) together we obtain the cluster representation of the partition function $Z_{T}$.

Proposition 5.2 For every $T=N b / 2>0$ we have

$$
Z_{T}=1+\sum_{n \geq 1} \sum_{\substack{\left\{\Gamma_{1}, \ldots, \Gamma_{n}\right\} \in \mathcal{K}_{N} \\ \Gamma_{i}^{*} \cap \Gamma_{j}^{*}=\emptyset, i \neq j}} \prod_{l=1}^{n} K_{\Gamma_{l}}
$$

If the activities $K_{\Gamma}$ satisfy the bound

$$
\sum_{\substack{\Gamma \in \mathcal{K}_{N} \\ \Gamma^{*} \ni 0,|\bar{\Gamma}|=n}}\left|K_{\Gamma}\right| \leq c \eta^{n}
$$

for $\eta>0$ small enough, then the series above and at the right hand side of

$$
\log Z_{T}=\sum_{n \geq 1} \sum_{\substack{\left\{\Gamma_{1}, \ldots, \Gamma_{n}\right\} \in \mathcal{K}_{N} \\ 0 \in \Gamma_{1}^{*}}} \phi^{T}\left(\Gamma_{1}, \ldots, \Gamma_{n}\right) \prod_{l=1}^{n} K_{\Gamma_{l}}
$$

are absolutely convergent, uniformly in $N$, and the latter one gives the logarithm of the partition function for the interval $[-T, T]$.

The expression of the logarithm and the absolute convergence of the sums are a general result of cluster expansion techniques, for details of proof see [40]. 


\subsection{Convergence of cluster expansion}

Proposition 5.3 Suppose that there exist a function $D: \mathbb{N} \times \mathbb{N} \rightarrow(0, \infty)$ and numbers $\varepsilon, C>0$ with $\sup _{i \in \mathbb{N}} \sum_{j \in \mathbb{N}} D(i, j) \leq C$ such that for every $N>0$ and every cluster $\Gamma=\left\{\gamma_{1}, \ldots, \gamma_{r} ; \varrho_{1}, \ldots, \varrho_{s}\right\} \in \mathcal{K}_{N}$, the bound

$$
\left|K_{\Gamma}\right| \leq\left(\prod_{l=1}^{r} \prod_{\left(\tau_{i}, \tau_{j}\right) \in \gamma_{l}} \varepsilon D(i, j)\right) \varepsilon^{\sum_{m=1}^{s}\left|\bar{\varrho}_{m}\right|}
$$

holds. Then there is a constant $c>0$ and a function $0<\eta(\varepsilon)<1$ with $\eta \rightarrow 0$ as $\varepsilon \rightarrow 0$ such that

$$
\sum_{\substack{\Gamma \in \mathcal{K}_{N} \\ \Gamma^{*} \ni 0,|\bar{\Gamma}|=n}}\left|K_{\Gamma}\right| \leq c \eta^{n}
$$

The function $D(i, j)$ will be specified in Proposition 5.5 below.

Proof. We put for a shorthand $\mathcal{D}(\gamma)=\prod_{\left(\tau_{i}, \tau_{j}\right) \in \gamma} \varepsilon D(i, j)$. Consider the function of complex variable $z$

$$
H(z ; \varepsilon)=\sum_{\Gamma \in \mathcal{K}_{N}: \Gamma^{*} \ni 0} K_{\Gamma} z^{|\bar{\Gamma}|}=\sum_{\substack{\Gamma \in \mathcal{K}_{N}: \Gamma^{*} \ni 0 \\ \Gamma \supset \text { one contour }}} K_{\Gamma} z^{|\bar{\Gamma}|}+\sum_{\substack{\Gamma \in \mathcal{K}_{N}: \Gamma^{*} \ni 0 \\ \Gamma \supset \text { more than one contour }}} K_{\Gamma} z^{|\bar{\Gamma}|}
$$

We show that for sufficiently small $\varepsilon>0$ this is an analytic function of $z$ in a circle of radius $R(\varepsilon)$ which diverges as $\varepsilon \rightarrow 0$. Moreover, we show that within this circle $H(z ; \varepsilon)$ is uniformly bounded in $\varepsilon$. This will then imply (5.13) by choosing $\eta(\varepsilon)=1 / R(\varepsilon)$.

We start by estimating the second sum; the first is simpler as it involves clusters having a single contour. Our strategy is first to bound it by a sum taken over graphs whose vertices are the contours of the same cluster. The sums over graphs will then be bounded by sums taken over trees.

Bounds by sums over graphs For each $r \geq 2$ consider in $\mathcal{K}_{N}$ those clusters $\Gamma$ that have $r$ contours. For given $\Gamma \in \mathcal{K}_{N}$ let $\mathcal{V}_{r}=\left\{\gamma_{1}, \ldots, \gamma_{r}\right\}$ be the collection of these contours. We construct connected graphs $G$ by drawing edges between the elements of $\mathcal{V}_{r}$ considered as vertex set. Connected graphs are those for which either $\gamma_{i}^{*} \cap \gamma_{j}^{*} \neq \emptyset$ or there exists $\varrho_{l} \in \Gamma$ such that $\varrho_{l}^{*} \cap \gamma_{i}^{*} \neq \emptyset \neq \varrho_{l}^{*} \cap \gamma_{j}^{*}$. Let $\mathcal{G}_{r}$ denote the set of all possible such graphs.

Consider the collection of reduced chains $\left\{\hat{\varrho}_{1}, \ldots, \hat{\varrho}_{s}\right\}$ with the properties:

(1) for every pair $\left\{\gamma_{i}, \gamma_{j}\right\} \in G \in \mathcal{G}_{r}, \gamma_{i}^{*} \cap \gamma_{j}^{*}=\emptyset$, there is at least one chain $\hat{\varrho}_{l}$ of this collection connecting $\gamma_{i}$ and $\gamma_{j}$ (i.e. $\bar{\varrho}_{l} \cap \bar{\gamma}_{i}=\emptyset=\overline{\hat{\varrho}}_{l} \cap \bar{\gamma}_{j}$ and $\hat{\varrho}_{l}^{*} \cap \gamma_{i}^{*} \neq \emptyset \neq \hat{\varrho}_{l}^{*} \cap \gamma_{j}^{*}$ ), and for any pair $\left\{\gamma_{i}, \gamma_{j}\right\} \notin G$ such a chain does not occur;

(2) $\cup_{i} \bar{\gamma}_{i} \cap \cup_{j} \overline{\hat{\varrho}}_{j}=\emptyset$ and $\cup_{j}\left\{\partial^{-} \hat{\varrho}_{j}, \partial^{+} \hat{\varrho}_{j}\right\} \subset \cup_{i} \gamma_{i}^{*}$;

(3) $0 \in\left(\cup_{i} \gamma_{i}^{*}\right) \cup\left(\cup_{j} \hat{\varrho}_{j}^{*}\right)$;

(4) each chain $\hat{\varrho}_{k}$ connects a pair $\left\{\gamma_{i}, \gamma_{j}\right\} \in G \in \mathcal{G}_{r}$ or fills a gap within a contour $\gamma_{i}$. 
We call a collection of reduced chains compatible with graph $G$ if it satisfies the conditions above and denote it $\left\{\hat{\varrho}_{1}, \ldots, \varrho_{s}\right\}_{G}$. Note that each $\varrho$ can join only one pair of contours. A collection of reduced chains is then constructed through the following steps:

(1) first remove all chains $\varrho_{l} \in \Gamma$ for which $\bar{\varrho}_{l} \subset \cup_{i=1}^{r} \bar{\gamma}_{i}$;

(2) for all remaining chains $\varrho_{k} \in \Gamma, \bar{\varrho}_{k} \not \subset \cup_{i=1}^{r} \bar{\gamma}_{i}$ remove all intervals from the set $\bar{\varrho}_{k} \cap\left(\cup_{i=1}^{r} \bar{\gamma}_{i}\right)$;

(3) of the remaining intervals form all possible collections of non-empty chains denoted by $\left\{\hat{\varrho}_{1}, \ldots, \hat{\varrho}_{s}\right\}$.

Then by Proposition 5.32 we write

$$
\begin{aligned}
& \left|\sum_{\substack{\Gamma: \Gamma^{*} \ni 0 \\
\Gamma \supset \text { more than one contour }}} K_{\Gamma} z^{|\bar{\Gamma}|}\right| \leq \\
& \quad \sum_{r \geq 2} \sum_{\left\{\gamma_{1}, \ldots, \gamma_{r}\right\}} \sum_{G \in \mathcal{G}_{r}} \sum_{s \geq 0} \prod_{i=1}^{r}\left((|z|(1+\varepsilon|z|))^{\left|\overline{\gamma_{i}}\right|} \mathcal{D}\left(\gamma_{i}\right) \sum_{\substack{\left\{\hat{\rho}_{1}, \ldots, \hat{Q}_{s}\right\}_{G} \\
0 \in \cup_{i} \gamma_{i}^{*} \cup \hat{\varrho}_{i}^{*}}} \prod_{j=1}^{s}(\varepsilon|z|)^{\left|\hat{\varrho}_{j}\right|} .\right.
\end{aligned}
$$

Note that for fixed $\left\{\gamma_{1}, \ldots, \gamma_{r}\right\}$ the collection $\left\{\hat{\varrho}_{1}, \ldots, \varrho_{m}\right\}$ can be obtained from many possible collections of chains $\left\{\varrho_{1}, \ldots, \varrho_{s}\right\}$. This gives the factor $(1+\varepsilon|z|)^{\left|\bar{\gamma}_{i}\right|}$ appearing at the right hand side of (5.15). From now on we assume that $\varepsilon|z| \leq 1$ so that we can estimate this factor by $2^{\left|\bar{\gamma}_{i}\right|}$.

Now consider the last sum above involving the reduced chains. In this sum, either 0 belongs to a contour or some chains. In the second case there is a factor of $(\varepsilon|z|)^{\operatorname{dist}(0,\{\gamma\})}$ appearing in the sum, so we can estimate the sum by

$$
(\varepsilon|z|)^{\operatorname{dist}(0, \gamma) / 2} \sum_{\substack{\left\{\hat{\varrho}_{1}, \ldots, \hat{\varrho}_{s}\right\}_{G} \\ 0 \in \cup_{i} \gamma_{i}^{*} \cup \hat{\varrho}_{i}^{*}}} \prod_{j=1}^{s}(\varepsilon|z|)^{\left|\overline{\hat{\varrho}_{j}}\right| / 2} \leq \sum_{k}(\varepsilon|z|)^{\operatorname{dist}\left(0, \gamma_{k}\right) / 2} \sum_{\substack{\left\{\hat{\varrho}_{1}, \ldots, \hat{\rho}_{s}\right\}_{G} \\ 0 \in \cup_{i} \gamma_{i}^{*} \cup \hat{\varrho}_{i}^{*}}}^{s} \prod_{j=1}^{s}(\varepsilon|z|)^{\left|\hat{\varrho}_{j}\right| / 2} .
$$

For each contour $\gamma_{i}$ the sum over all reduced chains belonging to this contour cannot be larger than $2^{\left|\overline{\gamma_{i}}\right|}$ since the number of such chains is bounded by $\overline{\gamma_{i}}$ (when every chain separates each two successive intervals in the contour). Moreover for each couple of contours $\left(\gamma_{i}, \gamma_{j}\right)$ the contribution to the sum of the chains connecting them is given by

$$
2^{\left|\overline{\gamma_{i}}\right|+\left|\overline{\gamma_{j}}\right|}(\varepsilon|z|)^{\operatorname{dist}\left(\gamma_{i}, \gamma_{j}\right) / 2}
$$

since there is at least one chain longer than $\operatorname{dist}\left(\gamma_{i}, \gamma_{j}\right)$ and the rest of the chains contribute into the combinatorial prefactor. This gives

$$
\begin{aligned}
& \quad \sum_{\substack{\Gamma: \Gamma^{*} \ni 0 \\
\Gamma \supset \text { more than one contour }}} K_{\Gamma} z^{|\bar{\Gamma}|} \mid \leq \\
& \quad \sum_{r \geq 2} \sum_{\left\{\gamma_{1}, \ldots, \gamma_{r}\right\}} \sum_{k} \prod_{i=1}^{r}(8|z|)^{\left|\bar{\gamma}_{i}\right|} \mathcal{D}\left(\gamma_{i}\right)(\varepsilon|z|)^{\operatorname{dist}\left(0, \gamma_{k}\right) / 2} \sum_{G \in \mathcal{G}_{r}} \prod_{\left\{\gamma_{i}, \gamma_{j}\right\} \in G}(\varepsilon|z|)^{\operatorname{dist}\left(\gamma_{i}, \gamma_{j}\right) / 2} .
\end{aligned}
$$


Bounds by sums over trees We use the tree-graph bound (cf. Lemma 8, Ch. 2, Sect. 4 of [40]) to get

$$
\sum_{G \in \mathcal{G}_{r}} \prod_{\left\{\gamma_{i}, \gamma_{j}\right\} \in G}(\varepsilon|z|)^{\operatorname{dist}\left(\gamma_{i}, \gamma_{j}\right) / 2} \leq \prod_{i, j=1}^{r}\left(1+(\varepsilon|z|)^{\operatorname{dist}\left(\gamma_{i}, \gamma_{j}\right) / 2}\right) \sum_{T \in \mathcal{T}_{r}} \prod_{\left\{\gamma_{i}, \gamma_{j}\right\} \in T}(\varepsilon|z|)^{\operatorname{dist}\left(\gamma_{i}, \gamma_{j}\right) / 2}
$$

where $\mathcal{T}_{r}$ is the set of trees on the vertex set $\left\{\gamma_{1}, \ldots, \gamma_{r}\right\}$. Moreover we have

$$
\prod_{i, j=1}^{r}\left(1+(\varepsilon|z|)^{\operatorname{dist}\left(\gamma_{i}, \gamma_{j}\right) / 2}\right) \leq 2^{2 r} \leq 2^{2 \sum_{i}\left|\bar{\gamma}_{i}\right|}
$$

thus (5.15) is further estimated by

$$
\begin{aligned}
& \sum_{\substack{\Gamma: \Gamma^{*} \ni 0 \\
\Gamma \supset \text { more than one contour }}} K_{\Gamma} z^{|\bar{\Gamma}|} \mid \leq \\
& \quad \sum_{r \geq 2} \sum_{\left\{\gamma_{1}, \ldots, \gamma_{r}\right\}} \sum_{k} \prod_{i=1}^{r}(32|z|)^{\left|\bar{\gamma}_{i}\right|} \mathcal{D}\left(\gamma_{i}\right)(\varepsilon|z|)^{\operatorname{dist}\left(0, \gamma_{k}\right) / 2} \sum_{T \in \mathcal{T}_{r}} \prod_{\left\{\gamma_{i}, \gamma_{j}\right\} \in G}(\varepsilon|z|)^{\operatorname{dist}\left(\gamma_{i}, \gamma_{j}\right) / 2} .
\end{aligned}
$$

Take the trees over vertex set $\{1, \ldots, r\}$ obtained through $\gamma_{k} \mapsto k, \forall k=1, \ldots, r$; denote them $\tilde{T}$ and the set of all such trees by $\tilde{\mathcal{T}}_{r}$. Then we re-sum in (5.18):

$$
\begin{aligned}
& \text { r.h.s. (15.18) } \leq \\
& \qquad \sum_{r=2}^{\infty} \frac{1}{r !} \sum_{\tilde{T} \in \tilde{T}_{r}} \sum_{i^{*}=1}^{r} \sum_{\left(\gamma_{1}, \ldots, \gamma_{r}\right)} \prod_{i=1}^{r}(32|z|)^{\left|\bar{\gamma}_{i}\right|}(\varepsilon|z|)^{\operatorname{dist}\left(0, \gamma_{i}^{*}\right) / 2} \mathcal{D}\left(\gamma_{i}\right) \prod_{\{i, j\} \in \tilde{T}}(\varepsilon|z|)^{\operatorname{dist}\left(\gamma_{i}, \gamma_{j}\right) / 2} .
\end{aligned}
$$

The change of bracket indicates that the third sum here is performed over all ordered collections of disjoint contours. Fix an enumeration of $\tilde{T}$ and pick its first element $i_{0}$. We estimate first

$$
\sum_{\left(\gamma_{1}, \ldots, \gamma_{r}\right)} \prod_{i=1}^{r}(32|z|)^{\left|\bar{\gamma}_{i}\right|} \mathcal{D}\left(\gamma_{i}\right) \prod_{\{i, j\} \in \tilde{T}}(\varepsilon|z|)^{\operatorname{dist}\left(\gamma_{i}, \gamma_{j}\right) / 2} .
$$

Let $j_{0} \neq i_{0}$ be an end vertex of tree $\tilde{T}$ being joint only with vertex $k_{0}$. Then

$$
\begin{aligned}
\sum_{\gamma_{j_{0}}}(32|z|)^{\left|\bar{\gamma}_{j_{0}}\right|} & \mathcal{D}\left(\gamma_{j_{0}}\right)(\varepsilon|z|)^{\operatorname{dist}\left(\gamma_{k_{0}}, \gamma_{j_{0}}\right) / 2} \\
& \leq \sum_{\tau^{\prime \prime} \in \bar{\gamma}_{k_{0}}} \sum_{\gamma_{j_{0}}} \sum_{\tau^{\prime} \in \bar{\gamma}_{j_{0}}}(\varepsilon|z|)^{\operatorname{dist}\left(\tau^{\prime}, \tau^{\prime \prime}\right) / 2}(32|z|)^{\left|\bar{\gamma}_{j_{0}}\right|} \mathcal{D}\left(\gamma_{j_{0}}\right) \\
& \leq \sum_{\tau^{\prime \prime} \in \bar{\gamma}_{k_{0}}} \sum_{\tau^{\prime}}(\varepsilon|z|)^{\operatorname{dist}\left(\tau^{\prime}, \tau^{\prime \prime}\right) / 2} \sum_{\gamma_{j_{0}}: \tau^{\prime} \in \bar{\gamma}_{j_{0}}}(32|z|)^{\left|\bar{\gamma}_{j_{0}}\right|} \mathcal{D}\left(\gamma_{j_{0}}\right) .
\end{aligned}
$$


Here we used that $(\varepsilon|z|)^{\operatorname{dist}\left(\gamma, \gamma^{\prime}\right) / 2} \leq \sum_{\tau \in \gamma, \tau^{\prime} \in \gamma^{\prime}}(\varepsilon|z|)^{\operatorname{dist}\left(\tau, \tau^{\prime}\right) / 2}$. By using Lemma 5.4 below and the bound

$$
\sum_{k=2}^{\infty} a^{k-1} k^{m} \leq \frac{2^{m} m ! e a}{1-e a}
$$

obtained via complex integration, we further estimate (5.21) by

$$
\sum_{\tau^{\prime \prime} \in \bar{\gamma}_{k_{0}}} \sum_{\tau^{\prime}}(\varepsilon|z|)^{\operatorname{dist}\left(\tau^{\prime}, \tau^{\prime \prime}\right) / 2} \sum_{k=2}^{\infty}(32|z|)^{k}(C \varepsilon)^{k-1} \leq \frac{64 C\left|\bar{\gamma}_{k_{0}}\right| \varepsilon|z|}{\left(1-(\varepsilon|z|)^{1 / 2}\right)(1-32 C \varepsilon|z|)} .
$$

From now on we choose $z$ such that $32 C \varepsilon|z|<1$ holds.

Next we go on by taking the next vertex of $\tilde{\mathcal{T}}$ in line, say $j_{1} \neq i_{0}$ connecting with $k_{1}$. We iterate the procedure for the new tree obtained by deleting from $\tilde{T}$ the vertex $j_{0}$ and edge $\left(j_{0}, k_{0}\right)$. If $j_{1} \neq k_{0}$, we get again an estimate of the type (5.23). If $j_{1}=k_{0}$, we estimate

$$
\sum_{\tau^{\prime \prime} \in \gamma_{k_{1}}} \sum_{\tau^{\prime}}(\varepsilon|z|)^{\operatorname{dist}\left(\tau^{\prime}, \tau^{\prime \prime}\right) / 2} \sum_{\gamma_{j_{1}}: \tau^{\prime} \in \gamma}\left|\bar{\gamma}_{j_{1}}\right|(32|z|)^{\left|\bar{\gamma}_{j_{1}}\right|} \mathcal{D}(\gamma) \leq G_{1}\left|\bar{\gamma}_{k_{1}}\right| \sum_{k=2}^{\infty}(32|z|)^{k} k(C \varepsilon)^{k-1},
$$

with some $C_{1}>0$. Continuing this procedure inductively we get after summation over $\gamma_{j_{m}}, j_{m} \neq i_{0}$, connected to $\gamma_{k_{m}}$, the net contribution

$$
\begin{aligned}
C_{1}\left|\bar{\gamma}_{k_{m}}\right| \sum_{k=1}^{\infty}(32|z|)^{k} k^{l_{j_{m}}-1}(C \varepsilon)^{k-1} & \leq G_{1}\left|\gamma_{k_{m}}\right| \sum_{k=2}^{\infty}\left(C_{2}|z|\right)^{k} k^{l_{j_{m}}-1}(C \varepsilon)^{k-1} \\
& \leq C_{[5}\left|\gamma_{k_{m}}\right||z| \sum_{k=2}^{\infty}\left(C_{4} \varepsilon|z|\right)^{k-1} k^{l_{j_{1}}-1}
\end{aligned}
$$

where $l_{j_{m}}$ is the degree of vertex $j_{m}$, i.e. the number of edges of $\tilde{T}$ incident to $j_{m}$, and $C_{2}, C_{3}>0, C_{4}=C_{9}, C_{5}=C_{2} C_{3}$ is the long sequence of constants. By using (5.22) again, we estimate (5.20) further for fixed $\gamma_{i_{0}}$ and $\tilde{T}$ to get

$$
\sum_{\substack{\gamma_{k}: k \neq i_{0} \\ k=1, \ldots, r}} \prod_{\{i, j\} \in \tilde{T}}(\varepsilon|z|)^{\operatorname{dist}\left(\tau_{i}, \tau_{j}\right) / 2} \prod_{i \neq i_{0}} \mathcal{D}\left(\gamma_{i}\right)(32|z|)^{\left|\bar{\gamma}_{i}\right|} \leq\left|\bar{\gamma}_{i_{0}}\right|^{l_{i_{0}}} \prod_{k \neq i_{0}} 2^{l_{j_{k}}}\left(l_{j_{k}}-1\right) !\left(k_{3} \varepsilon|z|^{2}\right)^{r-1}
$$

where we used that $\varepsilon|z| \leq 1 /\left(C_{4}^{e}\right)$. Thus we need furthermore (see (5.19))

$$
\begin{aligned}
& \sum_{\gamma_{i_{0}}}\left|\bar{\gamma}_{i_{0}}\right|^{l_{i_{0}}} \mathcal{D}\left(\gamma_{i_{0}}\right)(16|z|(1+\varepsilon|z|))^{\left|\gamma_{i_{0}}\right|}(\varepsilon|z|)^{\operatorname{dist}\left(0, \gamma_{i_{0}}\right) / 2} \\
& \quad \leq \sum_{\tau}(\varepsilon|z|)^{\operatorname{dist}(0, \tau) / 2} \sum_{\gamma_{i_{0}}: \tau \in \gamma_{i_{0}}}\left|\bar{\gamma}_{i_{0}}\right|^{l_{i_{0}}} \mathcal{D}\left(\gamma_{i_{0}}\right)(32|z|)^{\left|\gamma_{i_{0}}\right|} .
\end{aligned}
$$

By a repetition of the arguments above we get

$$
\begin{aligned}
\sum_{\gamma_{i_{0}}: \tau \in \gamma_{i_{0}}}\left|\bar{\gamma}_{i_{0}}\right|^{l_{i_{0}}} \mathcal{D}\left(\gamma_{i_{0}}\right)(32|z|)^{\left|\gamma_{i_{0}}\right|} & \leq \sum_{k=2}^{\infty} k^{l_{i_{0}}}(C \varepsilon)^{k-1}(32|z|)^{k} \\
& \leq C_{6} 2^{l_{i_{0}}} l_{i_{0}} ! \varepsilon|z|^{2},
\end{aligned}
$$


with $C_{6}>0$. Summation over $\tau$ gives $\sum_{\tau}(\varepsilon|z|)^{\operatorname{dist}(0, \tau) / 2} \leq C_{7}$, with some $C_{7}>0$, hence we finally obtain for fixed $\tilde{T}$ and $i_{0}$

$$
2^{l_{i_{0}}} l_{i_{0}} ! \prod_{j_{k} \neq i_{0}} 2^{l_{k}}\left(l_{j_{k}}-1\right) ! G_{7}\left(C_{6_{6}}^{\varepsilon|z|^{2}}\right)^{r-1} \leq G_{7}\left(2^{2} C_{[6} \varepsilon|z|^{2}\right)^{r-1} \prod_{k=1}^{r} l_{j_{k}} !
$$

where we used the fact $\sum_{k=1}^{r} l_{j_{k}}=2(r-1)$ for trees. An upper bound on the number of trees with vertices $\{1, \ldots, r\}$ and degrees $\left\{l_{1}, \ldots, l_{r}\right\}$ is [40]

$$
\frac{2^{r-2}(r-2) !}{\prod_{j=1}^{r} l_{j} !}
$$

Moreover, the number of collections $\left\{l_{1}, \ldots, l_{r}\right\}$ such that $l_{i}>0$ and $\sum_{i} l_{i}=2(r-1)$ is bounded from above by $2^{2(r-1)}$. Hence, by summing over $i_{0}$ and combining this estimate with (5.24), we get

$$
\sum_{\substack{\Gamma: \Gamma^{*} \ni 0 \\ \Gamma \supset \text { more than one contour }}} K_{\Gamma}|z|^{|\bar{\Gamma}|} \leq c \varepsilon|z|^{2}
$$

with some constant $c>0$. This completes the estimate of the second term in (5.14). The first term there can be handled in a similar way with substantial simplifications due to the fact that only one contour occurs in the clusters.

It is seen then that by choosing $z$ such that $\varepsilon|z|^{2} \leq$ const, the $\operatorname{sum} \sum_{\Gamma} K_{\Gamma} z^{|\bar{\Gamma}|}$ converges and is bounded. Hence $H(z)$ is an analytic function within a circle of radius $R(\varepsilon)$ with $R(\varepsilon) \rightarrow \infty$ as $\varepsilon \rightarrow 0$, and is bounded by a constant independent of $\varepsilon$. Thus

$$
\sum_{\substack{\Gamma: \Gamma^{*} \ni 0 \\|\Gamma|=n}}\left|K_{\Gamma}\right| \leq \text { const } R(\varepsilon)^{-n}:=\text { const } \eta(\varepsilon)^{n}
$$

with suitable constants.

Finally we show the lemma referred to in the proof above.

Lemma 5.4 There is a constant $C>0$ such that for any interval $\tau$ and integer $k \geq 2$

$$
\sum_{\substack{\gamma: \bar{\gamma} \ni \tau \\|\hat{\gamma}|=k}} \mathcal{D}(\gamma) \leq(C \varepsilon)^{k-1}
$$

ProOF.

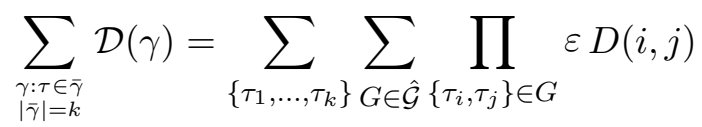

Here $\hat{\mathcal{G}}$ denotes the set of connected graphs with vertices $\tau_{1}, \ldots, \tau_{k}$. Note that for fixed $i_{0}$ we have $\sum_{\tau_{j}: j \neq i_{0}} \varepsilon D\left(i_{0}, j\right) \leq C \varepsilon$ with some $C>0$. Thus by using (15.17) we find

$$
\sum_{G \in \hat{\mathcal{G}}\left\{\tau_{i}, \tau_{j}\right\} \in G} \varepsilon D(i, j) \leq e^{k C \varepsilon} \sum_{T \in \hat{\mathcal{T}}} \prod_{\left\{\tau_{i}, \tau_{j}\right\} \in T} \varepsilon D(i, j)
$$


where $\hat{\mathcal{T}}$ are trees for the same vertex set as for $\hat{\mathcal{G}}$. Next order the collection $\left\{\tau_{1}, \ldots, \tau_{k}\right\}$ further to get

$$
\sum_{\left\{\tau_{1}, \ldots, \tau_{k}\right\}} \sum_{T \in \hat{T}} \prod_{\{i, j\} \in \tilde{\mathcal{T}}} \varepsilon D(i, j)=\frac{1}{k !} \sum_{\left(\tau_{1}, \ldots, \tau_{k}\right)} \sum_{\tilde{T} \in \tilde{\mathcal{T}}} \prod_{\{i, j\} \in \tilde{T}} \varepsilon D(i, j),
$$

with the same $\tilde{\mathcal{T}}$ as previously. We then obtain inductively

$$
\sum_{\left(\tau_{1}, \ldots, \tau_{k}\right)} \prod_{\left(\tau_{i}, \tau_{j}\right) \in \mathcal{T}} \varepsilon D(i, j) \leq(C \varepsilon)^{k-1}
$$

Since the number of trees having $k$ vertices is $k^{k-2}$ [40], by using Stirling's formula, (5.29) and (5.30) we complete the proof of the lemma.

\subsection{Cluster estimates}

Having the abstract cluster expansion at hand, we turn now to establishing the bounds (5.12) on the cluster activities.

Proposition 5.5 There exists $\delta>1$ and a function $\varepsilon(\lambda)<\infty$ with $\varepsilon(\lambda) \rightarrow 0$ as $\lambda \rightarrow 0$ and $b=b(\lambda) \geq 1$ such that for every $N>0$ and every cluster $\Gamma=\left\{\gamma_{1}, \ldots, \gamma_{r} ; \varrho_{1}, \ldots, \varrho_{s}\right\} \in \mathcal{K}_{N}$, the bound

$$
\left|K_{\Gamma}\right| \leq \prod_{l=1}^{r} \prod_{\left(\tau_{i}, \tau_{j}\right) \in \gamma_{l}} \frac{\varepsilon}{(1+b|i-j-1|)^{\delta}} \prod_{m=1}^{s} \varepsilon^{\left|\bar{\varrho}_{m}\right|}
$$

holds.

The first product (over the contours) above is our $D(i, j)$ in Proposition 5.3 above, and it is readily seen that it satisfies the condition given there.

Proof. By Hölder inequality

$$
\begin{aligned}
\left|K_{\Gamma}\right| \leq & \prod_{l=1}^{r} \prod_{\left(\tau_{i}, \tau_{j}\right) \in \gamma_{l}}\left(\int \mathbb{E}_{\nu_{T}^{\mathbf{x}}}\left[\left|e^{-\lambda W_{\tau_{i}, \tau_{j}}}-1\right|^{n_{i j}}\right] d \omega^{\otimes N}(\mathbf{x})\right)^{1 / n_{i j}} \times \\
& \times \prod_{m=1}^{s} \prod_{k: \tau_{k} \in \varrho_{m}}\left(\int\left|\pi_{b}\left(x_{k+1}, x_{k}\right)-1\right|^{\beta} d \omega\left(x_{k}\right) d \omega\left(x_{k+1}\right)\right)^{1 / \beta}
\end{aligned}
$$

with suitable exponents. We choose $\beta=4, n_{i j}=A|i-j+1|^{\Delta}$, with $\Delta>1$ to be specified below. Taken with correct multiplicities, we pick $A$ such that

$$
\frac{2}{\beta}+\sum_{\substack{j \in \mathbb{N} \\ j \geq i}} \frac{2}{n_{i j}}=\frac{1}{2}+\frac{2}{A} \sum_{k=1}^{\infty} \frac{1}{k^{\Delta}} \leq 1 .
$$

The first part of estimate (5.32) follows by Lemma 5.7, the second by Lemma 5.6 below. By choosing $b=-\log |\lambda| /(\Lambda+C)$ with suitable $C>0$, we have $\lambda e^{C b}=e^{-\Lambda b}=|\lambda|^{\Lambda /(\Lambda+C)}=$ : $\varepsilon$, thus the estimate (5.32) is finally obtained. 
Lemma 5.6 For large enough $b>0$ there is a constant $C>0$ such that

$$
\left|\pi_{b}(x, y)-1\right| \leq C e^{-\Lambda b},
$$

uniformly in $x, y \in \mathbb{R}^{d}$, where $\Lambda>0$ is the spectral gap of the Schrödinger operator $H=H_{0}+V$.

Proof. By assumption the potential is chosen so that $H$ is intrinsically ultracontractive, i.e., for each $b>0, C_{b}=\left\|\pi_{b}\right\|_{L^{\infty}\left(\mathbb{R}^{2 d}\right)}<\infty$. By the semigroup property of $\pi_{b}$ and the fact that $\int \pi_{b}(x, y) d \omega(y)=1$ for each $x$, for $b>2$ we have

$$
\begin{aligned}
\left|\pi_{b}(x, y)-1\right| & =\left|\int d \xi \int d \eta \pi_{1}(x, \xi) \Psi^{2}(\xi)\left(\pi_{b-2}(\xi, \eta)-1\right) \Psi^{2}(\eta) \pi_{1}(\eta, y)\right| \\
& \leq C_{1}^{2} \int d \xi \int d \eta \Psi(\xi)\left|\hat{\pi}_{b-2}(\xi, \eta)-\Psi(\xi) \Psi(\eta)\right| \Psi(\eta) \\
& \leq C_{1}^{2}\left(\int d \xi \int d \eta\left(\hat{\pi}_{b-2}(\xi, \eta)-\Psi(\xi) \Psi(\eta)\right)^{2}\right)^{1 / 2} \\
& =C_{1}^{2} e^{-(b-2)\left(E_{1}-E\right)}\left(\sum_{k \geq 2} e^{-2(b-2)\left(E_{k}-E_{1}\right)}\right)^{1 / 2} \leq C e^{-\Lambda b}
\end{aligned}
$$

where $E=\inf \operatorname{Spec} H$. The last but first step comes about as follows. $\left\|\pi_{b}\right\|_{L^{\infty}\left(\mathbb{R}^{2 d}\right)}<\infty$ implies that $\hat{\pi}_{b} \in L^{2}\left(\mathbb{R}^{2 d}, d x\right)$ for each $b>0$. Thus $e^{-b H}$ is a Hilbert-Schmidt operator for each $b>0$, in particular $H$ has a purely discrete spectrum with eigenvalues $E<E_{1} \leq$ $E_{2} \leq \ldots$ With $P_{\Psi}$, the projection onto the subspace of $L^{2}\left(\mathbb{R}^{d}, d x\right)$ spanned by $\Psi$, the last equality gives the Hilbert-Schmidt norm of $e^{-(b-2) H}-P_{\Psi}$.

Lemma 5.7 Assume that $\beta>3$. Then there exists $\delta>1$ and constants $C_{8}, C_{9}<\infty$ such that, for $\lambda$ small enough

$$
\left(\int \mathbb{E}_{\nu_{T}^{\mathbf{x}}}\left[\left|e^{\lambda W_{\tau_{i}, \tau_{j}}}-1\right|^{n_{i j}}\right] d \omega^{\otimes N}(\mathbf{x})\right)^{1 / n_{i j}} \leq q \underline{8} \lambda e^{C b}(1+b|i-j-1|)^{\delta}
$$

for all $i, j$ and $b \geq 1$.

Proof. Note that the conditional expectation $\mathbb{E}_{\nu_{T}^{\mathbf{x}}}\left[\left|e^{\lambda W_{\tau_{i}, \tau_{j}}}-1\right|^{n_{i j}}\right]$ depends only on $x_{i}, x_{i+1}, x_{j}, x_{j+1}$. Thus we can write with a slight abuse of notation,

$$
\int \mathbb{E}_{\nu_{T}^{\mathbf{x}}}\left[\left|e^{\lambda W_{\tau_{i}, \tau_{j}}}-1\right|^{n_{i j}}\right] d \omega^{\otimes N}(\mathbf{x})=\int \mathbb{E}_{\nu_{T}^{\mathbf{x}}}\left[\left|e^{\lambda W_{\tau_{i}, \tau_{j}}}-1\right|^{n_{i j}}\right] d \omega^{\otimes 4}\left(x_{i}, x_{i+1}, x_{j}, x_{j+1}\right)
$$


when $|i-j|>1$, otherwise one integral must be ignored in this expression. Then by (3.12) the problem reduces to estimates on the multiple Brownian bridge $\widehat{\mathcal{W}_{T}^{\mathrm{x}}}$ :

$$
\begin{aligned}
& A=\int \mathbb{E}_{\nu_{T}^{\mathbf{x}}}\left[\left|e^{\lambda W_{\tau_{i}, \tau_{j}}}-1\right|^{n_{i j}}\right] d \omega^{\otimes 4}\left(x_{i}, x_{i+1}, x_{j}, x_{j+1}\right) \\
& \leq \int \frac{\mathbb{E}_{\widehat{\mathcal{W}_{T}^{\mathrm{x}}}}\left[\left|e^{\lambda W_{\tau_{i}, \tau_{j}}}-1\right|^{n_{i j}} e^{-V_{\tau_{i}}-V_{\tau_{j}}}\right]}{Z_{b} \Psi\left(x_{i}\right) \Psi\left(x_{i+1}\right) \pi_{b}\left(x_{i}, x_{i+1}\right)} \frac{\Pi_{b}\left(x_{i}, x_{i+1}\right) \Pi_{b}\left(x_{j}, x_{j+1}\right)}{Z_{b} \Psi\left(x_{j}\right) \Psi\left(x_{j+1}\right) \pi_{b}\left(x_{j}, x_{j+1}\right)} d \omega^{\otimes 4}\left(x_{i}, x_{i+1}, x_{j}, x_{j+1}\right) \\
& \leq C Z_{b}^{-2} \int \mathbb{E}_{\widehat{\mathcal{W}_{T}^{\mathrm{x}}}}\left[\left|e^{\lambda W_{\tau_{i}, \tau_{j}}}-1\right|^{n_{i j}} e^{-V_{\tau_{i}}-V_{\tau_{j}}}\right] \Pi_{b}\left(x_{i}, x_{i+1}\right) \Pi_{b}\left(x_{j}, x_{j+1}\right) \times \\
& \times \Psi\left(x_{i}\right) \Psi\left(x_{i+1}\right) \Psi\left(x_{j}\right) \Psi\left(x_{j+1}\right) d x_{i} d x_{i+1} d x_{j} d x_{j+1},
\end{aligned}
$$

where we used Lemma 5.6 and chose $b$ large enough so that $\sup _{x, y}\left|\pi_{b}(x, y)-1\right| \leq C e^{-\Lambda b} \leq$ $1 / 2$. For $i+1<j$ notice that $\Psi\left(x_{i+1}\right) \Psi\left(x_{j+1}\right) \leq C$. Then by integrating with respect to $x_{i+1}, x_{j+1}$ we remove the conditional expectation and obtain

$$
A \leq C \int \widehat{\mathbb{E}}_{x_{i}, x_{j}}\left[\left|e^{\lambda W_{\tau_{i}, \tau_{j}}}-1\right|^{n_{i j}} e^{-V_{\tau_{i}}-V_{\tau_{j}}}\right] \Psi\left(x_{i}\right) d x_{i} \Psi\left(x_{j}\right) d x_{j},
$$

where now $\widehat{\mathbb{E}}_{x_{i}, x_{j}}$ denotes expectation over the two pieces $\mathbb{X}_{\tau_{i}}$ and $\mathbb{X}_{\tau_{j}}$ weighted by $\mathcal{W}_{\tau_{i}}^{x_{i}} \otimes \mathcal{W}_{\tau_{j}}^{x_{j}}$, i.e. two independent Wiener measures starting at $x_{i}$ and $x_{j}$, respectively.

Next we estimate the expectation in (5.37),

$$
\begin{aligned}
& \widehat{\mathbb{E}}_{x_{i}, x_{j}} {\left[\left|e^{\lambda W_{\tau_{i}, \tau_{j}}}-1\right|^{n} e^{-V_{\tau_{i}}-V_{\tau_{j}}}\right] \leq \widehat{\mathbb{E}}_{x_{i}, x_{j}}\left[\left|\lambda W_{\tau_{i}, \tau_{j}}\right|^{n} e^{n\left|\lambda W_{\tau_{i}, \tau_{j}}\right|} e^{-V_{\tau_{i}}-V_{\tau_{j}}}\right] } \\
& \leq|\lambda|^{n}\left(\widehat{\mathbb{E}}_{x_{i}, x_{j}}\left[\left|W_{\tau_{i}, \tau_{j}}\right|^{2 n}\right]\right)^{1 / 2}\left(\widehat{\mathbb{E}}_{x_{i}, x_{j}}\left[e^{4 n\left|\lambda W_{\tau_{i}, \tau_{j}}\right|}\right]\right)^{1 / 4}\left(\widehat{\mathbb{E}}_{x_{i}, x_{j}}\left[e^{-4 V_{\tau_{i}}-4 V_{\tau_{j}}}\right]\right)^{1 / 4} .
\end{aligned}
$$

Since $V$ is of Kato-class, we have the uniform bound

$$
\left(\widehat{\mathbb{E}}_{x_{i}, x_{j}}\left[e^{-4 V_{\tau_{i}}-4 V_{\tau_{j}}}\right]\right)^{1 / 4} \leq \sup _{x}\left(\mathbb{E}_{x}\left[e^{\left.-4 V_{[0, b]}\right]}\right)^{1 / 2} \leq C e^{C b},\right.
$$

with some $C>0$. Furthermore, write $W_{\tau_{i}, \tau_{j}}=\int_{\tau_{i}} d X_{t} \int_{\tau_{j}} d X_{s} g_{t s}$ (see (5.41) below) and estimate the double integral below by using the Burkholder-Davis-Gundy inequality,

$$
\begin{aligned}
\widehat{\mathbb{E}}_{x_{i}, x_{j}}\left[\left|W_{\tau_{i}, \tau_{j}}\right|^{2 n}\right] & \leq c_{n} \widehat{\mathbb{E}}_{x_{i}, x_{j}}\left[\left.\left.\left|\int_{\tau_{i}} d t\right| \int_{\tau_{j}} d X_{s} g_{t s}\right|^{2}\right|^{n}\right] \\
& \leq c_{n}\left|\tau_{i}\right|^{n-1} \int_{\tau_{i}} d t \widehat{\mathbb{E}}_{x_{i}, x_{j}}\left[\left|\int_{\tau_{j}} d X_{s} g_{t s}\right|^{2 n}\right] \\
& \leq c_{n}^{2}\left|\tau_{i}\right|^{n-1}\left|\tau_{j}\right|^{n-1} \int_{\tau_{i}} d t \int_{\tau_{j}} d s \widehat{\mathbb{E}}_{x_{i}, x_{j}}\left[\left|g_{t s}\right|^{2 n}\right] \\
& \leq c_{n}^{2} b^{2 n} C^{n}(1+b|i-j-1|)^{-2 n \beta} .
\end{aligned}
$$


Now we estimate also the exponential of the energy. By $e^{|x|} \leq 2 \cosh x$ we get

$$
\widehat{\mathbb{E}}_{x_{i}, x_{j}}\left[e^{4 n\left|\lambda W_{\tau_{i}, \tau_{j}}\right|}\right] \leq \widehat{\mathbb{E}}_{x_{i}, x_{j}}\left[e^{4 n \lambda W_{\tau_{i}, \tau_{j}}}\right]+\widehat{\mathbb{E}}_{x_{i}, x_{j}}\left[e^{-4 n \lambda W_{\tau_{i}, \tau_{j}}}\right] .
$$

Each of the expectations in the right hand side can be similarly estimated by using Lemma 5.9 below:

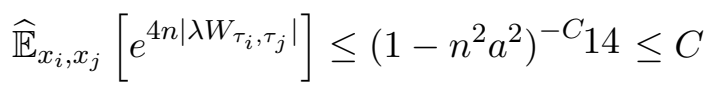

with $a=G_{13} \backslash b(1+b|i-j-1|)^{-\beta}$ and $n a \leq 1 / 2$. Hence, by making use these inequalities (uniform in $x_{i}, x_{j}$ ), and the fact that $\Psi \in L_{1}\left(\mathbb{R}^{d}, d x\right)$, we arrive at

$$
\begin{aligned}
& \left(\int \widehat{\mathbb{E}}_{x_{i}, x_{j}}\left[\left|e^{\lambda W_{\tau_{i}, \tau_{j}}}-1\right|^{n_{i j}} e^{-V_{\tau_{i}}-V_{\tau_{j}}}\right] \Psi\left(x_{i}\right) d x_{i} \Psi\left(x_{j}\right) d x_{j}\right)^{1 / n_{i j}} \\
& \leq C c_{n_{i j}}^{1 / n_{i j}} \lambda b e^{C b}(1+b|i-j-1|)^{-\beta}
\end{aligned}
$$

By using the estimate $c_{k} \leq(2 k)^{2 k}$ for the universal constant in the Burkholder-DavisGundy inequality, we furthermore obtain

$$
c_{n_{i j}}^{1 / n_{i j}}(1+b|i-j-1|)^{-\beta} \leq\left(2 n_{i j}\right)^{2}(1+b|i-j-1|)^{-\beta} .
$$

Recall that $n_{i j}=A|i-j|^{\Delta}$ with $\Delta>1$ and choose $\Delta<\beta$ so that $a n_{i j} \rightarrow 0$ as $|i-j| \rightarrow \infty$ and condition $a n_{i j} \leq 1 / 2$ is satisfied uniformly in $i, j$ for $\lambda$ small enough. Thus there is a constant $q_{10}>0$ such that

$$
\text { r.h.s. ([5.38) } \leq G_{10} \backslash b^{2-2 \Delta} e^{C b}(1+b|i-j-1|)^{2 \Delta-\beta} \leq G_{10} \lambda e^{C b}(1+b|i-j-1|)^{-\delta},
$$

for all $|i-j|>1$ and $b \geq 1$, where $\delta=\beta-2 \Delta>1$ (this is possible by choosing $\Delta>1$ but small enough). For the cases $|i-j|=1$ we can follow a similar strategy to estimate

$$
\left(\mathbb{E}_{\nu_{T}^{\mathbf{x}}}\left[\left|e^{\lambda W_{\tau_{i}, \tau_{j}}}-1\right|^{2 n_{i j}} e^{-V_{\tau_{i}}-V_{\tau_{j}}}\right]\right)^{1 / 2 n_{i j}} \leq q_{11} e^{\mathcal{C}_{12}{ }^{p}},
$$

where the constants do not depend on either $b$ or $n_{i j}$.

\section{$5.4 \quad$ Energy estimates}

Here we estimate $\widehat{\mathbb{E}}_{x, y}\left[e^{\lambda W_{\tau_{i}, \tau_{j}}}\right]$. Below we will prove two lemmas that give the basic estimates by making use of the following result to control exponential integrability of stochastic integrals. The first lemma will be often used for controlling exponential moments of stochastic integrals.

Lemma 5.8 If $X$ is Brownian motion and $f$ is an $\mathcal{F}$-adapted process, we have the bound

$$
\mathbb{E}\left[e^{\int_{0}^{b} f_{s} d X_{s}}\right] \leq\left\{\mathbb{E}\left[e^{2 \int_{0}^{b}\left|f_{s}\right|^{2} d s}\right]\right\}^{1 / 2}
$$


Proof. The proof is a combination of Cauchy-Schwartz and Girsanov Theorems:

$$
\begin{aligned}
\mathbb{E}\left[e^{\int_{0}^{b} f_{s} d X_{s}}\right] & =\mathbb{E}\left[e^{\int_{0}^{b} f_{s} d X_{s}-\int_{0}^{b}\left|f_{s}\right|^{2} d s+\int_{0}^{b}\left|f_{s}\right|^{2} d s}\right] \\
& \leq\left\{\mathbb{E}\left[e^{2 \int_{0}^{b} f_{s} d X_{s}-\frac{1}{2} \int_{0}^{b}\left|2 f_{s}\right|^{2} d s}\right]\right\}^{1 / 2}\left\{\mathbb{E}\left[e^{2 \int_{0}^{b}\left|f_{s}\right|^{2} d s}\right]\right\}^{1 / 2} \\
& \leq\left\{\mathbb{E}\left[e^{2 \int_{0}^{b}\left|f_{s}\right|^{2} d s}\right]\right\}^{1 / 2} .
\end{aligned}
$$

Estimates for separated intervals We turn to estimating exponentials of energy contributions in (5.1) by starting with pairs of intervals that are not adjacent. Let thus $i>j+1$; in this case the exponent has the form $W_{\tau_{i}, \tau_{j}}=\mathcal{J}_{i j}+\mathcal{J}_{j i}$ with

$$
\mathcal{J}_{i, j}=\left\langle C_{\tau_{i}}^{X}, C_{\tau_{j}}^{X}\right\rangle_{W}=\int_{t_{i}}^{t_{i}+b} d X_{t} \int_{t_{j}}^{t_{j}+b} d X_{s} W\left(X_{t}-X_{s}, t-s\right) .
$$

Note that under the measure $\mathcal{W}_{\tau_{i}}^{x_{i}} \otimes \mathcal{W}_{\tau_{j}}^{x_{j}}$ the two currents $\mathbb{X}_{\tau_{i}}$ and $\mathbb{X}_{\tau_{j}}$ are independent and the interaction energy can be written as a double stochastic Itô integral and estimated by using tools borrowed from stochastic analysis.

Lemma 5.9 Let $i>j+1$. There exist positive constants [13, $_{14}$ such that whenever

$$
a:=\lambda C_{13} b\left(1+\left|t_{i}-t_{j}-b\right|\right)^{-\beta}<1,
$$

we have

$$
\widehat{\mathbb{E}}_{x, y}\left[e^{\lambda W_{\tau_{i}, \tau_{j}}}\right] \leq\left[1-a^{2}\right]^{-q} \overline{14} .
$$

Proof. We have

$$
\left(\widehat{\mathbb{E}}_{x, y}\left[e^{\lambda W_{\tau_{i}, \tau_{j}}}\right]\right)^{4} \leq\left(\widehat{\mathbb{E}}_{x, y}\left[e^{2 \lambda \mathcal{J}_{i j}}\right]\right)^{2}\left(\widehat{\mathbb{E}}_{x, y}\left[e^{2 \lambda \mathcal{J}_{j i}}\right]\right)^{2} .
$$

By using (5.40) we obtain

$$
\begin{aligned}
\left(\widehat{\mathbb{E}}_{x, y}\left[e^{\left.\lambda \mathcal{J}_{i j}\right]}\right)^{2}\right. & \leq \widehat{\mathbb{E}}_{x, y}\left[\exp \left(2 \lambda^{2} \int_{t_{i}}^{t_{i}+b} d t\left|\int_{t_{j}}^{t_{j}+b} d X_{s} W\left(X_{t}-X_{s}, t-s\right)\right|^{2}\right)\right] \\
& \leq \int_{t_{i}}^{t_{i}+b} \frac{d t}{b} \widehat{\mathbb{E}}_{x, y}\left[\exp \left(2 b \lambda^{2}\left|\int_{t_{j}}^{t_{j}+b} d X_{s} W\left(X_{t}-X_{s}, t-s\right)\right|^{2}\right)\right] \\
& \leq \int_{t_{i}}^{t_{i}+b} \frac{d t}{b} \widehat{\mathbb{E}}_{x, y} \mathbb{E}_{G}\left[\exp \left(2 \lambda G \sqrt{b} \int_{t_{j}}^{t_{j}+b} d X_{s} W\left(X_{t}-X_{s}, t-s\right)\right)\right]
\end{aligned}
$$

with $G$ a normal random variable defined on a new probability space and $\mathbb{E}_{G}$ the related Gaussian expectation. Then, using again (5.40) yields

$$
\left(\widehat{\mathbb{E}}_{x, y}\left[e^{\left.\lambda W_{\tau_{i}, \tau_{j}}\right]}\right)^{2} \leq \int_{t_{i}}^{t_{i}+b} \frac{d t}{b}\left(\widehat{\mathbb{E}}_{x, y} \mathbb{E}_{G}\left[\exp \left(8 \lambda^{2} G^{2} b \int_{t_{j}}^{t_{j}+b} d s\left|W\left(X_{t}-X_{s}, t-s\right)\right|^{2}\right)\right]\right)^{1 / 2} .\right.
$$


The assumptions on $W$ give

$$
\int_{t_{j}}^{t_{j}+b} d s\left|W\left(Y_{t}-Z_{s}, t-s\right)\right|^{2} \leq \int_{t_{j}}^{t_{j}+b} d s \frac{C}{(1+|t-s|)^{2 \beta}} \leq q_{15} b\left(1+\left|t_{i}-t_{j}-b\right|\right)^{-2 \beta}
$$

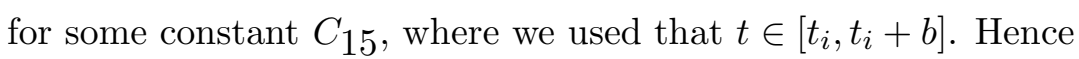

$$
\left(\widehat{\mathbb{E}}_{x, y}\left[e^{2 \lambda \mathcal{J}_{i j}}\right]\right)^{2} \leq\left(\mathbb{E}_{G}\left[\exp \left[32 \lambda^{2} q_{\overline{15}} b^{2}\left(1+\left|t_{i}-t_{j}-b\right|\right)^{-2 \beta} G^{2}\right]\right]\right)^{1 / 2} .
$$

The Gaussian integration can be performed explicitly, yielding

$$
\widehat{\mathbb{E}}_{x, y}\left[e^{2 \lambda \mathcal{J}_{i j}}\right] \leq\left(1-32 \lambda^{2} C_{15} b^{2}\left(1+\left|t_{i}-t_{j}-b\right|\right)^{-2 \beta}\right)^{-d / 8},
$$

as soon as $1-32 \lambda^{2} C_{15} b^{2}\left(1+\left|t_{i}-t_{j}-b\right|\right)^{-2 \beta}>0$. Thus the claim follows.

Estimates for adjacent intervals The interaction energy estimates between adjacent intervals are given by

Lemma 5.10 For all $i=0, \ldots, N-2$ we have $\widehat{\mathbb{E}}_{x, y}\left[e^{\left.\lambda W_{\tau_{i}, \tau_{i+1}}\right]} \leq G_{16 e^{9}}^{9}<\infty\right.$ for sufficiently small $\lambda$.

Proof. By using that $W(x, t)$ is bounded and arguments similar to those of the previous lemma, the required exponential integrability of $W_{\tau_{i}, \tau_{i+1}}$ easily follows, at least for sufficiently small $\lambda$.

\subsection{Properties of the cluster expansion}

We finally show how the convergence of the cluster expansion of $Z_{T}$ and $\log Z_{T}$ seen in Proposition 5.2 imply existence of a limit Gibbs measure $\mu$.

For any subset $A \subset \mathbb{R}$ let

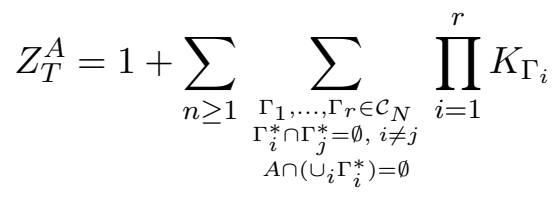

and write $Z_{T}^{\Gamma}:=Z_{T}^{\bar{\Gamma} \cup \Gamma^{*}}$. By the cluster expansion we have

$$
\log Z_{T}^{\Gamma}=1+\sum_{n \geq 1} \sum_{\substack{\Gamma_{1}, \ldots, \Gamma_{r} \in \mathcal{C}_{N} \\ \Gamma^{*} \cap\left(\cup_{i} \Gamma_{i}^{*}\right)=\emptyset}} \phi^{T}\left(\Gamma_{1}, \ldots, \Gamma_{n}\right) \prod_{i=1}^{r} K_{\Gamma_{i}}
$$

Moreover we can define the correlation functions for the clusters by

$$
f_{T}^{\Gamma}=\frac{Z_{T}^{\Gamma}}{Z_{T}}=\exp \left(-\sum_{n \geq 1} \sum_{\substack{\Gamma_{1}, \ldots, \Gamma_{r} \in \mathcal{C}_{N} \\ \Gamma^{*}\left(\cup_{i} \Gamma_{i}^{*}\right) \neq \emptyset}} \phi^{T}\left(\Gamma_{1}, \ldots, \Gamma_{n}\right) \prod_{i=1}^{r} K_{\Gamma_{i}}\right)
$$


and let $f^{\Gamma}=\lim _{T \rightarrow \infty} f_{T}^{\Gamma}$ as the limit exists by the cluster estimates above and general arguments of cluster expansion [40]. Moreover we have the uniform estimate

$$
\left|f_{T}^{\Gamma}\right| \leq 2^{|\bar{\Gamma}|}
$$

for $\lambda$ small enough (the constant 2 can actually be replaced with any number larger than 1 , provided $\lambda$ is chosen correspondingly small). Then existence of the infinite time limit for the measures $\left\{\mu_{T}\right\}_{T}$ follows easily and we have

Proposition 5.11 The local limit $\mu=\lim _{T \rightarrow \infty} \mu_{T}$ exists and satisfies the equality

$$
\mathbb{E}_{\mu}\left[F_{S}\right]=\mathbb{E}_{\chi}\left[F_{S}\right] f^{S}+\sum_{n \geq 1} \sum_{\substack{\Gamma_{1}, \ldots, \Gamma_{r} \in \mathcal{C} \\ \Gamma_{i}^{*} \cap \Gamma_{j}^{*}=\emptyset, i \neq j \\ i: S \cap \Gamma_{i}^{*} \neq \emptyset}} \mathbb{E}_{\chi}\left[F_{S} \prod_{i=1}^{r} \kappa^{\Gamma_{i}}\right] f^{\cup \bar{\Gamma}}
$$

for any bounded, $\mathcal{F}_{S}$-measurable function $F_{S}$, where $S$ is a finite union of intervals of the partition considered in the cluster expansion. Moreover, the measure $\mu$ is invariant with respect to time shift.

Proof. We have

$$
\mathbb{E}_{\mu}\left[F_{S}\right]=\lim _{T \rightarrow \infty} \frac{\int e^{-\lambda W_{T}(X)} F(X) d \nu_{T}(X)}{\int e^{-\lambda W_{T}(X)} d \nu_{T}(X)}=\lim _{T \rightarrow \infty} \frac{Z_{T}(F)}{Z_{T}} .
$$

By the cluster expansion we are led to

$$
Z_{T}(F)=\mathbb{E}_{\chi}\left[F_{S}\right] Z_{T}^{S}+\sum_{n \geq 1} \sum_{\substack{\Gamma_{1}, \ldots, \Gamma_{r} \in \mathcal{C}_{N} \\ \Gamma_{i}^{*} \cap \Gamma_{j}^{*}=\emptyset, i \neq j \\ i: S \cap \Gamma_{i}^{*} \neq \emptyset}} \mathbb{E}_{\chi}\left[F_{S} \prod_{i=1}^{r} \kappa^{\Gamma_{i}}\right] Z_{T}^{\cup \bar{\Gamma}} .
$$

If $F_{S}$ is bounded, standard arguments show that the series on the right hand side is absolutely convergent uniformly in $N$ and thus (5.45) follows. Given the uniqueness of the limiting measure, its invariance with respect to time shifts is a direct consequence of the invariance of the potentials and of the Itô-measure (for more details see [37]).

Corollary 5.12 Let $F \in \mathcal{F}_{[0, b]}$ be a positive random variable. Then $\mathbb{E}_{\mu}[F] \leq C\left(\mathbb{E}_{\nu}\left[F^{2}\right]\right)^{1 / 2}$.

Proof. By using Proposition 5.11 for $S=[0, b]$ (which for fixed $N$ is the interval $\tau_{N / 2}$ of the partition) we have

$$
\mathbb{E}_{\mu}\left[F_{S}\right]=\mathbb{E}_{\chi}\left[F_{S}\right] f^{S}+\sum_{\Gamma_{0}: \bar{\Gamma}_{0} \cap[0, b] \neq \emptyset} \mathbb{E}_{\chi}\left[F_{S} \kappa^{\Gamma_{0}}\right] f^{\Gamma_{0}},
$$

where in the second term the sum is over the only cluster which can overlap with $S$. We have $\left|f^{S}\right| \leq 2,\left|f^{\Gamma_{0}}\right| \leq 2^{\left|\Gamma_{0}^{*}\right|}$. On the other hand, by using Lemma 5.6 and choosing $b$ large enough to ensure that $\sup _{x, y}\left|\pi_{b}(x, y)-1\right| \leq 1 / 2$, we have

$$
\mathbb{E}_{\chi}\left[F_{S}\right]=\int F_{S}(X) \frac{d \nu^{x_{0}, x_{1}}(X)}{\pi_{b}\left(x_{0}, x_{1}\right)} \pi_{b}\left(x_{0}, x_{1}\right) d \omega\left(x_{0}\right) d \omega\left(x_{1}\right) \leq C \mathbb{E}_{\nu}\left[F_{S}\right] .
$$


Furthermore, $\mathbb{E}_{\chi^{N}}\left[\kappa_{a}^{\Gamma_{0}}\right] \leq\left(\mathbb{E}_{\chi^{N}}\left[\xi_{a}\right]\right)^{1 / 2}\left(\mathbb{E}_{\chi^{N}}\left[\left(\kappa^{\Gamma_{0}}\right)^{2}\right]\right)^{1 / 2}$ and by the same arguments as in Proposition 5.3 above we obtain the bound

$$
\sum_{\Gamma_{0}:[0, b] \in \bar{\Gamma}_{0}}\left[\mathbb{E}_{\chi}\left(\kappa^{\Gamma_{0}}\right)^{2}\right]^{1 / 2} 2^{\left|\bar{\Gamma}_{0}\right|} \leq \text { const }
$$

with some constant. Hence we get that $\mathbb{E}_{\mu}\left[F_{S}\right] \leq C \mathbb{E}_{\nu}\left[F_{S}\right]+C\left(\mathbb{E}_{\nu}\left[F_{S}^{2}\right]\right)^{1 / 2}$, which implies the claim.

Theorem 5.13 There exists a unique forward current $\mu^{\sharp}$ on $\Xi$ such that its $\Xi$-marginal is $\mu$. Moreover, under $\mu$ we have $\mathcal{N}_{\alpha}(X)<\infty$ almost surely and the boundary currents are well defined under $\mu^{\sharp}$.

Proof. Corollary 5.12 implies that the measure $\mu$ is absolutely continuous with respect to $\nu$, thus the almost sure events of $\nu$ carry over to $\mu$ and we can consider the lifted measure $\mu^{\sharp}$. This further implies that

$$
\mathbb{E}_{\mu}\left[N_{[k, k+1]}(X)^{3}\right] \leq\left(\mathbb{E}_{\nu}\left[\left(N_{[k, k+1]}(X)\right)^{6}\right]\right)^{1 / 2} \leq C,
$$

independently of $k \in \mathbb{Z}$ and then $\mathbb{E}_{\mu}\left[\mathcal{N}_{\alpha}(X)\right]<\infty$ for any $\alpha>1$. This last condition guarantees the existence of the boundary currents under the measure $\mu^{\sharp}$.

\section{Properties of the Gibbs measure}

\subsection{Dependence on boundary conditions and DLR uniqueness}

Uniqueness in DLR sense means that for any increasing sequence of real numbers $\left\{T_{n}\right\}_{n}$, $T_{n} \uparrow \infty$, and any corresponding sequence of boundary conditions $\left\{Y_{n}\right\}_{n} \subset \Xi$ we have $\mathbb{E}_{\rho_{T_{n}}^{\sharp}\left(\cdot \mid \mathbb{Y}_{n}\right)}\left[F_{B}\right] \rightarrow \mathbb{E}_{\mu}\left[F_{B}\right]$, for every bounded $B \subset \mathbb{R}$, and each bounded and local (i.e., measurable with respect to $\mathcal{F}_{B}$ ) function $F_{B}$ on $\Xi$. However, such a strong statement cannot be made in this context and we have to restrict the class of allowed boundary conditions to be able to control the limit. Fix $\alpha>1$ and let

$$
\Xi_{a}=\left\{Y \in \Xi: \mathcal{N}_{\alpha}(Y) \leq a\right\}, \quad \Xi_{*}=\cup_{a>0} \Xi_{a}
$$

be the set of allowed boundary conditions carrying full $\mu^{\sharp}$ measure. Then we have

Theorem 6.1 For any $a>0$ the measure $\mu$ is unique in DLR sense for any sequence of boundary conditions $\left(\mathbb{Y}_{n}\right)_{n}$ in $\Xi_{a}$, i.e.

$$
\lim _{n \rightarrow \infty} \mathbb{E}_{\rho_{T_{n}}^{\sharp}\left(\cdot \mid \mathbb{Y}_{n}\right)}\left[F_{B}\right]=\mathbb{E}_{\mu}\left[F_{B}\right] .
$$

Proof. We consider the class of bounded local functions $F_{S}$ on $\Xi$ indexed by bounded intervals $S \subset \mathbb{R}$ (that is, $F_{S}$ is measurable with respect to $\mathcal{F}_{S}$ ). It suffices to prove that for any increasing sequence $\left\{T_{n}\right\}, T_{n} \rightarrow \infty$, and any corresponding sequence of boundary conditions $\left(\mathbb{Y}_{n}\right)_{n} \subset \Xi_{a}(\underline{6.2})$ holds for arbitrary $F_{S}$ of the above class. To show this we 
express the conditional expectations appearing above in terms of the cluster representation. We suppose without loss that $S$ consists of a finite union of intervals of the partition of $[-T, T]$.

From now on we follow the steps of the construction of the cluster representation in Section 5.1. Take the same partition of the interval $[-T, T]$ into disjoint segments as before. The interaction energy can then be written as

$$
W_{T}(X \mid Y)=\sum_{0 \leq i<j \leq N} W_{\tau_{i}, \tau_{j}}\left(X_{\tau_{i}}, X_{\tau_{j}}\right)+\sum_{0 \leq i \leq N-1} W_{\tau_{i}, T}^{Y}\left(X_{\tau_{i}}\right)
$$

with the same notations as before, and with

$$
W_{\tau_{i}, T}^{Y}\left(X_{\tau_{i}}\right)=2 \int_{\tau_{i}} w^{C_{T}^{Y+}}\left(X_{t}, t\right) d X_{t}+2 \int_{\tau_{i}} w^{C_{-T}^{Y-}}\left(X_{t}, t\right) d X_{t}
$$

By (4.4) the estimate

$$
\sup _{x \in \mathbb{R}^{d}, t \in \tau}\left|w^{C_{ \pm T}^{Y \pm}}(x, t)\right| \leq \frac{M_{\tau}\left\|C_{ \pm T}^{Y \pm}\right\|_{\mathcal{D}^{\prime}}}{\left(\operatorname{dist}\left(\tau,[-T, T]^{c}\right)+1\right)^{\beta-\alpha}}
$$

easily follows. (Here $[-T, T]^{c}=\mathbb{R} \backslash[-T, T]$ and $\operatorname{dist}\left(\tau_{k},[-T, T]^{c}\right)=\min \{k b,(N-k-1) b\}$.)

Fix the positions $X_{t_{0}}=Y_{-T}^{-}=x_{0}, X_{t_{1}}=x_{1}, \ldots, X_{t_{N-1}}=x_{N-1}, X_{t_{N}}=Y_{T}^{+}=x_{N}$. Similarly to (5.7) introduce the auxiliary measure

$$
d \chi_{N}^{Y}=\prod_{k=0}^{N-1} \frac{e^{-\lambda W_{\tau_{k}, T}^{Y}\left(X_{\tau_{k}}\right)}}{\mathbb{Z}_{\tau_{k}}^{T}\left(Y \mid x_{k}, x_{k+1}\right)} d \nu_{b}^{x_{k}, x_{k+1}}\left(X_{\tau_{k}}\right) \prod_{k=1}^{N-1} d \omega\left(x_{k}\right)
$$

where

$$
\mathbb{Z}_{\tau_{k}}^{T}\left(Y \mid x_{k}, x_{k+1}\right)=\mathbb{E}_{\nu_{b}^{x_{k}, x_{k+1}}}\left[e^{-\lambda W_{\tau_{k}, T}^{Y}\left(X_{\tau_{k}}\right)}\right] .
$$

Also, for every cluster $\Gamma$ consider the function $\kappa_{\Gamma}^{Y}$ defined similarly to (5.6). If $\pm T \notin \Gamma^{*}$, then $\kappa_{\Gamma}^{Y}$ does not depend on $Y$. If $-T \in \Gamma^{*}$ and/or $T \in \Gamma^{*}$, then $\kappa_{\Gamma}^{Y}$ depends on $Y_{-T}^{-}=x_{0}$ and/or $Y_{T}^{+}=x_{N}$, respectively. Next we define the weights

$$
K_{\Gamma}^{Y}=\mathbb{E}_{\chi_{N}^{Y}}\left[\kappa_{\Gamma}^{Y}\right]
$$

in the same manner as in (5.8). The partition function $Z_{T}(Y)$ can be expressed similarly to (5.9) with these altered objects. Note that

(1) for sufficiently small $|\lambda| \neq 0$ the cluster estimate (5.13) obtained in Proposition 5.3 stays essentially valid, i.e.,

$$
\sum_{\substack{\Gamma: \Gamma^{*} \ni 0 \\|\bar{\Gamma}|=n}}\left|K_{\Gamma}^{Y}\right| \leq c \eta^{\prime}(\lambda)^{n}
$$

with $\eta^{\prime}(\lambda)$ going to zero as $\lambda \rightarrow 0$; 
(2) for any fixed $\Gamma$ we have $\lim _{T \rightarrow \infty} \kappa_{\Gamma}^{Y}=\kappa_{\Gamma}$ and

$$
\lim _{T \rightarrow \infty} K_{\Gamma}^{Y}=K_{\Gamma}
$$

both uniformly convergent in $\mathbb{Y} \in \Xi_{a}$.

The proof of these statements goes by the same arguments used in the previous section and it will be omitted. The bound (6.9) can be proven as in Lemma 5.3. Indeed, by using Lemma 5.8 and the bound (6.5) on the influence of the boundary current we have a handle to control the exponential moments of $W_{\tau_{i}, T}^{Y}\left(X_{\tau_{i}}\right)$ in terms of the norm of the boundary current and repeat the proof of Lemma 5.7 to obtain the necessary estimates on cluster activities (with constants depending on $a$ ). A good control of the exponential moments is the key to obtain (6.10).

Then in (6.2) we have

$$
\mathbb{E}_{\mu_{T}}\left[F_{S} \mid Y\right]=\mathbb{E}_{\chi_{T}^{Y}}\left[F_{S}\right] f_{T}^{S}(Y)+\sum_{n \geq 1} \sum_{\substack{\left\{\Gamma_{1}, \ldots, \Gamma_{m}\right\}: \Gamma_{i}^{*} \cap \Gamma_{j}^{*} \neq \emptyset \\ \Gamma_{i}^{*} \cap S \neq 0, \Gamma_{i} \subset[-T, T], i=1, \ldots, m}} \mathbb{E}_{\chi_{T}^{Y}}\left[F_{S} \prod_{i=1}^{m} \kappa_{T}^{Y}\right] f_{T}^{\cup \bar{\Gamma}}(Y)
$$

with the same notations as in (5.47) and $f_{T}^{A}(Y)=Z_{T}^{A}(Y) / Z_{T}(Y)$.

Take now a collection of intervals $\left\{\tau_{i}\right\}=\mathcal{U}$; the partition function $Z_{T}^{\mathcal{U}}(Y):=Z_{T}^{\cup_{\tau_{i} \in \mathcal{U}} \tau_{i}^{*}}(Y)$ can then be written like in (5.9) except for changing $K_{\Gamma}$ for $K_{\Gamma}^{Y}$.

Lemma 6.2 For sufficiently small $|\lambda| \neq 0$ we have the following properties of $f_{T}^{\mathcal{U}}(Y):=$ $Z_{T}^{\mathcal{U}}(Y) / Z_{T}(Y)$. On the one hand,

$$
\left|f_{T}^{\mathcal{U}}(Y)\right| \leq 2^{|\mathcal{U}|}
$$

with $|\mathcal{U}|$ denoting the number of intervals contained in $\mathcal{U}$. On the other hand,

$$
\lim _{T \rightarrow \infty} f_{T}^{\mathcal{U}}(Y)=f^{\mathcal{U}}
$$

uniformly in $\mathbb{Y} \in \Xi_{a}$. Moreover, $f^{\mathcal{U}}$ also satisfies (6.12) above.

Proof. Both statements are direct consequences of the bounds (6.9) and of (6.10) together with the cluster representation of the correlation functions (5.43). By putting

$$
\hat{K}_{\Gamma}^{Y}= \begin{cases}K_{\Gamma}^{Y} & \text { if } \Gamma \text { lies inside }[-T, T] \\ 0 & \text { otherwise }\end{cases}
$$

and using dominated convergence we obtain that $f_{\mathcal{U}}^{Y} \rightarrow f_{\mathcal{U}}$ uniformly as $T \rightarrow \infty$.

We now return to the expression (6.11). By ergodicity of the reference measure

$$
\lim _{T \rightarrow \infty} \mathbb{E}_{\chi_{N}^{Y}}\left[F_{S}\right]=\mathbb{E}_{\chi}\left[F_{S}\right]
$$


and hence the first term of (6.11) converges to $\mathbb{E}_{\chi}\left[F_{S}\right] f_{S}$. By the same argument as above we also obtain

$$
\lim _{N \rightarrow \infty} \mathbb{E}_{\chi_{N}^{Y}}\left[F_{S} \prod_{i=1}^{m} \kappa_{\Gamma_{i}}^{Y}\right]=\mathbb{E}_{\chi}\left[F_{S} \prod_{i=1}^{m} \kappa_{\Gamma_{i}}\right]
$$

uniformly in $Y$, and

$$
\left|\mathbb{E}_{\chi_{N}^{Y}}\left[F_{S} \prod_{i=1}^{m} \kappa_{\Gamma_{i}}^{Y}\right]\right| \leq\left(\sup \left|F_{S}\right|\right) \prod_{i=1}^{m}\left|K_{\Gamma_{i}}^{Y}\right|
$$

which implies

$$
\begin{aligned}
\sum_{\substack{\left\{\Gamma_{1}, \ldots, \Gamma_{m}\right\} \\
\Gamma_{j}^{*} \cap S^{*}=\emptyset, j=1, \ldots, m}}\left(\prod_{i=1}^{m}\left|K_{\Gamma_{i}}^{Y}\right|\right) 2^{\left|\cup_{i} \bar{\Gamma}_{i}\right|} 2^{\left|S^{*}\right|} & \leq 2^{\left|S^{*}\right|} \sum_{m=1}^{\left|S^{*}\right|}\left(\begin{array}{c}
\left|S^{*}\right| \\
m
\end{array}\right)\left(\sum_{\Gamma: \Gamma \ni 0} 2^{|\bar{\Gamma}|}\left|K_{\Gamma}^{Y}\right|\right)^{m} \\
& \leq 2^{\left|S^{*}\right|} \sum_{m=1}^{\left|S^{*}\right|}\left(\begin{array}{c}
\left|S^{*}\right| \\
m
\end{array}\right)\left(\sum_{n=2}^{\infty} 2^{n} c \eta(\lambda)^{n}\right)^{m}<\infty
\end{aligned}
$$

Here $S^{*}$ denotes the time points occurring in $S$. By using now this estimate together with (6.15) and applying Lebesgue's dominated convergence theorem once again, we arrive at

$$
\mathbb{E}_{\chi}\left[F_{S}\right] f_{S}+\sum_{m=1}^{\infty} \sum_{\substack{\left\{\Gamma_{1}, \ldots, \Gamma_{m}\right\} \\ \Gamma_{i}^{*} \cap S^{*} \neq \emptyset, i=1, \ldots, m}} \mathbb{E}_{\chi}\left[F_{S} \prod_{i=1}^{m} \kappa_{\Gamma_{i}}\right] f_{S \cup\left(\cup_{i} \bar{\Gamma}_{i}\right)}=\mathbb{E}_{\mu}\left[F_{S}\right] .
$$

Corollary 6.3 The measure $\mu^{\sharp}$ satisfies the DLR equations

$$
\int_{\Xi} \mathbb{E}_{\rho_{T}^{\sharp}(\cdot \mid \mathbb{Y})}\left[F_{S}\right] d \mu^{\sharp}(\mathbb{Y})=\mathbb{E}_{\mu^{\sharp}}\left[F_{S}\right]
$$

for any $S<T$.

Proof. Note that $\left|\mathbb{E}_{\rho_{T}^{\sharp}(\cdot \mid \mathbb{Y})}\left[F_{S}\right]\right| \leq \sup \left|F_{S}\right|$ and that $\cup_{a>0} \Xi_{a}$ has full $\mu^{\sharp}$-measure so that the left hand side of the equality makes sense. Fix $a>0$. By Theorem 6.1 we have

$$
\int_{\Xi} \mathbb{E}_{\left.\rho_{T}^{\sharp} \cdot \mid \mathbb{Y}\right)}\left[F_{S}\right] d \mu^{\sharp}(\mathbb{Y})=\lim _{T_{2} \rightarrow+\infty} \int_{\Xi} \mathbb{E}_{\rho_{T}^{\sharp}(\cdot \mid \mathbb{Y})}\left[F_{S}\right] d \rho_{T_{2}}^{\sharp}(\mathbb{Y} \mid \mathbb{Z})
$$

uniformly in $Z \in \Xi_{a}$. Then the DLR consistency of the specification implies

$$
\int_{\Xi} \mathbb{E}_{\rho_{T}^{\sharp}(\cdot \mid \mathbb{Y})}\left[F_{S} \mid \mathbb{Y}\right] d \mu^{\sharp}(\mathbb{Y})=\lim _{T_{2} \rightarrow+\infty} \mathbb{E}_{\rho_{T_{2}}^{\sharp}(\cdot \mathbb{Z})}\left[F_{S}\right]=E_{\mu^{\sharp}}\left[F_{S}\right],
$$

proving the statement. 


\subsection{Typical path configurations}

In this section we show that most of $\mu$ 's weight is concentrated on paths that can be characterized by a growth condition. The proof of this depends on a lemma which was already shown in [37, however, we include it here for making the presentation more selfcontained.

Theorem 6.4 Suppose (4.3) holds with $a=b=s$, and $\mu$ is a probability measure obtained by Theorem 5.1 for $V$ and $W$. Then there is a number $C>0$ and a functional $R(X)$, such that

$$
\left|X_{t}\right| \leq(C \log (|t|+1))^{1 /(s+1)}+R(X)
$$

$\mu$-almost surely.

Proof. The strategy of proving this theorem is to derive the typical behaviour of $\mu$ from the typical behaviour of the reference process. This follows through Lemma 6.5 below. Then combining this lemma with Corollary 5.12 gives

$$
\mu\left(\max _{0 \leq t \leq 1}\left|X_{t}\right| \geq a\right) \leq C\left[\mathbb{E}_{\nu}\left(1_{\max _{0 \leq t \leq 1}\left|X_{t}\right| \geq a}\right)\right]^{1 / 2} \leq c^{\prime} e^{-\theta^{\prime} a^{s+1}}
$$

with some constants $c^{\prime}, \theta^{\prime}>0$. Thus under the stationary measure $\mu$

$$
\mu\left(\max _{n \leq t \leq n+1}\left|X_{t}\right| \geq(k \log n)^{1 /(s+1)}\right) \leq \operatorname{const} \frac{1}{n^{k \theta^{\prime}}}
$$

holds. Choosing $k$ so that $k \theta^{\prime}>1$, the Borel-Cantelli Lemma implies that $\mu$-almost surely

$$
|X(t)| \leq(k \log t)^{1 /(s+1)}
$$

for $t \geq T^{*}$, with $T^{*}=T^{*}(X)$ sufficiently large. Writing $R(X)=\max _{|t| \leq T^{*}}|X(t)|$ completes the proof.

Finally we prove the lemma used above.

Lemma 6.5 Let $\nu$ be the measure of the Itô-process for $V$ satisfying (4.3) with exponent $s>2$, and $a>0$. Then there exist $C>0$ and $\theta>0$ such that

$$
\nu\left(\max _{0 \leq t \leq 1}\left|X_{t}\right| \geq a\right) \leq C e^{-\theta a^{s+1}} .
$$

Proof. For the underlying Itô-process we have the Dirichlet operator on $L^{2}\left(\mathbb{R}^{d}, d \nu\right)$

$$
L f=-\Delta f+2(\nabla \log \Psi, \nabla f)
$$

and Dirichlet form

$$
\mathcal{E}(f, f)=-\int f \Delta f d \omega+2 \int f(\nabla \log \Psi, \nabla f) d \omega
$$


with $d \omega=\Psi^{2} d x$, as before. By using Varadhan's Lemma (see Lemma 1.12, [29]), for any $f \in L^{2}(d \omega)$ and every $N>0$

$$
\nu\left(\max _{0 \leq t \leq 1}\left|f\left(X_{t}\right)\right| \geq N\right) \leq \frac{3}{N} \sqrt{\mathcal{E}(f, f)+(f, f)}
$$

holds. Choose $f=f_{a}:=1_{\left\{x \in \mathbb{R}^{d}:|x| \geq a\right\}} * \phi$ by picking a mollifier $\phi$ (with $\|\phi\|_{\infty}<\infty$ ) so that the above convolution is in the domain of $L$. This can be chosen so that the smoothing of the edges of the indicator function takes place in a sphere $S(a)$ of radius a centred at the origin, i.e., with a suitable $\varepsilon>0$ we take $f_{a}(x)=1$ for $x \in \mathbb{R}^{d} \backslash S(a+\varepsilon), f_{a}(x)=0$ for $x \in S(a-\varepsilon)$, and $f_{a}$ is a sufficiently smooth function $\tilde{f}_{a}$ otherwise. Denote these three domains by $D_{1}, D_{2}$ and $D_{3}$, respectively. Setting $N=1$ in (6.23) yields

$$
\nu\left(\max _{0 \leq t \leq 1}\left|X_{t}\right| \geq a\right) \leq 3 \sqrt{\left\|f_{a}\right\|_{L^{2}(d \omega)}^{2}+\left(f_{a}, L f_{a}\right)_{L^{2}(d \omega)}} .
$$

Moreover, we have

$$
\left\|f_{a}\right\|_{L^{2}(d \omega)}^{2}=\int f_{a}^{2}(x) d \omega(x)=\int_{D_{1}} d \omega(x)+\int_{D_{3}} \tilde{f}_{a}^{2}(x) d \omega(x) .
$$

Under the hypothesis on $V$ the standard estimate $\Psi(x) \leq C e^{-\theta|x|^{s+1}}$ holds by Carmona's results [7] for the ground state $\Psi$, with some $C, \theta>0$. This bound further leads to

$$
\int_{D_{1}} d \omega(x) \leq c e^{-\theta a^{s+1}}
$$

where $c, \theta>0$ are independent of $a$. A similarly estimate is valid for $D_{3}$. On the other hand, since $\tilde{f}_{a}$ is smooth enough and $\max _{D_{3}}\left\{\left|\nabla \tilde{f}_{a}\right|,\left|\Delta \tilde{f}_{a}\right|, \Delta \tilde{f}_{a}^{2}\right\} \leq m<\infty$, we get

$$
\left(\tilde{f}_{a}, L \tilde{f}_{a}\right) \leq c^{\prime} e^{-\theta a^{s+1}}
$$

with suitable $c^{\prime}>0$. A similar estimate is obtained also for the remaining two domains.

\subsection{Mixing properties}

Since $\mu$ is constructed in a way that offers no immediate access to computations with this measure, it is important to derive further basic information on $\mu$ by using the cluster expansion. We give here one last result of this paper.

Theorem 6.6 Let $F, G$ be two bounded functions, the first measurable with respect to $\mathcal{F}_{I}$, the second with respect to $\mathcal{F}_{J}$, where $I, J$ are distinct intervals of the partition considered in the cluster expansion above. Then the estimate on the covariance

$$
\begin{aligned}
& \operatorname{cov}_{\mu}(F ; G)=\mathbb{E}_{\mu}[F G]-\mathbb{E}_{\mu}[F] \mathbb{E}_{\mu}[G] \\
& \left|\operatorname{cov}_{\mu}(F ; G)\right| \leq \mathrm{const} \frac{\sup |F| \sup |G|}{|t-s|^{\vartheta}+1}
\end{aligned}
$$

holds, where $\vartheta>0$ and the constant prefactor is independent of $F, G$. 
Proof. First recall formula (5.45) which applied to $F$ (and similarly to $G$ ) gives

$$
\mathbb{E}_{\mu}[F]=\mathbb{E}_{\nu}[F] f^{I}+\sum_{\Gamma_{0}: I \cap \Gamma_{0}^{*} \neq \emptyset} K^{\Gamma_{0}}(F) f^{\Gamma_{0}^{*}},
$$

where we let $K_{\Gamma_{0}}(F)=\mathbb{E}_{\chi}\left[F \kappa_{\Gamma_{0}}\right]$. Furthermore, consider $f^{A}$ estimated as before like $\left|f^{A}\right| \leq 2^{|A|}$. For $A_{1} \cap A_{2}=\emptyset$ we have

$$
\left|f^{A_{1} \cup A_{2}}-f^{A_{1}} f^{A_{2}}\right| \leq \mathrm{const} \frac{2^{\left|A_{1}\right|+\left|A_{2}\right|}}{\operatorname{dist}\left(A_{1}, A_{2}\right)^{\zeta}},
$$

with some $\zeta>0$. This estimate can easily be obtained by the general results in [40. Now we write

$$
\begin{aligned}
\mathbb{E}_{\mu}[F G]=\mathbb{E}_{\nu}[F] \mathbb{E}_{\nu}[G] f^{I \cup J} & +\sum_{\substack{\Gamma_{1} \\
\Gamma_{1}^{*} \cap I \neq \emptyset, \Gamma_{1}^{*} \cap J=\emptyset}} \mathbb{E}_{\chi}\left[F \kappa_{\Gamma_{1}}\right] \mathbb{E}_{\chi}[G] f^{J \cup \Gamma_{1}^{*}}+\sum_{\substack{\Gamma_{2} \\
\Gamma_{2}^{*} \cap J \neq \emptyset, \Gamma_{2}^{*} \cap I=\emptyset}} \mathbb{E}_{\chi}\left[G \kappa_{\Gamma_{2}}\right] \mathbb{E}_{\chi}[F] f^{I \cup \Gamma_{2}^{*}} \\
& +\sum_{\substack{\Gamma_{1}, \Gamma_{2}: \Gamma_{1}^{*} \cap \Gamma_{2}^{*}=\emptyset \\
\Gamma_{2}^{*} \cap J \neq \emptyset, \Gamma_{2}^{*} \cap I \neq \emptyset}} \mathbb{E}_{\chi}\left[F \kappa_{\Gamma_{1}}\right] \mathbb{E}_{\chi}\left[G \kappa_{\Gamma_{2}}\right] f_{1}^{\Gamma_{1}^{*} \cup \Gamma_{2}^{*}} \sum_{\substack{\Gamma \\
\Gamma^{*} \cap J \neq \emptyset, \Gamma * \cap I \neq \emptyset}} \mathbb{E}_{\chi}\left[F G \kappa_{\Gamma}\right] f^{\Gamma^{*}} .
\end{aligned}
$$

From here and (6.25) we obtain

$$
\begin{aligned}
& \operatorname{cov}_{\mu}(F ; G)=\mathbb{E}_{\chi}[F] \mathbb{E}_{\chi}[G]\left(f^{I \cup J}-f^{I} f^{J}\right) \\
& +\sum_{\substack{\Gamma_{1} \\
\Gamma_{1}^{*} \cap\left\lceil\neq \emptyset, \Gamma_{1}^{*} \cap J=\emptyset\right.}} \mathbb{E}_{\chi}\left[F \kappa_{\Gamma_{1}}\right] \mathbb{E}_{\chi}[G]\left(f^{J \cup \Gamma_{1}^{*}}-f^{J} f^{\Gamma_{1}^{*}}\right) \\
& +\sum_{\substack{\Gamma_{2} \\
\Gamma_{2}^{*} \cap J \neq \emptyset, \Gamma_{2}^{*} \cap I=\emptyset}} \mathbb{E}_{\chi}\left[G \kappa_{\Gamma_{2}}\right] \mathbb{E}_{\chi}[F]\left(f^{I \cup \Gamma_{2}^{*}}-f^{I} f^{\Gamma_{2}^{*}}\right) \\
& +\sum_{\substack{\Gamma_{1}, \Gamma_{2}: \Gamma_{1}^{*} \cap \Gamma_{2}^{*}=\emptyset \\
\Gamma_{2}^{*} \cap J \neq \emptyset, \Gamma_{2}^{*} \cap I \neq \emptyset}} \mathbb{E}_{\chi}\left[F \kappa_{\Gamma_{1}}\right] \mathbb{E}_{\chi}\left[G \kappa_{\Gamma_{2}}\right]\left(f^{\Gamma_{1}^{*} \cup \Gamma_{2}^{*}}-f^{\Gamma_{1}^{*}} f^{\Gamma_{2}^{*}}\right) \\
& +\sum_{\substack{\Gamma \\
\Gamma^{*} \cap J \neq \emptyset, \Gamma * \cap \mp \neq \emptyset}} \mathbb{E}_{\chi}\left[F G \kappa_{\Gamma}\right] f^{\Gamma^{*}} \\
& \text { - } \sum_{\substack{\Gamma \\
\Gamma^{*} \cap J \neq \emptyset \Gamma^{*} \cap I \neq \emptyset}} \mathbb{E}_{\chi}[F] \mathbb{E}_{\chi}\left[G \kappa_{\Gamma}\right] f^{\Gamma^{*}} f^{I} f^{\Gamma^{*}} \\
& -\sum_{\substack{\Gamma \\
\Gamma^{*} \cap J \neq \emptyset, \Gamma^{*} \cap I \neq \emptyset}} \mathbb{E}_{\chi}\left[F \kappa_{\Gamma}\right] \mathbb{E}_{\chi}[G] f^{\Gamma^{*}} f^{J} f^{\Gamma^{*}} \\
& -\sum_{\substack{\Gamma_{1}, \Gamma_{2}: \Gamma_{1}^{*} \cap \Gamma_{2}^{*} \neq \emptyset \\
\Gamma_{2}^{*} \cap J \neq \emptyset, \Gamma_{2}^{*} \cap I \neq \emptyset}} \mathbb{E}_{\chi}\left[F \kappa_{\Gamma_{1}}\right] \mathbb{E}_{\chi}\left[G \kappa_{\Gamma_{2}}\right] f^{\Gamma_{1}^{*}} f^{\Gamma_{2}^{*}}
\end{aligned}
$$


For estimating the first four terms at the right hand side above we use (6.26) along with the bound

$$
\left|\mathbb{E}_{\mathcal{P}}\left[F \kappa_{\Gamma}\right]\right| \leq \frac{\sup |F|}{\left(\operatorname{diam} \Gamma^{*}\right)^{\zeta^{\prime}}+1} E_{\Gamma}\left(\delta^{\prime}, \varepsilon^{\prime}\right)
$$

$i=1,2$, where $E_{\Gamma}\left(\delta^{\prime}, \varepsilon^{\prime}\right)$ is the function appearing at the right hand side of estimate (5.32) with slightly modified entries $\left(\delta^{\prime}, \varepsilon^{\prime}\right.$ instead of $\left.\delta, \varepsilon ; \delta^{\prime}>1\right)$ so that (5.13) still holds. Here $\zeta^{\prime}=\delta-\delta^{\prime}>0$, and we used in addition that

$$
\frac{1}{\left(\operatorname{diam} \Gamma^{*}\right)^{\zeta^{\prime}}+1} \frac{1}{\operatorname{dist}\left(I, \Gamma^{*}\right)^{\zeta}+1} \leq \frac{1}{\operatorname{dist}(I, J)^{\vartheta}+1}
$$

whenever $J \cap \Gamma^{*} \neq \emptyset$ (similarly for $I$ ), and

$$
\frac{1}{\left(\operatorname{diam} \Gamma_{1}^{*}\right)^{\zeta^{\prime}}+1} \frac{1}{\left(\operatorname{diam} \Gamma_{2}^{*}\right)^{\zeta^{\prime}}+1} \frac{1}{\operatorname{dist}\left(\Gamma_{1}^{*}, \Gamma_{2}^{*}\right)^{\zeta}} \leq \frac{1}{\operatorname{dist}(I, J)^{\vartheta}+1}
$$

for $I \cap \Gamma_{1}^{*} \neq \emptyset, J \cap \Gamma_{2}^{*} \neq \emptyset$, and $\vartheta=\min \left\{\zeta, \zeta^{\prime}\right\}>0$.

Next, in the fifth term above we use that $\operatorname{diam} \Gamma^{*} \geq \operatorname{dist}(I, J)$ whenever $I \cap \Gamma^{*} \neq \emptyset$, $J \cap \Gamma^{*} \neq \emptyset$, and that

$$
\left|\mathbb{E}_{\chi}\left[F G \kappa_{\Gamma}\right]\right| \leq \sup |F| \sup |G| \frac{E_{\Gamma}\left(\delta^{\prime}, \varepsilon^{\prime}\right)}{\left(\operatorname{diam} \Gamma^{*}\right)^{\prime}+1} .
$$

For the remaining three terms in the sum above we apply the same argument. Thus for the full sum the corresponding bounds become

$$
\text { const } \frac{\sup |F| \sup |G|}{\operatorname{dist}(I, J)^{\vartheta}+1} E_{\Gamma_{1}}\left(\delta^{\prime}, \varepsilon^{\prime}\right) E_{\Gamma_{2}}\left(\delta^{\prime}, \varepsilon^{\prime}\right) 2^{\left|\Gamma_{1}^{*}\right|+\left|\Gamma_{2}^{*}\right|},
$$

whenever $I \cap \Gamma_{1}^{*} \neq \emptyset, J \cap \Gamma_{2}^{*} \neq \emptyset$, respectively

$$
\text { const } \frac{\sup |F| \sup |G|}{\operatorname{dist}(I, J)^{\vartheta}+1} E_{\Gamma}\left(\delta^{\prime}, \varepsilon^{\prime}\right) 2^{\left|\Gamma^{*}\right|}
$$

in the other cases. Then using Proposition 5.3 we can prove boundedness of the sums over $\Gamma_{1}, \Gamma_{2}$ or $\Gamma$, concluding the proof.

\section{References}

[1] Bessaih, H., Gubinelli, M. and Russo, F.: The evolution of a random vortex filament, Ann. Probab. 33 (2005), 1825-1855

[2] Betz, V.: Existence of Gibbs measures relative to Brownian motion, Markov Proc. Rel. Fields 9 (2003), 85-102

[3] Betz, V., Hiroshima, F., Lőrinczi, J., Minlos, R.A. and Spohn, H.: Ground state properties of the Nelson Hamiltonian - A Gibbs measure-based approach, Rev. Math. Phys. 14 (2002), 173-198 
[4] Betz, V. and Lörinczi, J.: Uniqueness of Gibbs measure relative to Brownian motion, Ann. IHP, Probab. Es Stat. 39 (2003), 877-889

[5] Bolthausen, E.: On the construction of the three-dimensional polymer measure, Probab. Theory Related Fields 97 (1993), 81-101

[6] Bovier, A., Felder, G. and Fröhlich, J.: On the critical properties of the Edwards and the self-avoiding walk model of polymer chains, Nuclear Phys. B230 (1984), 119-147

[7] Carmona, R.: Pointwise bounds for Schrödinger eigenstates, Commun. Math. Phys. 62 (1978), 97-106

[8] Coutin, L. and Lejay, A.: Semi-martingales and rough paths theory, Electron. J. Probab. 10 (2005), 761-785

[9] Coutin, L. and Qian, Z.: Stochastic analysis, rough path analysis and fractional Brownian motions, Probab. Theory Rel. Fields 122 (2002), 108-140

[10] De Angelis, G.F., Jona-Lasinio, G. and Sirugue, M.: Probabilistic solution of Pauli type equations, J. Phys. A 16 (1983), 2433-2444

[11] De Angelis, G.F. and Jona-Lasinio, G.: A stochastic description of a spin-1/2 particle in a magnetic field, J. Phys. A15 (1982), 2053-2061

[12] Dobrushin, R.L.: Analyticity of correlation functions in one-dimensional classical systems with slowly decreasing potentials, Commun. Math. Phys. 32 (1973), 269-289

[13] Dobrushin, R.L.: Analyticity of correlation functions for one-dimensional classical systems with power-law decay of the potential, Math. USSR Sbornik 23 (1973), 13-44

[14] Donsker, M.D. and Varadhan, S.R.S.: Asymptotics for the polaron, Commun. Pure Appl. Math. 36 (1983), 505-528

[15] Feyel, D. and de La Pradelle, A.: Curvilinear integrals along enriched paths, Electron. J. Probab. 11 (2006), 860-892

[16] Flandoli, F.: On a probabilistic description of small scale structures in 3D fluids, Ann. IHP, Probab. \& Stat. 38 (2002), 207-228

[17] Flandoli, F., Giaquinta, G., Gubinelli, M. and Tortorelli, V.M.: Stochastic currents, Stoch. Proc. Appl. 155 (2005), 1583-1601

[18] Flandoli, F. and Gubinelli, M.: The Gibbs ensemble of a vortex filament, Probab. Theory Rel. Fields 122 (2002), 317-340

[19] Flandoli, F. and Gubinelli, M.: Random Currents and Probabilistic Models of Vortex Filaments, Seminar on Stochastic Analysis, Random Fields and Applications IV, Ascona 2002, Progress in Probabilty 58, Birkhäuser Verlag, Basel, 2004 
[20] F. Flandoli and M. Gubinelli. Statistics of a vortex filament model, Electr. Jour. Prob. 10 (2005), 865-900

[21] F. Flandoli, M. Gubinelli, and F. Russo. On the regularity of stochastic currents, fractional Brownian motion and applications to a turbulence model, preprint (2007)

[22] Georgii, H.-O.: Gibbs Measures and Phase Transitions, Berlin, New York: de Gruyter, 1988

[23] Gubinelli, M.: Controlling rough paths, J. Funct. Anal. 216 (2004), 86-140

[24] Gubinelli, M. and Lőrinczi, J.: Ultraviolet renormalization of Nelson's Hamiltonian through functional integration, preprint (2007)

[25] Hariya, Y. and Osada, H.: Diffusion processes on path spaces with interactions, Rev. Math. Phys. 13 (2001), 199-220

[26] Hiroshima, F.: Functional integral representations of quantum electrodynamics, Rev. Math. Phys. 9 (1997), 489-530

[27] Hiroshima, F. and Lőrinczi, J.: Functional integral representation of a non-relativistic quantum particle with spin $1 / 2$, preprint (2007)

[28] Hiroshima, F. and Lörinczi, J.: Localization of the ground state in the Pauli-Fierz model at weak couplings, preprint (2007)

[29] Kipnis, C. and Varadhan, S.R.S.: Central limit theorem for additive functionals of reversible Markov processes and applications to simple exclusions, Commun. Math. Phys. 104 (1986), 1-19

[30] Le Gall, J.F.: Temps locaux d'intersection renormalisés et développement asymptotique du volume de la saucisse de Wiener plane, C. R. Acad. Sci. Paris Sér. I Math. 304 (1987), 339-342

[31] Le Gall, J.F.: Exponential moments for the renormalized self-intersection local time of planar Brownian motion, In Séminaire de Probabilités, XXVIII, LNM 1583, Springer, Berlin, 1994, pp. 172-180

[32] Lejay, A.: An introduction to rough paths, in: Séminaire de Probabilités XXXVII, Lecture Notes in Math. 1832, Springer, Berlin, 2003, pp. 1-59

[33] Lyons, T.: Differential equations driven by rough signals, Rev. Mat. Iberoamericana 14 (1998), 215-310

[34] Lyons, T. and Qian, Z.: System Control and Rough Paths, Oxford University Press, 2002

[35] Lörinczi, J.: Gibbs measures on Brownian paths, in In and Out of Equilibrium: Physics with a Probability Flavour, V. Sidoravicius, ed., Birkhäuser, 2002, pp. 349-362 
[36] Lörinczi, J. and Mauser, N.J.: A stochastic approach to the bipolaron model, RIMS S. Kokyuroku, 1482 (2006), 1-10

[37] Lőrinczi, J. and Minlos, R.A.: Gibbs measures for Brownian paths under the effect of an external and a small pair potential, J. Stat. Phys. 105 (2001), 605-647

[38] Lörinczi, J., Minlos, R.A. and Spohn, H.: The infrared behaviour in Nelson's model of a quantum particle coupled to a massless scalar field, Ann. Henri Poincaré 3 (2002), $1-28$

[39] Lörinczi, J., Minlos, R.A. and Spohn, H.: Infrared regular representation of the three dimensional massless Nelson model, Lett. Math. Phys. 59 (2002), 189-198

[40] Malyshev, V.A. and Minlos, R.A.: Gibbs Random Fields, Kluwer Academic Publishers, 1991

[41] Nelson, E.: Interaction of nonrelativistic particles with a quantized scalar field, $J$. Math. Phys. 5 (1964), 1990-1997

[42] Nualart, D., Rovira, C. and Tindel, S.: Probabilistic models for vortex filaments based on fractional Brownian motion, RACSAM Rev. R. Acad. Cienc. Exactas Fís. Nat. Ser. A Mat. 95 (2001), 213-218

[43] Osada, H. and Spohn, H.: Gibbs measures relative to Brownian motion, Ann. Probab. 27 (1999), 1183-1207

[44] Revuz, D. and Yor, M.: Continuous martingales and Brownian motion, Springer, Berlin, 1999

[45] Spohn, H.: The polaron at large total momentum, J. Phys. A 21 (1988), 1199-1211.

[46] Simon, B.: Functional Integration and Quantum Mechanics, Academic Press, 1975 (2nd edition, 2005)

[47] Simon, B.: Schrödinger operators, Bull. AMS 7 (1982), 447-526

[48] Symanzik, K.: Euclidean quantum field theory, in: Local Quantum Theory, R. Jost (ed.), New York, Academic Press, 1969

[49] Westwater, J.: On Edwards' model for polymer chains, in: Trends and Developments in the Eighties (Bielefeld, 1982/1983), World Sci. Publishing, Singapore, 1985, pp. 384-404

[50] Westwater, J.: On Edwards' model for polymer chains. II. The self-consistent potential, Comm. Math. Phys. 79 (1981), 53-73

[51] Westwater, J.: On Edwards' model for polymer chains III. Borel summability, Comm. Math. Phys. 84 (1982), 459-470 
[52] Westwater, J.: On Edwards' model for long polymer chains, Comm. Math. Phys. 72 (1980), 131-174

[53] Yor, M.: Précisions sur l'existence et la continuité des temps locaux d'intersection du mouvement brownien dans $\mathbf{R}^{2}$, in: Séminaire de Probabilités, XX, 1984/85, LNM 1204, Springer, Berlin, 1986, pp. 532-542

[54] Young, L.C.: An inequality of Hölder type connected with Stieltjes integration, Acta Math. 67 (1936), 251-282 